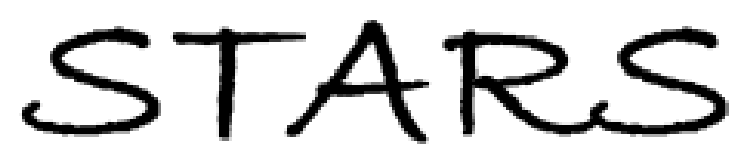

University of Central Florida

STARS

Retrospective Theses and Dissertations

1985

\title{
Computerized Displacement Data Analysis in Laser Speckle Photography
}

\author{
Bijan Chitsaz \\ University of Central Florida
}

Part of the Engineering Commons

Find similar works at: https://stars.library.ucf.edu/rtd

University of Central Florida Libraries http://library.ucf.edu

This Masters Thesis (Open Access) is brought to you for free and open access by STARS. It has been accepted for inclusion in Retrospective Theses and Dissertations by an authorized administrator of STARS. For more information, please contact STARS@ucf.edu.

\section{STARS Citation}

Chitsaz, Bijan, "Computerized Displacement Data Analysis in Laser Speckle Photography" (1985).

Retrospective Theses and Dissertations. 4818.

https://stars.library.ucf.edu/rtd/4818

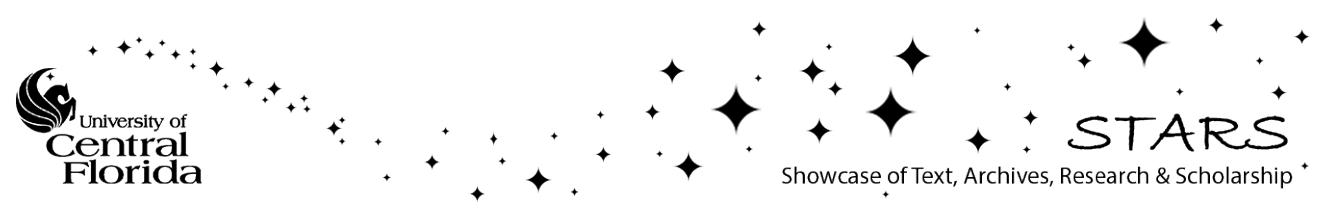




$$
\begin{aligned}
& \text { COMPUTERIZED DISPLACEMENT DATA } \\
& \text { ANALYSIS IN LASER SPECKLE PHOTOGRAPHY }
\end{aligned}
$$

BY

BIJAN CHITSAZ

B.S.E., University of Central Florida, 1983

\section{RESEARCH REPORT}

Submitted in partial fulfillment of the requirements for the degree of Master of Science

in the Graduate Studies Program of the College of Engineering University of Central Florida

Orlando, Florida

Fal1 Term

1985 
ABSTRACT

Laser speckle photography is an experimental technique which provides a non-contact means for direct measurement of in-plane displacements of objects subjected to different loading conditions. It requires a camera system to record two images of the object that are illuminated with laser light on a high resolution film. The double-exposure film (specklegram) is a permanent record of the displacement information of the object. Point-by-point analysis of the specklegram will yield the displacement data, but requires considerable time to complete the data reduction.

An automated data analysis system is developed and described in this report. He-Ne laser and computer-controlled translation stages holding the specklegram represent the first part of the system. A linear diode sensor is used to digitize the generated fringe pattern. Finally, a microcomputer and a spectrum analyzer are used to perform the analysis. The system has been successfully applied to the computation of displacements of a cantilever beam. Comparisons to theoretical results indicate that the system is indeed a viable one and may be used for more complex displacement analysis. 
LIST OF TABLES . . . . . . . . . . . . . . . . . . . . . iv

LIST OF FIGURES . . . . . . . . . . . . . . . . . . . . . . . v v

Chapter

I. INTRODUCTION . . . . . . . . . . . . . . . . . . . . 1

Photoelasticity Method . . . . . . . . . . 1

Moire Method . . . . . . . . . . . . . . 3

Holographic Interferometry Method . . . . . . . 5

Electrical-Resistance Strain Gage Method . . . . 9

Speckle Photography Method . . . . . . . . 10

II. LITERATURE REVIEW . . . . . . . . . . . . . . . 16

III. THEORY OF FRINGE FORMATION . . . . . . . . . . . . . . . 21

IV. COMPUTER-AIDED DATA ACQUISITION SYSTEM HARDWARE

AND EXPERIMENTAL SET-UP . . . . . . . . . . . . . . . 32

Laser . . . . . . . . . . . . . . . . 32

Translation Stages . . . . . . . . . . . . 35

CCD-113D Photodiode Sensor . . . . . . . . . 35

Oscilloscope . . . . . . . . . . . . . . 35

Spectrum Analyzer . . . . . . . . . . . . 37

HP-85 Microcomputer . . . . . . . . . . . 37

V. RESULTS AND DISCUSSION . . . . . . . . . . . . . . 38

VI. CONCLUSION AND RECOMMENDATIONS . . . . . . . . . . . 76

Appendices

A. OPERATION OF CC-1 CONTROLlable tRANSLATiON STAGES • • 79

B. INSTRUCTIONS ON CCD-133 LINE SCANNER . . . . . . . . . 83

C. DATA MANIPULATION PROGRAMS . . . . . . . . . . . . . 91

REFERENCES . . . . . . . . . . . . . . . . . . 116 


\section{LIST OF TABLES}

1. Fringe Spacing Versus Inverse of Frequency . . . . . . . 45

2. Fringe Number Versus Frequency . . . . . . . . . . . . . . 51

3. Experimental Versus Theoretical Displacement Values at Different Locations Along Centerline of the Beam . • . 73 


\section{LIST OF FIGURES}

1. (a) Fringe Formation in a Model Under Stress and (b) a Circular Polariscope . . . . . . . . . . . . . . 2

2. (a) $M$ and S Arrays, (b) $M$ and S Arrays Set Beside Each Other and (c) Relative Displacement Between M and S Arrays . . . . . . . . . . . . . . . . . . 4

3. Off-axis Holography: (a) System for Recording the Hologram, (b) Reconstruction of the True, Virtual Image and (c) Reconstruction of the Conjugate, Real Image . . . . . . . . . . . . . . . . . . 7

4. Normal Deformation of a Cantilever Beam: (a) Side View of the Beam (Displacement is in the $+z$ Direction and Illumination in the $-z$ Direction) and (b) Fringe Pattern Observed on the Front of the Beam . . . . . . . 8

5. Configuration of Metal-Foil Strain Gages . . . . . . . . 11

6. Two-Exposure Speckle Photography for Measurement of In-Plane Deformation: (a) Recording System, (b) Set-Up for Fringe Formation and (c) Formation of Fringe in the Back Focal Plane . . . . . . . . . . . . . . . 12

7. Diagram of Fringe Orientation and the Respective $x$ and y Components of Fringe Spacing . . . . . . . . . . . . 14

8. Illustration of Young's Experiment . . . . . . . . . . . 22

9. Origin of Light and Dark Bands in an Interference Pattern . . . . . . . . . . . . . . . . . 23

10. Theoretical Interpretation of the Double-Slit Experiment . 24

11. Photograph of an Interference Pattern in Young's Experiment . . . . . . . . . . . . . . . . . 28

12. Fringe Pattern Formation by Diffraction at a DoubleExposure Speckle Photograph . . . . . . . . . . . .

13. Time Record and FFT Plots of Sinewave Having Frequency of $400 \mathrm{~Hz}$. . . . . . . . . . . . . . . . . . 
14. Time Record and FFT Plots of Sinewave Having Frequency of $800 \mathrm{~Hz}$. . . . . . . . . . . . . . . . 31

15. Block Diagram of the Experimental Set-Up . . . . . . . . 33

16. Photograph of the Actual Experimental Set-Up . . . . . . 34

17. Photograph of CCD-133D, Photodiode Sensor . . . . . . 36

18. Artificial Fringes and Their Respective Spacing Used in the Experiment . . . . . . . . . . . . . . . 39

19. Artificial Fringe Analysis Experimental Set-Up . . . . . . 40

20. Time Record and FFT Plots of an Artificial Fringe with 0.03 in Spacing . . . . . . . . . . . . . . 41

21. Time Record and FFT Plots of an Artificial Fringe with 0.042 in Spacing................. . . 41

22. Time Record and FFT Plots of an Artificial Fringe with 0.05 in Spacing . . . . . . . . . . . . . . 42

23. Time Record and FFT Plots of an Artificial Fringe with 0.058 in Spacing.................. 42

24. Time Record and FFT Plots of an Artificial Fringe with 0.075 in Spacing. . . . . . . . . . . . . . .

25. Time Record and FFT Plots of an Artificial Fringe with 0.100 in Spacing. . . . . . . . . . . . . . 43

26. Plot of Fringe Spacing Versus Inverse of Frequency . . . 44

27. Experimental Set-Up for Producing Double-Exposure Specklegram of the Cantilever Beam Experiment . . . . . . 46

28. Time Record and FFT Plots of 3-Fringe Case... . . . . 48

29. Time Record and FFT Plots of 4-Fringe Case... . . . 48

30. Time Record and FFT Plots of 5-Fringe Case . . . . . . 49

31. Time Record and FFT Plots of 6-Fringe Case . . . . . . 49

32. Time Record and FFT Plots of 7-Fringe Case . . . . . . 50

33. Plot of Fringe Number Versus Frequency . . . . . . . . 52 
34. Scan Locations on the Fringe Halo . . . . . . . . . . . 53

35. Time Record and FFT Plots of First Scan to the Right of Bright Spot . . . . . . . . . . . . . . . . 54

36. Time Record and FFT Plots of Second Scan to the Right of Bright Spot . . . . . . . . . . . . . . . . . . . . 54

37. Time Record and FFT Plots of Third Scan to the Right of Bright Spot . . . . . . . . . . . . . . . . . 55

38. Time Record and FFT Plots of Fourth Scan to the Right of Bright Spot . . . . . . . . . . . . . . . . . 55

39. Time Record and FFT Plots of First Scan to the Left of Bright Spot . . . . . . . . . . . . . . 56

40. Time Record and FFT Plots of Second Scan to the Left of Bright Spot . . . . . . . . . . . . . . 56

41. Time Record and FFT Plots of Third Scan to the Left of Bright Spot . . . . . . . . . . . . . . . .

42. Time Record and FFT Plots of Fourth Scan to the Left of Bright Spot . . . . . . . . . . . . . . 57

43. Average of Eight $\mathrm{FFT}^{2}$ Plots... . . . . . . . . . . . 59

44. Diagram of Cantilever Beam and Locations of Various Sample Points... . . . . . . . . . . . . . . . . . 60

45. Experimental Set-Up for the Analysis of the Displacements of the Cantilever Beam . . . . . . . . . 61

46. Time Record and FFT Plots of Point 1 . . . . . . . . . . 62

47. Time Record and FFT Plots of Point 2 . . . . . . . . . . 62

48. Time Record and FFT Plots of Point 3 . . . . . . . . . . 63

49. Time Record and FFT Plots of Point 4 . . . . . . . . . . 63

50. Time Record and FFT Plots of Point 5 . . . . . . . . . . 64

51. Time Record and FFT Plots of Point 6 . . . . . . . . . . 64

52. Time Record and FFT Plots of Point 7 . . . . . . . . 65

53. Time Record and FFT Plots of Point 8 . . . . . . . . 65 
54. Time Record and FFT Plots of Point 9 . . . . . . . . . . 66

55. Time Record and FFT Plots of Point 10 . . . . . . . . 66

56. Time Record and FFT Plots of Point 11 . . . . . . . . 67

57. Time Record and FFT Plots of Point 12 . . . . . . . . . 67

58. Time Record and FFT Plots of Point 13......... . . 68

59. Time Record and FFT Plots of Point 14 . . . . . . . . 68

60. Time Record and FFT Plots of Point 15 . . . . . . . . 69

61. Time Record and FFT Plots of Point 16 . . . . . . . . . 69

62. Time Record and FFT Plots of Point 17 . . . . . . . . 70

63. Time Record and FFT Plots of Point 18 . . . . . . . . 70

64. Time Record and FFT Plots of Point 19... . . . . . . 71

65. Time Record and FFT Plots of Point 20 . . . . . . . . . 71

66. Plot of Vertical Displacement Versus Beam Length . . . 74

67. Block Diagram of CCD-133D . . . . . . . . . . . . 84

68. Timing Diagram of CCD-133D . . . . . . . . . . . . 85

69. CCD-133D Characteristic Charts . . . . . . . . . . 88

70. Equipment and Their Respective Adjustments . . . . . . 89

71. Schematic Diagram of Set-Up . . . . . . . . . . . . 90 


\section{CHAPTER I}

\section{INTRODUCTION}

The demands for greater optimization in the design of load resisting structures have created a need for better experimental techniques for accurate measurement of the state of stress in the structure.

A number of experimental methods have been developed over the years, including both full-field methods such as photoelasticity, moire, holographic interferometry, speckle interferometry, etc., and point-by-point methods such as electrical resistance strain gages and speckle photography methods. Some of these methods are briefly explained below.

\section{Photoelasticity Method}

Photoelasticity is a method of stress analysis in which a model of the object under design is used. The model is made of special plastic material which possesses desirable strain-optical properties [1]. Photoelasticity depends upon the relationship between the state of stress in a transparent material (the model) and the way in which that material transmits polarized light. The experimentally determined quantity is an observed optical pattern; the observed optical pattern is related to the state of stress (Figure 1a) by using both the optical theory and the theory of elasticity; in 


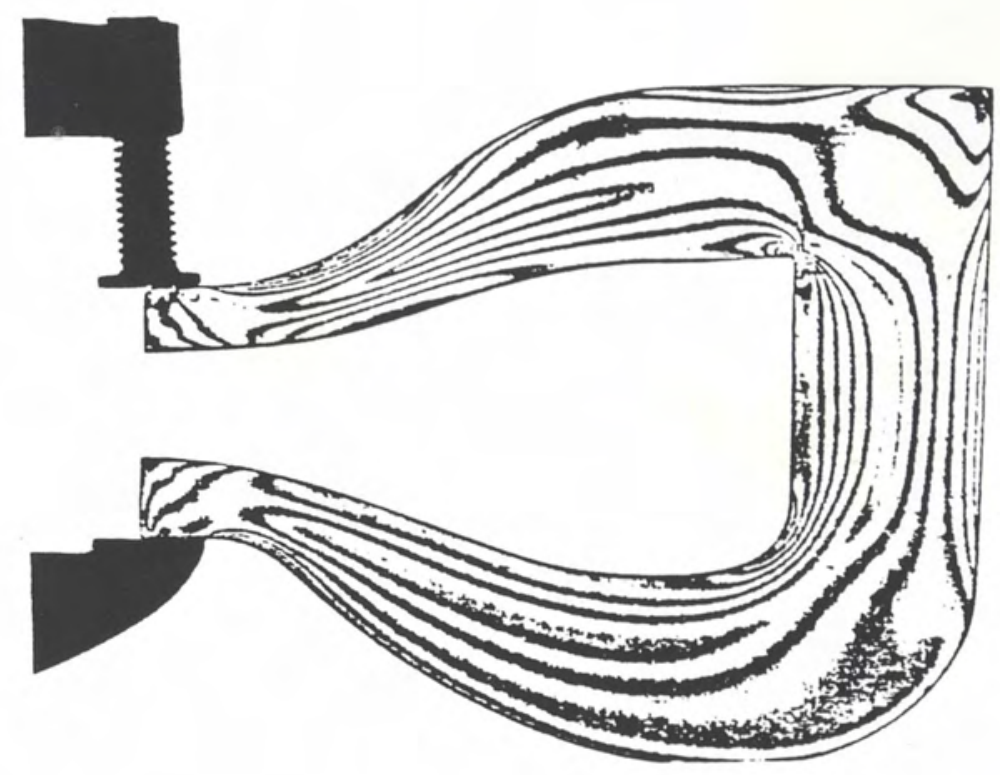

(a)

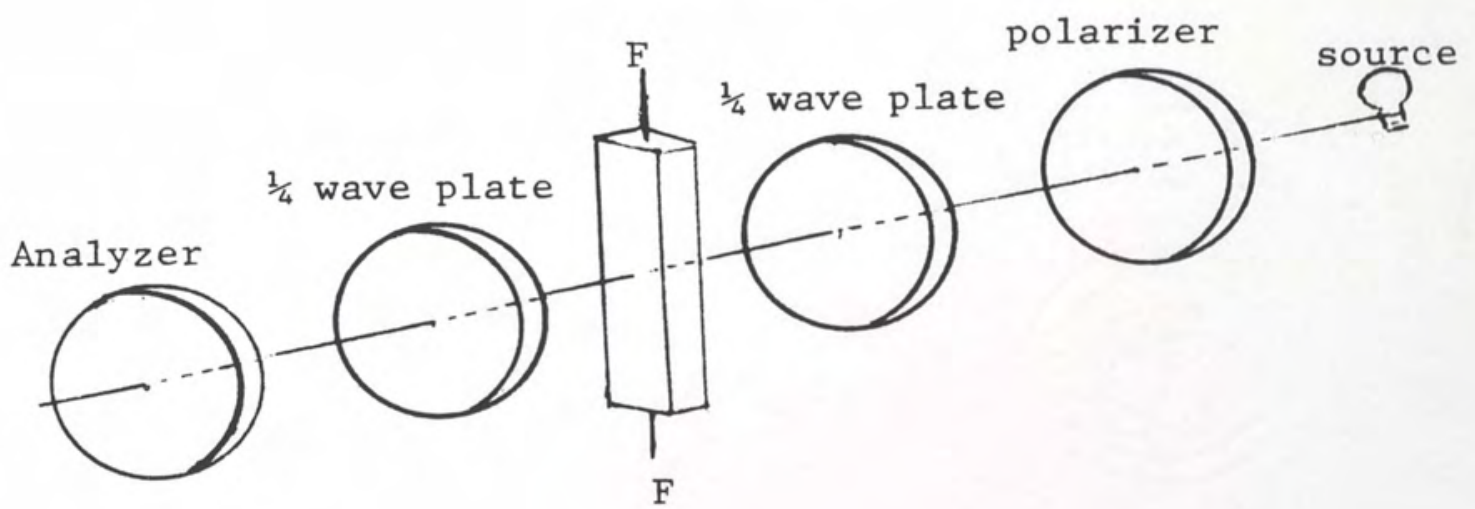

(b)

Figure 1. a) Fringe formation in a model under stress [1]. b) A circular polariscope. 
addition the state of stress in the model must be related to the state of stress in the prototype by using appropriate model theory. Since the model is geometrically similar to the actual structure, the stress distribution in the model indicates the effects of geometry on the stress distribution in the actual structure [1]. Figure 1b shows the arrangement of elements in a circular polariscope.

\section{Moire Method}

The word moire is a French name for a fabric known as watered silk, which exhibits patterns of light and dark bands. This moire effect occurs whenever two similar but not quite identical arrays of equidistant lines or dots are arranged so that one array can be viewed through the other.

In stress analysis work, arrays consisting of straight parallel lines (ideally, opaque bars with transparent interspaces of equal width) are most commonly used. Such arrays are frequently referred to as grids or gratings [2]. Such grating must be applied to the area of interest on the object (photographic printing is most often used); another set of grid applied to a transparent sheet will be laid beside the first set. After applying stress to the object, the relative displacement can be measured using a micrometer or a microscope [3].

The moire fringe phenomenon can be explained in terms of the array of lines shown in Figure 2. The linear sets shown in Figure $2 \mathrm{a}$ are assumed to be identical; one set $\mathrm{S}$ is on the surface to be 


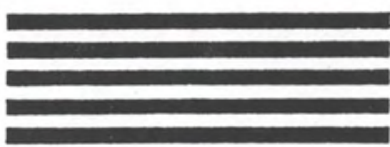

(S)

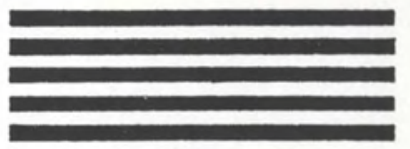

$(\mathrm{M})$

(a)

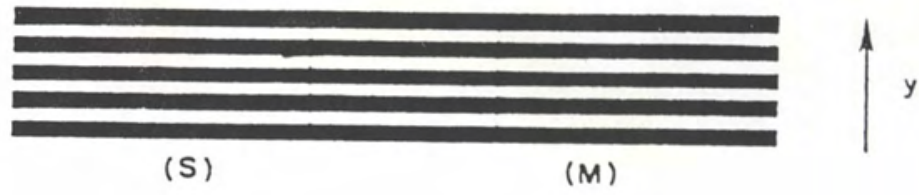

(b)

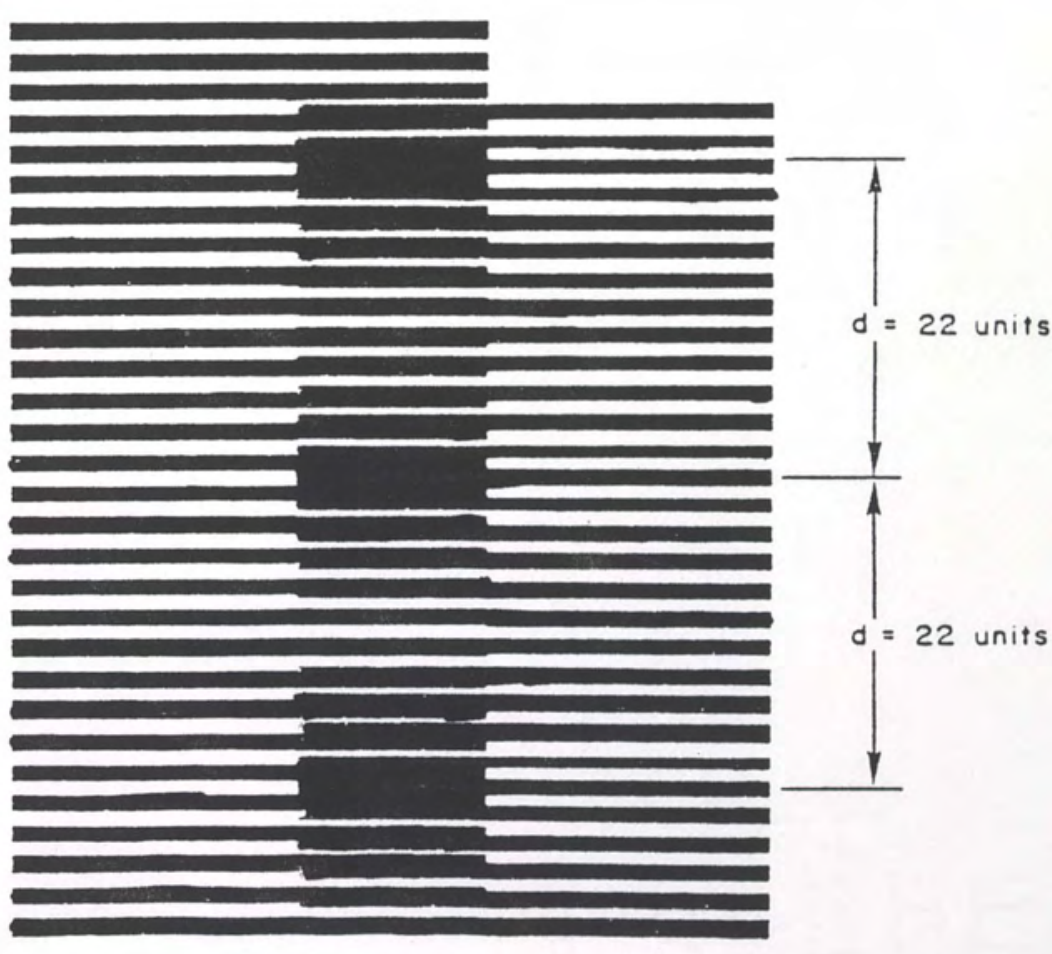

(c)

Figure 2. a) $M$ and $S$ arrays. b) $M$ and $S$ arrays set beside each other c) Relative displacement between $M$ and $S$ arrays [3I. 
studied, the other set $M$, applied to a transparent sheet (the opaque line width and the transparent space width are equal). Now assume that the master grid, $M$, is laid beside the $S$ grid with all lines parallel, as in Figure 2b. After applying uniform strain to the surface, S, the two grids will move relative to each other (Figure $2 \mathrm{c}$ ). In general, the distance, $d$, between the centers of adjacent lines of maximum density will be given by [4]:

$$
\mathrm{d}=\frac{\mathrm{P}}{\varepsilon}(1+\varepsilon)
$$

where:

$$
\begin{aligned}
& \mathrm{d}=\text { the fringe spacing } \\
& \mathrm{P}=\text { the unstrain grid pitch } \\
& \varepsilon=\text { the strain perpendicular to the grid lines }
\end{aligned}
$$

Or,

$$
\varepsilon=\frac{P}{d-P}
$$

The moire fringe method is a "whole field" method, which means that the strain field can be measured completely [2].

\section{Holographic Interferometry Method}

Invention of the laser in 1960 gave a new dimension to the science of optics, and injected practical significance into the study of coherent light [5]. The most important characteristic of laser light, which is used in holography, is its high coherence [6]. 
Holography is an interference method of recording the light waves diffracted by a subject illuminated with coherent light. The diffracted waves are made to interfere with a phase-related reference wave. If the waves are highly coherent, the relative phase between subject and reference wave remains constant in time producing an observable effect on the intensity distribution of the resulting pattern [5]. Figure $3 a$ shows the recording set-up of an off-axis hologram and figures $3 b$ and $3 c$ show its reconstruction set-up [6].

Through the use of off-axis holography, one can produce three-dimensional images of diffusely reflecting objects which appear to be overlaid by interference fringes that are indicative of deformation, displacement or rotation of the object.

For the purpose of strain measurement, the light scattered by the object is recorded during an initial exposure of an off-axis hologram; after deformation, a second hologram is recorded on the same film plate. Upon reconstruction, the film plate is illuminated by a reference laser light as shown in figures $3 b$ and $3 c$. Looking through the hologram, a series of dark and bright fringes are observed. Figure $4 \mathrm{a}$ shows the normal deformation of a cantilever beam subjected to end transverse force [6]. Next, by assigning fringe order numbers to the bright fringes (Figure 4b), and utilizing the following equation [6]:

$$
w(x)=\frac{N \lambda}{2}
$$




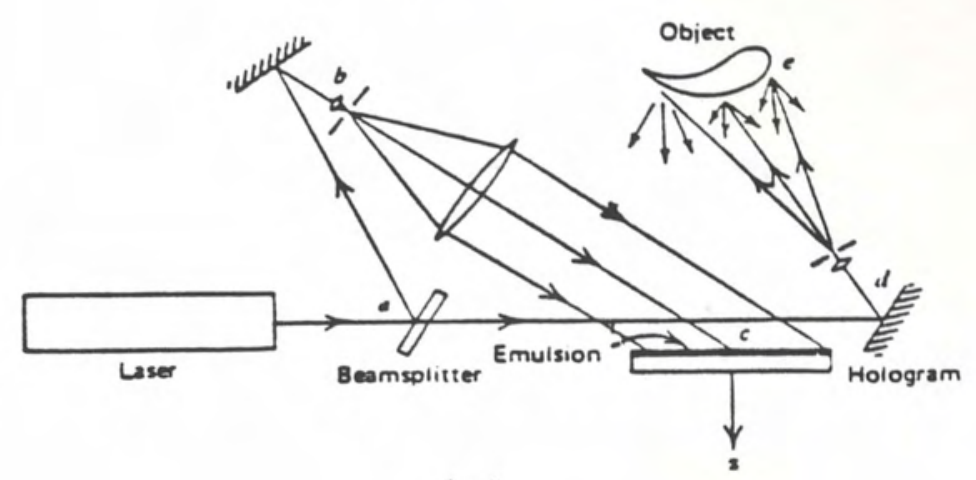

(a)

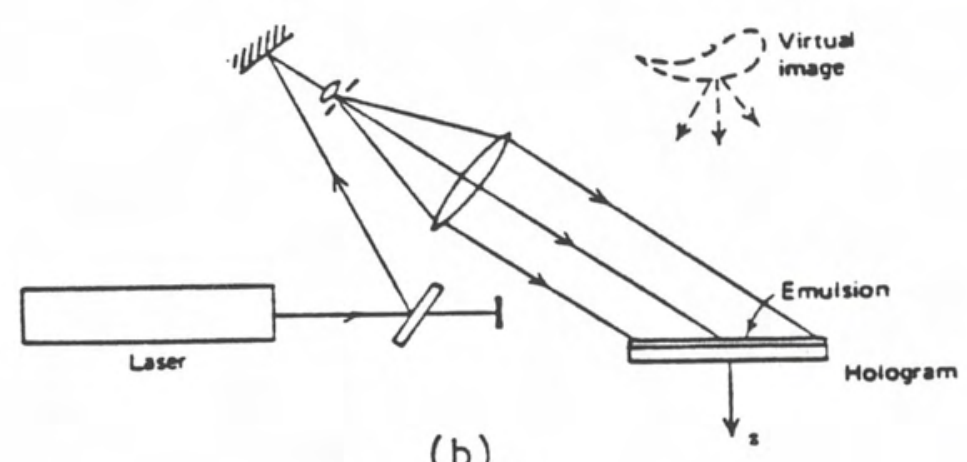

(b)
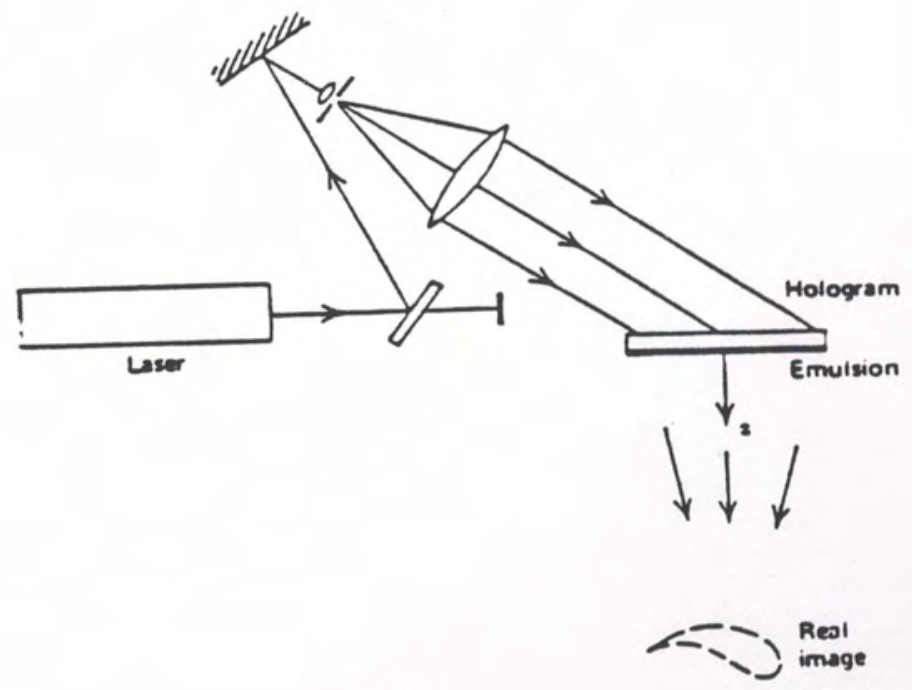

(c)

Figure 3. Off-axis holography. a) System for recording the hologram. b) Reconstruction of the true, virtual image.

c) Reconstruction of conjugate, real image [6]. 


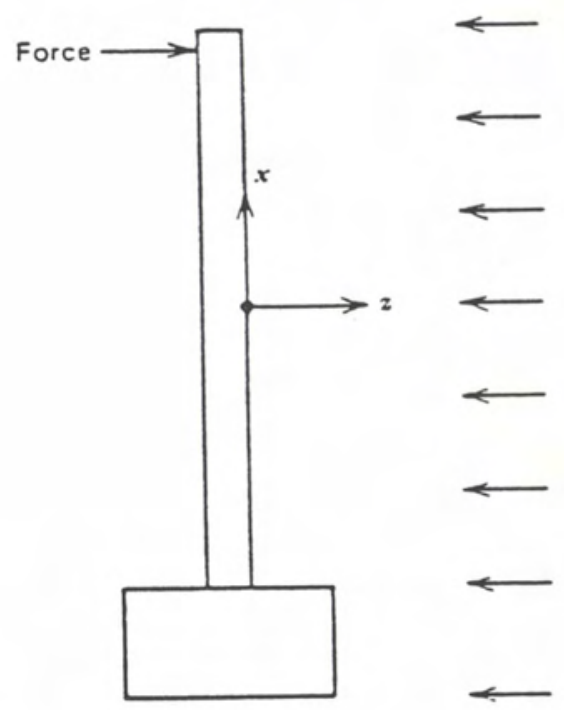

(a)

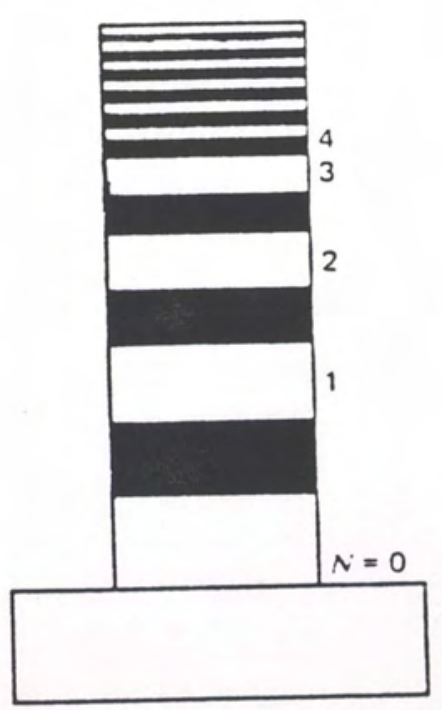

(b)

Figure 4. Normal deformation of a cantilever beam: a) Side view of the beam. Displacement is in the $+z$ direction, and illumination in the $-z$ direction. b) Fringe pattern observed on the front of the beam [6]. 
where:

$$
\begin{aligned}
& \mathrm{w}(\mathrm{x})=\text { deformation of the } \mathrm{x} \text { location } \\
& \mathrm{N} \quad=\text { fringe order } \\
& \lambda \quad=\text { wavelength of laser light }
\end{aligned}
$$

Using equation (3), the deformation or displacement at any location of the object can be calculated.

Holographic interferometry is only sensitive to out-of-plane deformation. For in-plane deformation, speckle photography is more sensitive.

\section{Electrical-Resistance Strain Gage Method}

Theoretically, an electrical-resistance strain gage operation is based on the principle that the electrical resistance of a conductor changes when it is subjected to mechanical deformation, therefore generating an electric signal. Magnification of this signal is then accomplished by electric means, such as an amplifier. Typically, an electric conductor is bonded to the specimen with an insulating cement under no-load conditions. A load is then applied, which produces a deformation in both the specimen and the resistance element. This deformation is indicated through the use of a strain gage indicator, or a measurement of the change in resistance of the element and a calculation procedure shown below in equation (4) [7]:

$$
\varepsilon=\frac{1}{F} \frac{\Delta R}{R}
$$


where:

$$
\begin{aligned}
& \varepsilon=\text { local strain } \\
& F=\text { gage factor } \\
& \Delta \mathrm{R}=\text { change in resistance } \\
& \mathrm{R}=\text { original resistance of the gage }
\end{aligned}
$$

The important advantage that the electrical resistance type strain gage has is the relative ease with which the output can be amplified, displayed and recorded [2]. Figure 5 shows some of the electrical resistance strain gages currently in use.

This method is a point-by-point method, which means that the strain field of the object cannot be completely measured using only one gage.

\section{Speckle Photography Method}

Speckle photography is a technique for making moderately-sensitive measurements of in-plane translation, strain, rotation and vibration [6]. Its principle attractions are the simplicity of the optical system and the relative ease of displaying and interpreting the results. As shown in Figure 6a, the object is illuminated by laser light. It is then photographed twice; once before and once after deformation on the same film plate. A narrow beam of laser light is then directed through the processed film plate (Figure 6b), producing fringes in the back focal plane of the lens (Figure 6c). By knowing the distance between successive fringes, the wavelength of the laser light and the distance between the film plate and the viewing plane, 


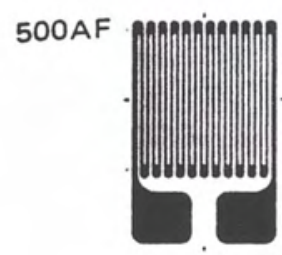

750DT
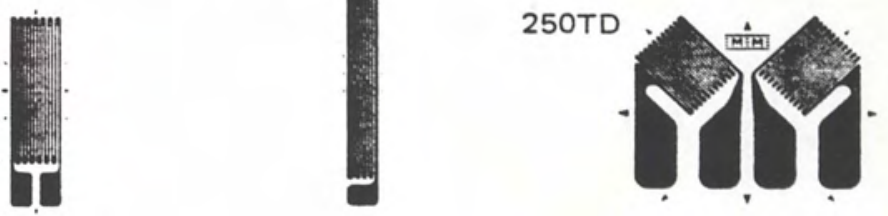

250TA
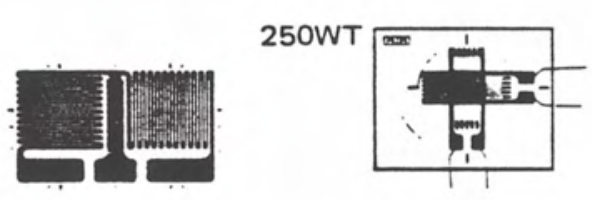

250RA

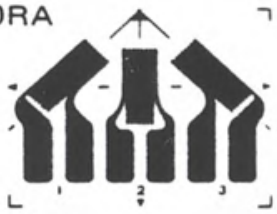

250YA

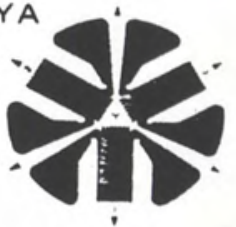

250WY

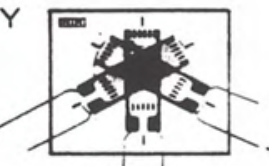

250TK

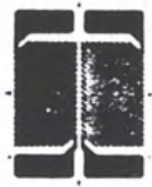

455JB

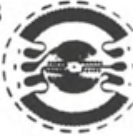

250NA

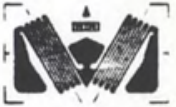

Figure 5. Configuration of metal-foil strain gages [7]. 


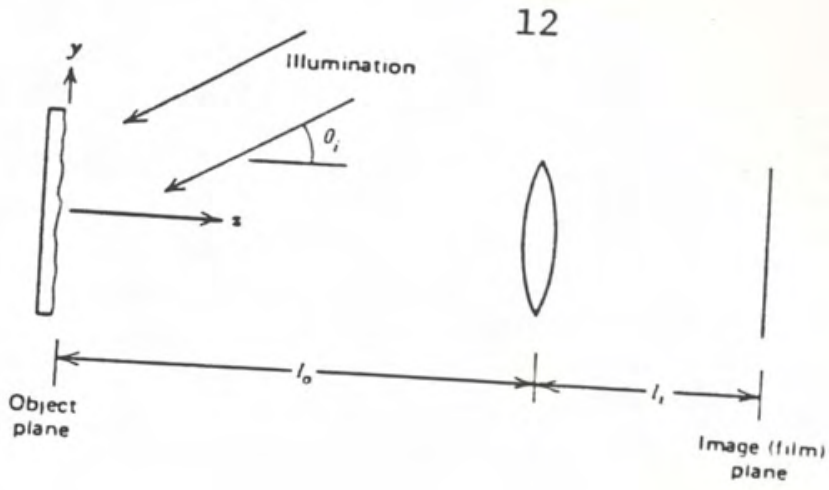

(a)

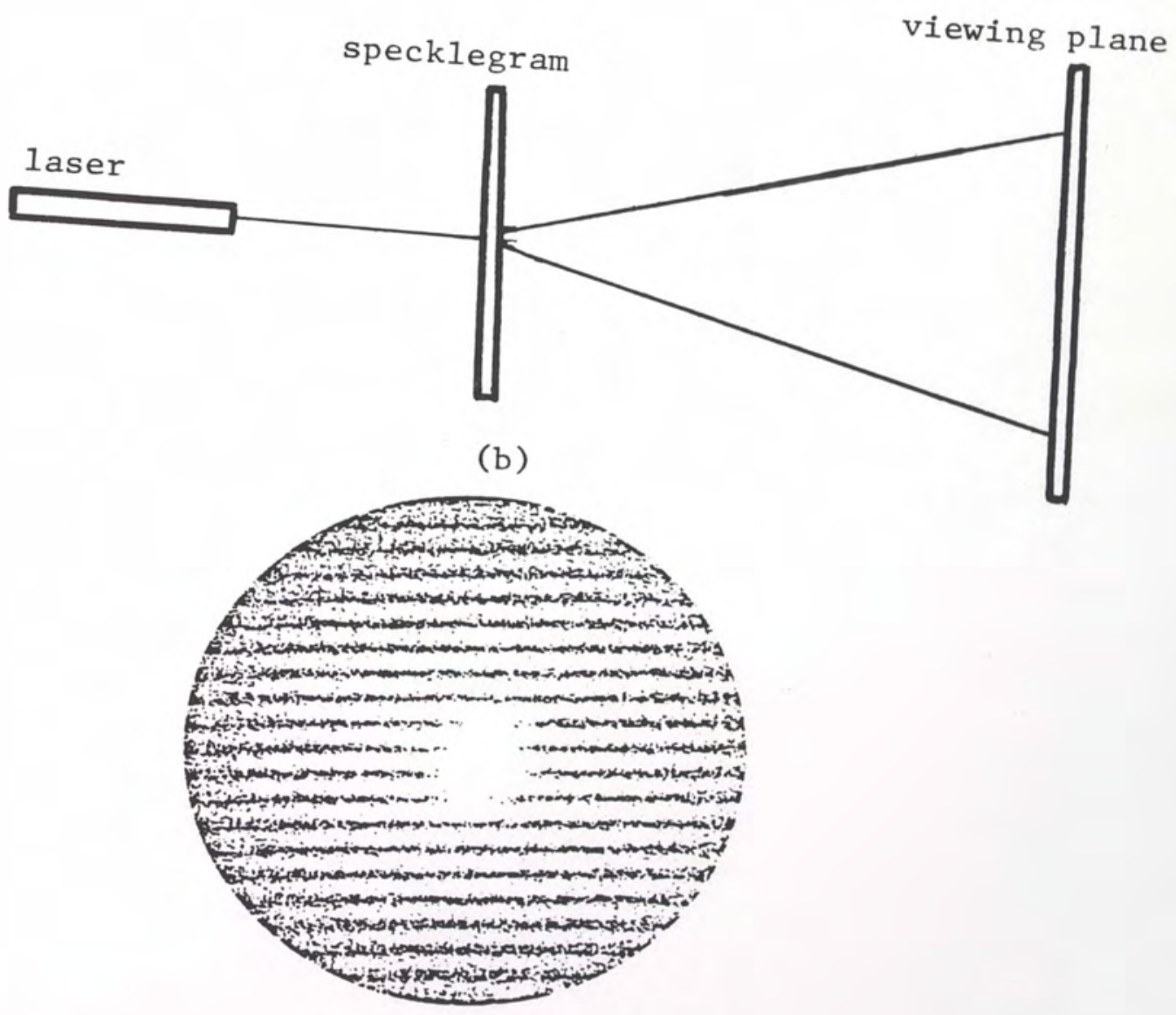

(c)

Figure 6. Two-exposure speckle photography for measurement of in-plane formation.

c) Formation of fringe b) Set-up for fringe 
the deformation of the object can be calculated using the following equation [6]:

$$
\mathrm{U}=\frac{\lambda z}{\mathrm{Md}}
$$

where:

$$
\begin{aligned}
& \mathrm{U}=\text { in-plane object displacement } \\
& \lambda=\text { wavelength of light used in producing the fringes } \\
& \mathrm{z}=\text { distance between the specklegram and the viewing plane } \\
& \mathrm{d}=\text { fringe spacing } \\
& \mathrm{M}=\text { magnification factor of the recording camera }
\end{aligned}
$$

Also, by knowing the fringe orientation angle, $\theta$ (Figure 7), displacement components, $\mathrm{U}_{\mathrm{x}}$ and $\mathrm{U}_{\mathrm{y}}$, may be calculated as follows:

$$
\begin{aligned}
& \mathrm{U}_{\mathrm{x}}=\mathrm{U} \cdot \sin \theta \\
& \mathrm{U}_{\mathrm{y}}=\mathrm{U} \cdot \cos \theta
\end{aligned}
$$

The scope of this research report is to develop an automated method for direct measurement of in-plane displacements of structures subjected to different loading conditions. The speckle photography technique is used. The obtained fringes from the optical Fourier filtering of the processed film plate at a point through the use of a thin laser beam was then digitized using a CCD 133 charge linear diode array. The digitized data is Fourier transformed using a spectrum analyzer and then analyzed by a computer to obtain the in-plane displacement at a point on the surface of the structure. 


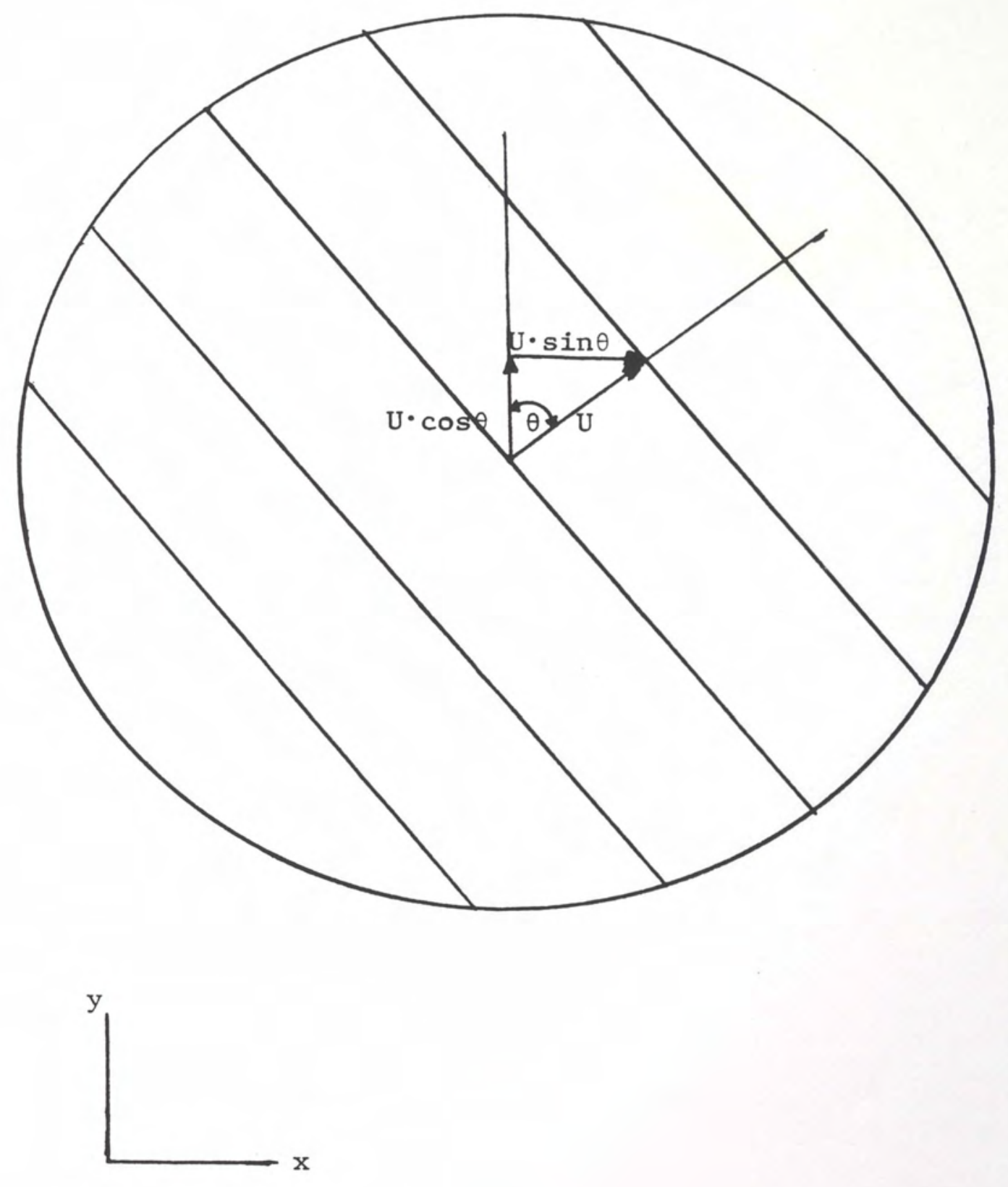

Figure 7. Diagram of fringe orientation and the respective $x$ and $y$ components of fringe spacing. 
Measurement of deformations at different points of the structure is possible using a computer-controlled translation stage. 
CHAPTER II

LITERATURE REVIEW

The method of wavefront reconstruction (holography) was first invented by Dennis Gabor in 1948 [8]. Gabor invented this method to improve the resolution in images obtained with an electron microscope. Although unable to demonstrate the validity of his principle with electron waves, he was able to do so with visible light.

Holography, after a decade, seemed destined for obscurity. This brightened in the early 1960s with work carried out at the University of Michigan Institute of Technology. Leith and Upatnieks [9] began publication of a series of reports demonstrating a new approach to optical holography based on communication theory techniques. By 1964, they had convincing proof that holography was practical and that the use of laser light was an important factor [10].

The application of holography to interferometry was first suggested by Horman [11], who described the inclusion of a hologram. Holographic interferometry of diffusely scattering objects was introduced in the context of vibration analysis by Powell and Stetson in March 1965 [12]. The development of two-exposure and real-time holographic interferometry occurred independently in several laboratories, and was reported in papers submitted for publication during the summer of 1965. This work includes that of Burch [13], 
Collier, Doherty and Pennington [14]; Stetson and Powel1 [15]; and Haines and Hildebrand [16] who studied the deformation and displacement of diffusely reflecting objects; and that of Heflinger, Wueker and Brooks $[17,18]$, who used the method for aerodynamic me asurements.

The technique of holographic addition and subtraction was also introduced in 1965 by Gabor et al. [19], and subsequently was noted to be equivalent to holographic interferometry.

In-plane strain measurements were considered by Aleksandrov and Bonch-Brevich [20] and Ennos [21] in 1966 and was used by Wilson [22] in 1967 to measure displacement in a rectangular membrane with a hole in the center subjected to a uniform tensile stress in the longitudinal direction.

In 1967, Barnett [23] presented a sequence of time-average interferograms of vibration of a circular plate with clamped edge. He noted that some of these interferograms appeared to display nodal patterns that were not in agreement with the classical theory of vibrating plates. Molin and Stetson [24] demonstrated that such patterns actually represented combinations of classical modes.

Subsequent analysis and experimentation by Stetson [25], Wilson [26] and their co-workers have produced a detailed description of the formation of fringes in time-average holographic interferometry of objects executing complex motion, particularly multiple vibrational modes. 
Introduction of speckle interferometry in 1969 opened new research areas, so that there now exists quite a collection of techniques that are applicable to a wide variety of problems. Any motion on the object surface brings about the motion of speckle pattern, as was explained by Tiziani [27] and Archbold and Ennos [28] in 1970. They concluded that speckles in the image of an object will either shift their locations as the object moves, or they will change their pattern, or both. This conclusion was then carried further, leading to the concept of double-exposure speckle photography. This is the simplest technique in speckle photography and was discussed by Archbold [29], Tiziani [30] and Kopf [31] in 1975.

The double-exposure speckle photography provides information related to the state of strain of the object under consideration. Analysis of such a double-exposure photograph is carried out by directing a narrow laser beam through the photographic film plate at normal incidence to generate in the far field its Fourier transform. This analysis was conducted by Tiziani [30] in 1972. This takes the form of a diffraction halo modulated by a pattern of parallel equispaced fringes, similar to Young's fringes. Fringes are perpendicular to the direction of the displacement and they have spacing inversely proportional to the object displacement.

Stetson [32] has analyzed the apparent motion of speckles due to arbitrary object displacement and deformation, and has concluded that by recording two double-exposed specklegrams from the same observation direction, but with the camera focused on different 
planes, the object translation normal to that direction can be extracted.

Adams and Maddux [33] and Boone [34] have discussed the possibility of combining holographic interferometry and speckle photography techniques for use in stress analysis, since the former technique is most sensitive to deformations normal to the object surface and the latter is most sensitive to deformation in the plane of the object surface.

Hudson and Setopoulous [35], in 1974, demonstrated a system for recording sequential pairs of speckle photography in order to measure time-dependent deformations, such as creep.

In 1978, Maddux [36] utilized mechanical scanning of the fringe by a large photodetector. Ineichen [37], in 1979, used a television pick-up system for data acquisition, and also Kaufmann, Ennos, Gale and Pugh [38] used a compact optical system that projected the 1-D Fourier transform of a small area of the specklegram, without distortion, onto a self-scanning linear photodiode array.

Kaufmann [39] used an automated read-out system to directly process the digitized fringe pattern.

In 1981, Ichirou Yamaguchi [40] presented a method based on theoretical investigations of speckle displacement induced by small object deformation. He also used an electronic speckle correlation with a photodiode array to measure speckle displacement. By this method, the strain measurement at a small illuminated area was possible in real-time. In 1982, Yamaguchi [40] used a double-beam arrangement 
with a linear sensor of high resolution (square pixels of a size of 15 microns on a side) to convert the intensity data of speckles to an analog-ensembled signal. A data ensemble was then digitized and stored while the object was strained. Then, the image of strained object was digitized and stored. He then cross-correlated the two signals to yield a point of high correlation.

In 1983, Yamaguchi [41] discussed the formation of Young's fringes that are observed in speckle photography. He derived both the shape and the contrast of the fringes by starting from the crosscorrelation function of the light intensities to be recorded in a double-exposure specklegram. He also discussed the effect of focusing and defocusing of the specklegram recording which affected the speckle shape, as well as the origin of speckle decorrelation affecting the contrast.

In 1984, A. Muramatsu and M. Lunazzi [42] used correlation techniques between a speckle pattern and its photographic negative to measure lateral displacements and vibrations of rigid objects. 


\section{CHAPTER III}

\section{THEORY OF FRINGE FORMATION}

Fringe formation on the back focal plane of a lens in speckle photography can be explained by Thomas Young's two-slit experiment, as shown in Figure 8. Light waves from slit A reach slit S1 and S2 at the same time and these originate from a single source. Therefore, the secondary wavelengths leaving S1 and S2 are in phase or coherent. Figure 9 shows how the light and dark bands are produced. The bright bands occur whenever the waves arriving from the two slits interfere constructively (the amplitude of resultant wave is greater than the amplitude of either component wave). Dark bands occur when destructive interference (the resultant amplitude is smaller than either wave) is experienced. At point B in the center of the screen, light travels the same distance from each slit. The difference in path length is zero and constructive interference results in a bright central band. At point $C$, the difference in path length causes the waves to interfere destructively. Another bright band occurs at point D when the waves from each slit again reinforce each other [43]. Theoretical conditions necessary for the production of bright and dark bands are as follows. Consider light reaching point $D$ at a distance $y$ from the central axis $A B$, as shown in Figure 10. The separation of the two slits is represented by $d$, and the screen is located at a distance $x$ from the slits. Point $D$ on the screen makes 


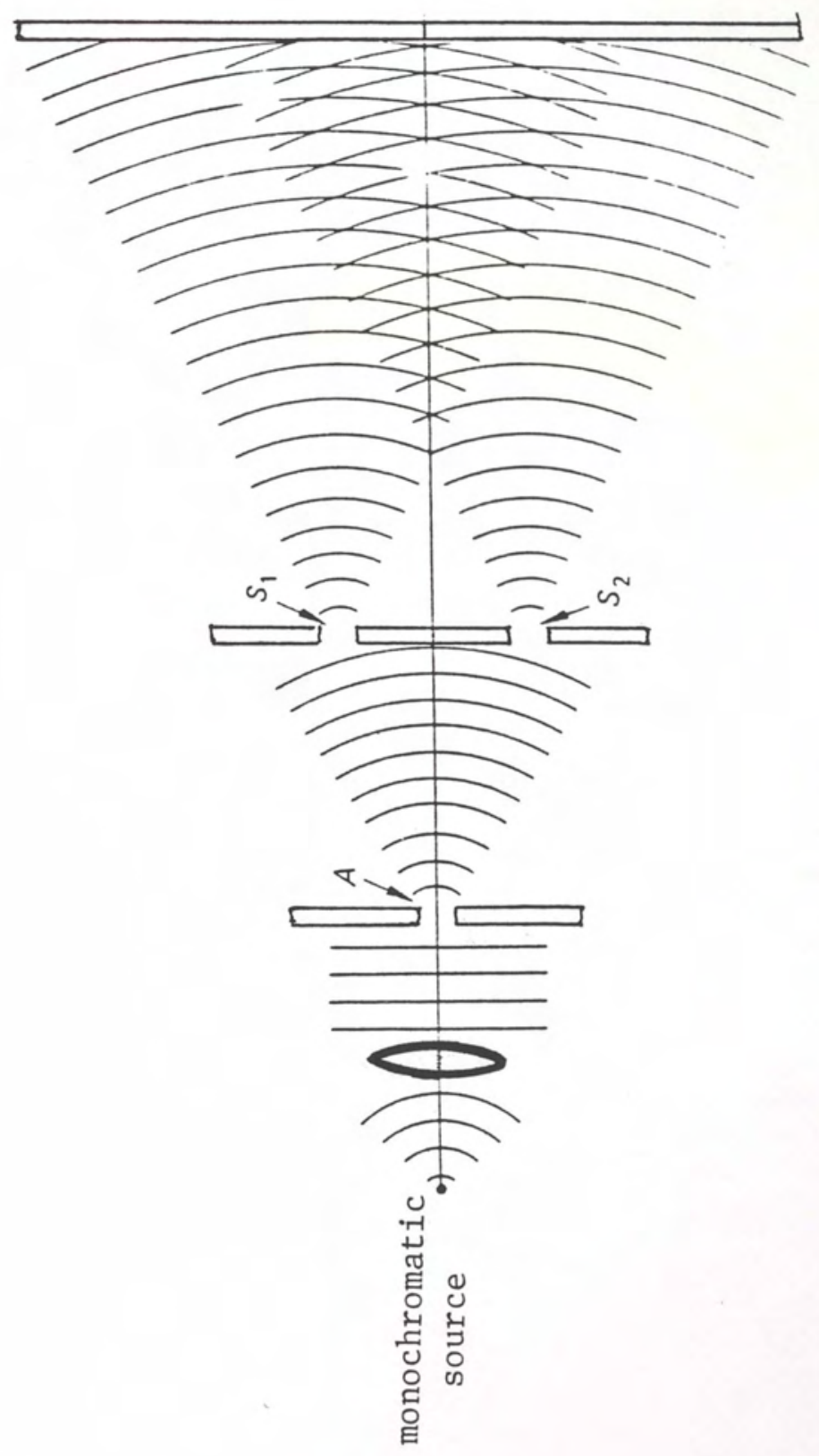

Figure 8. Illustration of Young's experiment [43]. 


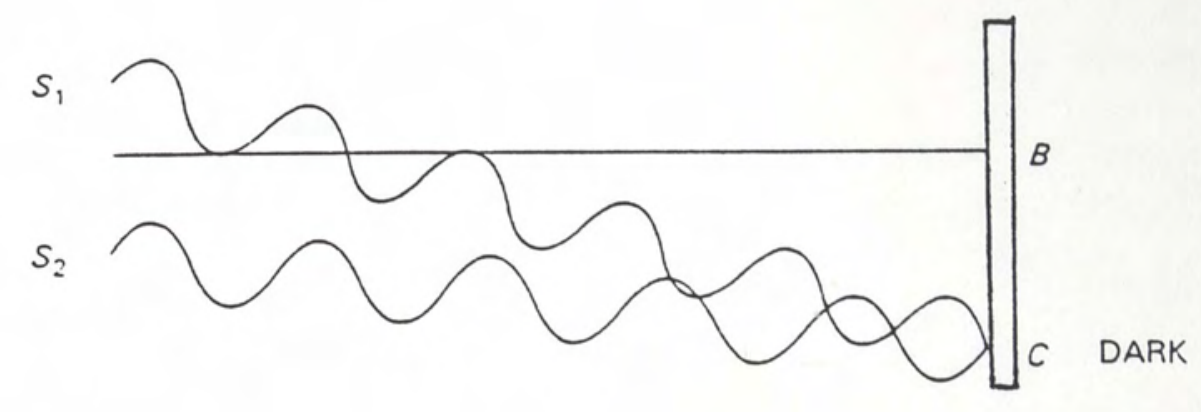

(a)
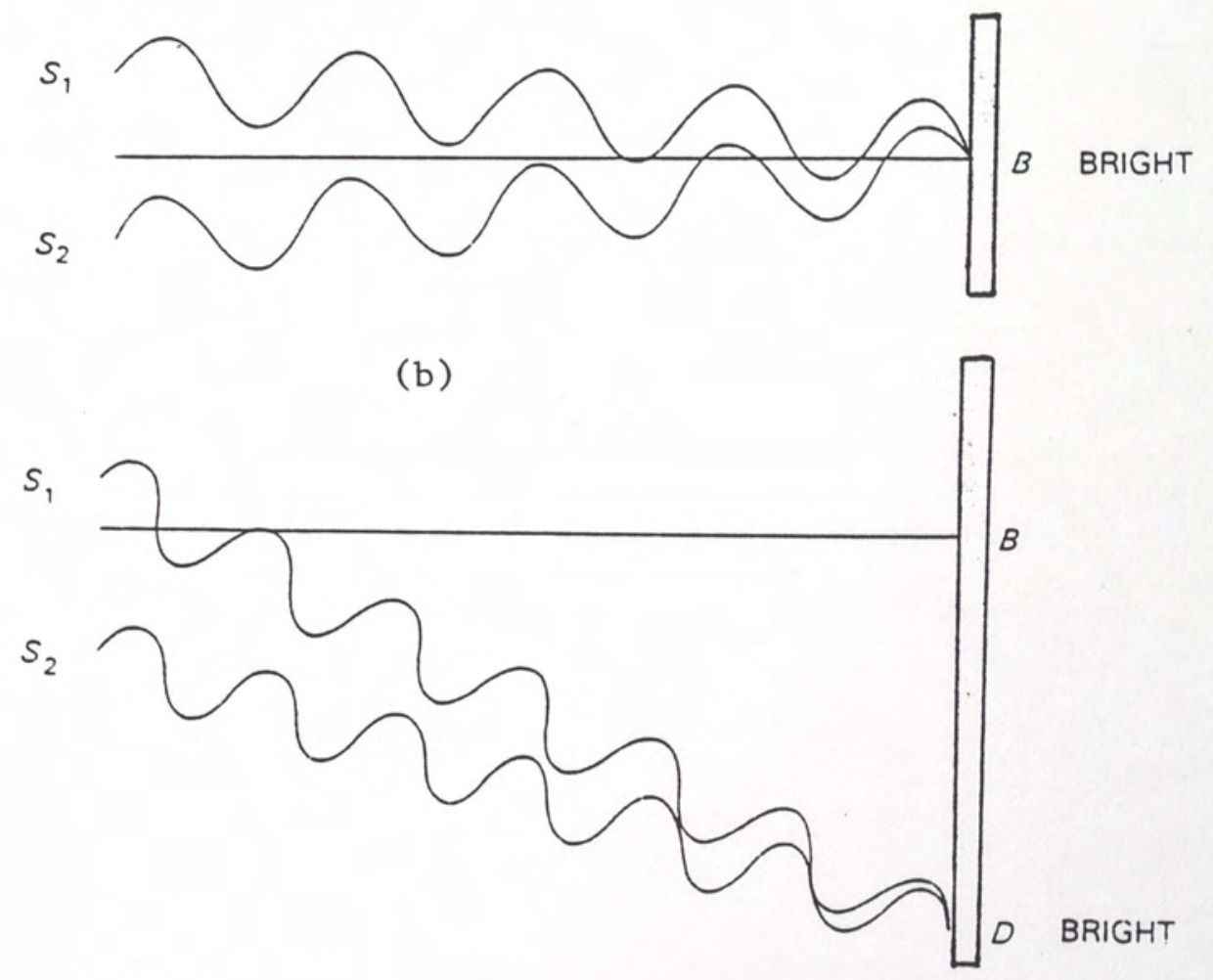

(c)

Figure 9. Origin of light and dark bands in an interference pattern [43]. 


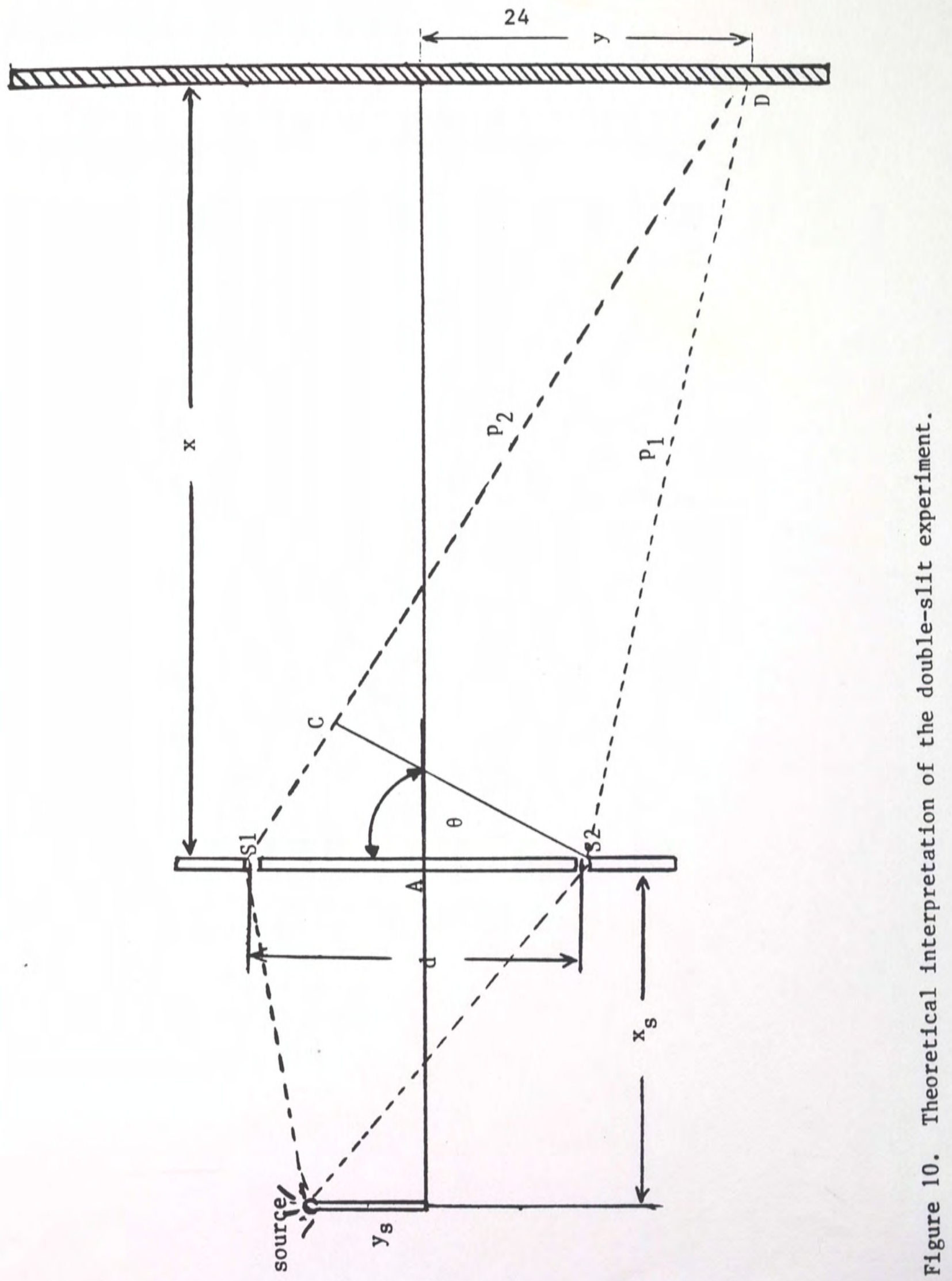


an angle $\theta$ with the axes of the system. The line S2C is drawn so that the distances $C D$ and $P 2$ are equal. As long as the distance $x$ to the screen is much greater than the slit separation d, P1, P2 and DA may be considered to be perpendicular to the line $\mathrm{S}_{2} \mathrm{C}$. Therefore, the angle ${ }_{L} \mathrm{~S}_{1} \mathrm{~S}_{2} \mathrm{C}$ is equal to the angle $\theta$, and the difference $\Delta \mathrm{P}$ in path length of light coming from $\mathrm{S} 1$ and $\mathrm{S} 2$ is given by:

$$
\Delta \mathrm{P}=\mathrm{P} 1-\mathrm{P} 2=\mathrm{d} \sin \theta
$$

Constructive interference will occur at D when this difference in path length is equal to:

$$
0, \lambda, 2 \lambda, 3 \lambda, 4 \lambda, \ldots, \mathrm{n} \lambda
$$

where $\lambda$ is the wave length of light. Therefore, the conditions for bright fringes are given by:

$$
\mathrm{d} \cdot \sin \theta=\mathrm{n} \lambda \quad \mathrm{n}=0,1,2,3, \cdots
$$

where $d$ is the slit separation and $\theta$ is the angle that the fringe makes with the axis.

The condition required for the formation of dark fringes at D will be satisfied when the path difference is:

$$
\frac{\lambda}{2}, \frac{3 \lambda}{2}, \frac{5 \lambda}{2}, \frac{7 \lambda}{2}, \cdots
$$


Under these conditions, destructive interference will cancel the waves. Thus, dark fringes will occur when:

$$
\mathrm{d} \cdot \sin \theta=\mathrm{n} \cdot \lambda / 2 \quad \mathrm{n}=1,3,5, \ldots
$$

Equations (9) and (10) may be put into a more useful form by expressing them in terms of the measurable distances $x$ and $y$ For small angles:

$$
\sin \theta \simeq \tan \theta=y / x
$$

Substituting equation (11) into equations (9) and (10) yields:

$$
\begin{array}{ll}
\text { Bright fringes: } & \frac{\mathrm{d}(\mathrm{y})}{\mathrm{x}}=\mathrm{n} \lambda \quad \mathrm{n}=0,1,2,3, \ldots \\
\text { Dark fringes: } & \frac{\mathrm{d}(\mathrm{y})}{\mathrm{x}}=\mathrm{n} \frac{\lambda}{2} \quad \mathrm{n}=1,3,5, \ldots
\end{array}
$$

To find the intensity of light at the plane of fringes, consider the same experiment. Using the electromagnetic theory, the irradiance at the plane of fringes becomes [6]:

$$
\begin{aligned}
I & =2 \cdot I_{0}(1+\cos \delta) \\
& =4 \cdot I_{0} \cdot \cos ^{2}(\delta / 2)
\end{aligned}
$$

where:

$$
\begin{aligned}
I= & \text { irradiance at the fringe plane } \\
I_{0}= & \text { combined irradiance from both slits } \\
\delta= & \text { phase difference }=(2 \pi / \lambda) \Delta 1 ; \text { where } \Delta 1 \text { is the difference in } \\
& \text { distance the } 1 \text { ight travels from the source } \mathrm{S} \text { the } \\
& \text { observation point at } \mathrm{y}, \text { and } \lambda \text { is the wavelength of light }
\end{aligned}
$$


From geometry, $\Delta 1$ can be presented as:

$$
\begin{aligned}
\Delta 1 & =\left\{\left[x_{s}^{2}+\left(\frac{d}{2}-y_{s}\right)^{2}\right]^{\frac{1}{2}}+\left[x_{s}^{2}+\left(\frac{d}{2}-y\right)^{2}\right]^{\frac{1}{2}}\right\} \\
& -\left\{\left[x_{s}^{2}+\left(\frac{d}{2}+y_{s}\right)^{2}\right]^{\frac{1}{2}}+\left[x^{2}+\left(\frac{d}{2}+y\right)^{2}\right]^{\frac{1}{2}}\right\}
\end{aligned}
$$

Considering that $y_{S}, y$ and $d$ are much smaller than $x$ and $x_{S}$, equation (15) can be approximated as:

$$
\Delta 1=-\left(\frac{d y}{x_{s}}+\frac{d y}{x}\right)
$$

The irradiance is then:

$$
I=4 I_{0} \cos ^{2}\left[\frac{\pi d}{\lambda}\left(\frac{y}{x}+\frac{y_{s}}{x_{s}}\right)\right]
$$

This equation represents parallel fringes (Figure 11) [6].

Local in-plane displacements in the recorded specklegram can be determined by illuminating it with a small laser beam as shown in Figure 12. For the small region of illumination of the specklegram, the displacement is assumed uniform and the region contains pairs of identical speckles displaced by a distance L, the light in the resulting pairs of diffraction cones will interfere to form a diffraction pattern modulated by Young's fringes.

Fringes are perpendicular to the direction of the object displacement $\mathrm{U}(\mathrm{x}, \mathrm{y})$, and they have a spacing, $\mathrm{d}$, inversely proportional 


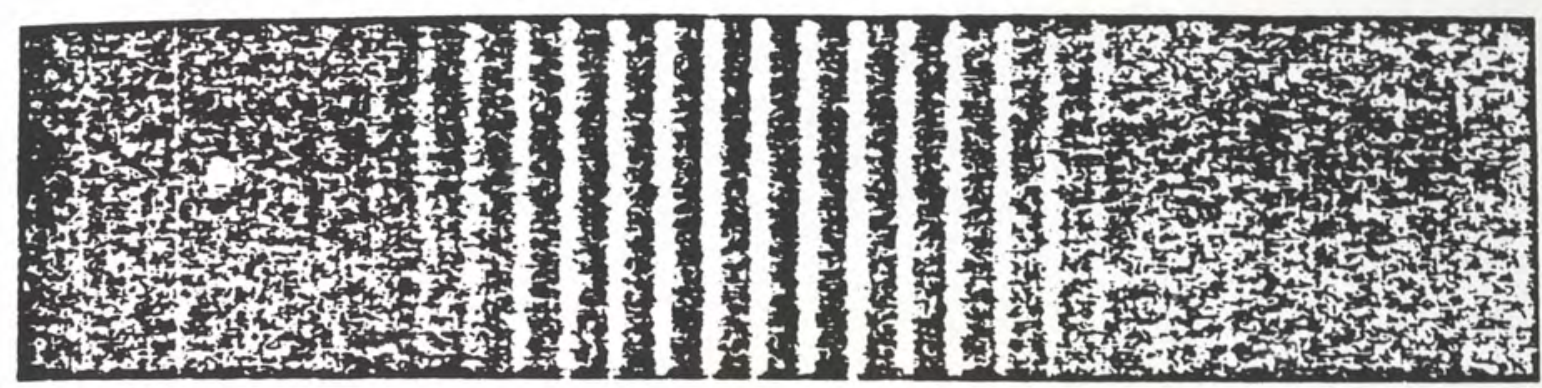

Figure 11. Photograph of an interference pattern in Young's experiment [43].

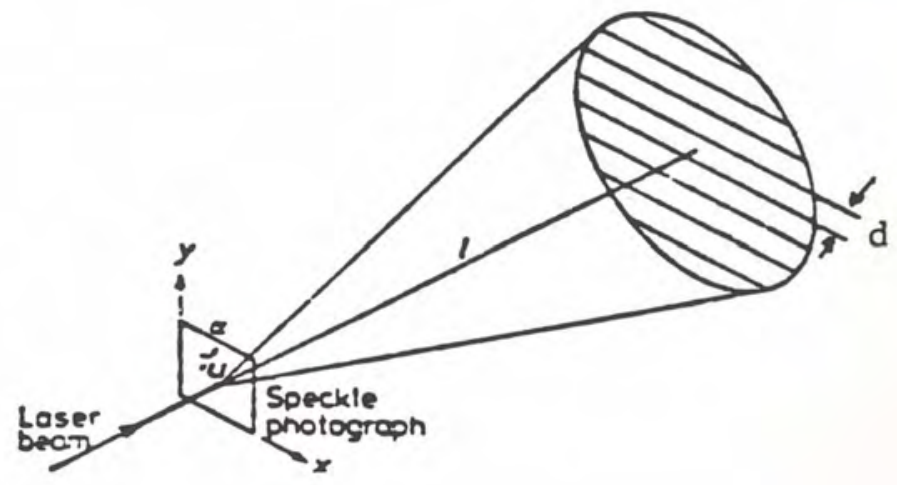

Figure 12. Fringe pattern formation by diffraction at a double exposure speckle photograph [38]. 
to the object displacement, which is given by [6]:

$$
\mathrm{U}(\mathrm{x}, \mathrm{y})=\frac{\lambda \mathrm{z}}{\mathrm{Md}}
$$

where:

$$
\begin{aligned}
& \begin{array}{l}
\lambda= \\
z= \\
\quad \text { effectivength of light source } \\
\text { plane }
\end{array} \\
& M=\text { magnification of imaging system } \\
& d=\text { fringe separation }
\end{aligned}
$$

A Young's fringe pattern is the predominant mode of retrieving the displacement information carried by double-exposure specklegram. The useful parameters are the fringe spacing and the fringe orientation. Since it is a pointwise approach, a great deal of data analysis is involved if one has to analyze a number of specklegrams.

The fringe spacing can be deduced by Fourier transforming the signal obtained from scanning across Young's fringes by a linear photodiode array. The first-order peak (fundamental frequency of the signal) is a representative of the fringe spacing.

The Fourier transform of a function, $g(t)$, is defined by:

$$
X(f)=\int_{-\infty}^{+\infty} g(t) e^{-i 2 \pi f t} d t
$$

To demonstrate the above, two examples are discussed. In the first example, a sinewave of $400 \mathrm{~Hz}$ frequency was generated by a function generator. This signal was then Fourier transformed using the 
spectrum analyzer. Figure 13 shows the time record and the FFT plots of the sinewave, respectively. As shown in Figure 13, the FFT plot, the fundamental frequency is $400 \mathrm{~Hz}$, and it occurs at $1 /$ period of the sinewave. This result was checked by examining another sinewave of $800 \mathrm{~Hz}$ frequency. Figure 14 represents the time record and the FFT plots, respectively, for the second example which justifies the result. 

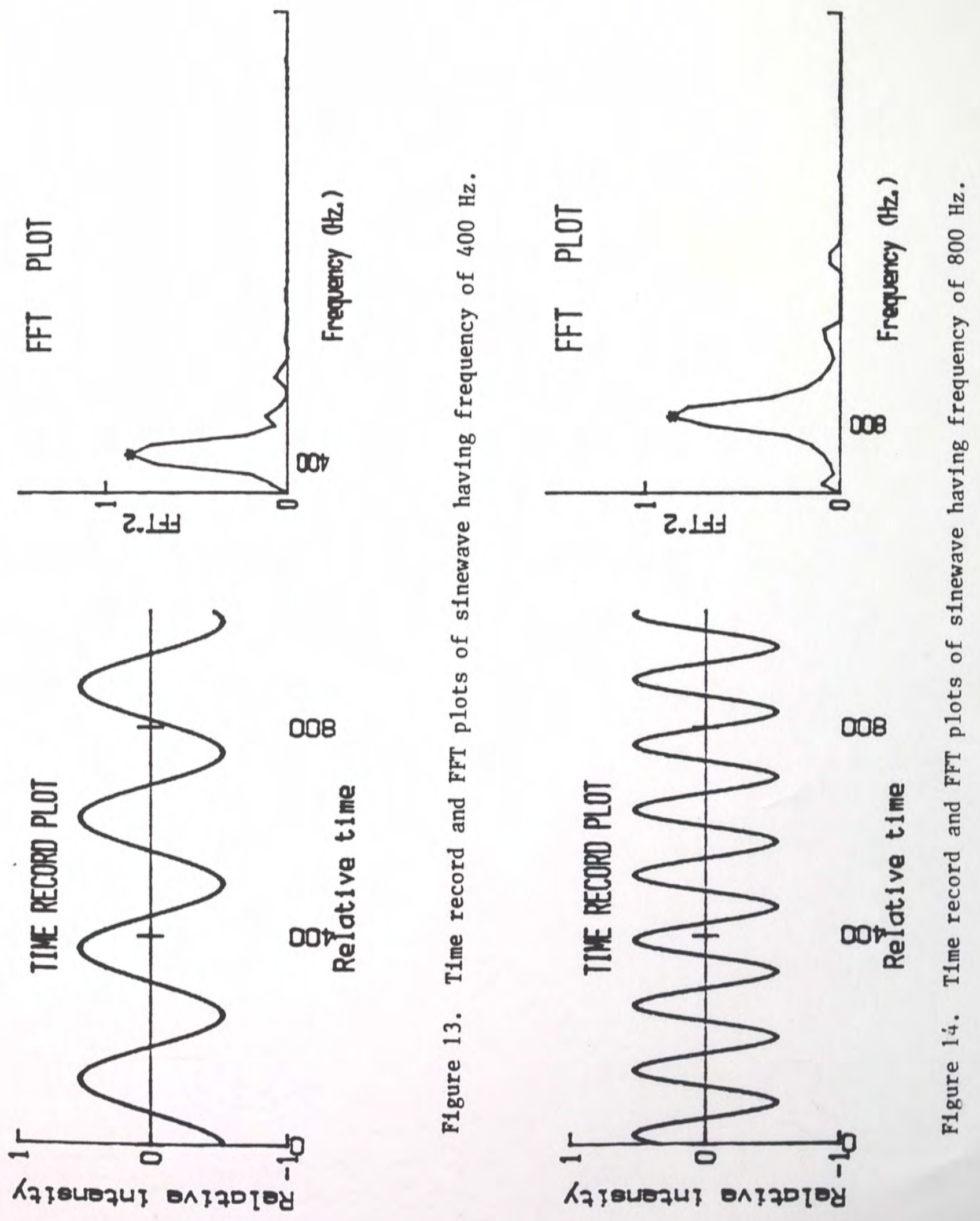


\section{CHAPTER IV}

\section{COMPUTER-AIDED DATA ACQUISITION SYSTEM HARDWARE AND EXPERIMENTAL SET-UP}

In general, automatic processing of Young's fringes consists of three parts: an optical Fourier processor, a fringe pattern digitizer and a fringe pattern analyzer. The first is composed of a low-power laser, a stage to hold the specklegram and a stepping motor to shift the specklegram so that it can be scanned. The second part may be a digitizing TV camera or linear diode sensor. The 1 ast part is a computer with appropriate software to do the computational and the cont rolling tasks.

This chapter presents the hardware used to compute in-plane displacements of objects from double-exposure specklegrams. Figures 15 and 16 illustrate the data acquisition system used in the experimentation. The basic components and their functions are as follows.

\section{$\underline{\text { Laser }}$}

The source of coherent light was a Newport Corporation's He-Ne laser (5 milliwatt output power), with 1.3 millimeter beam diameter and 0.632 micrometer wavelength. Light intensity was controlled by a variable beam splitter. This instrument was placed right after the laser. 


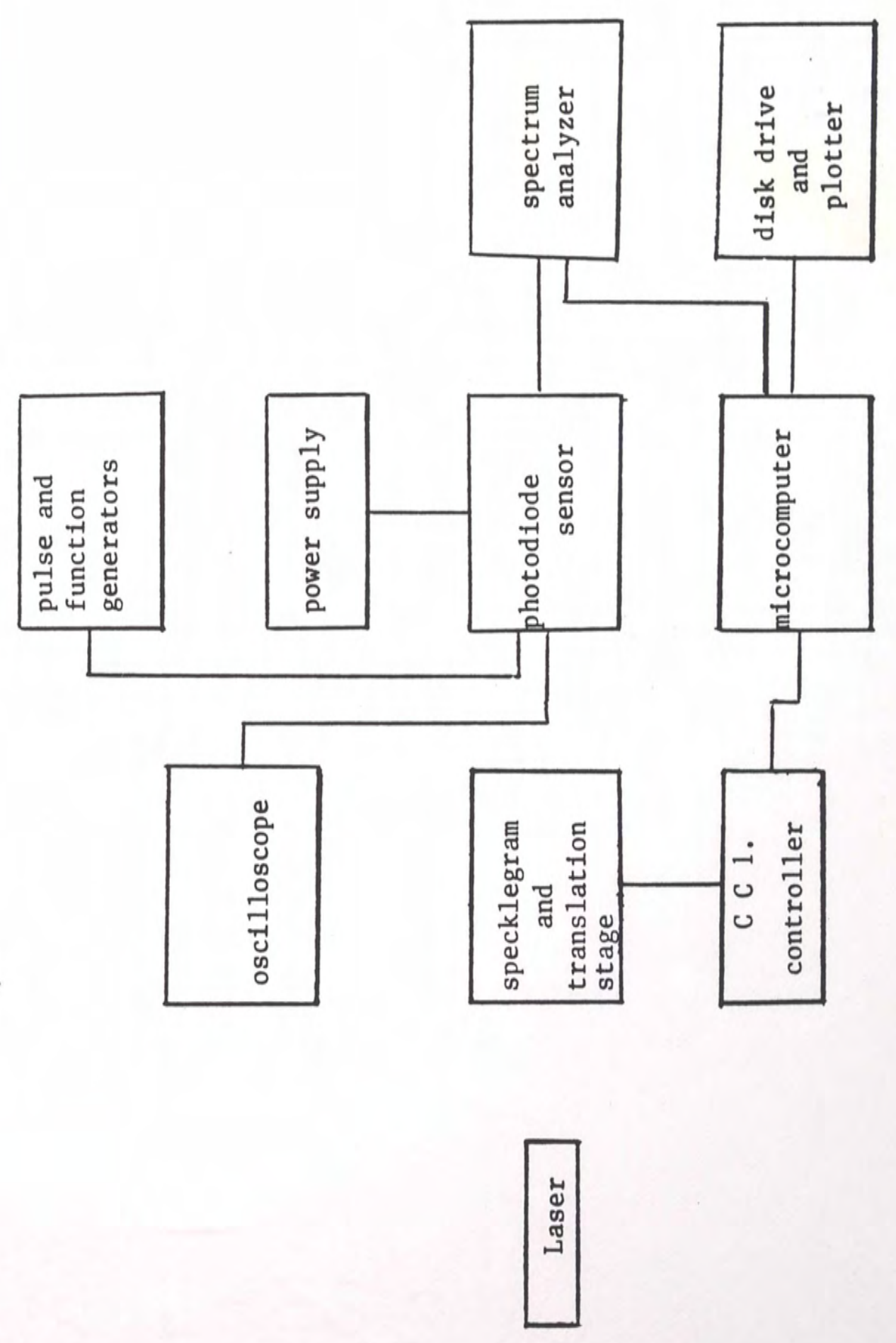

ปे 


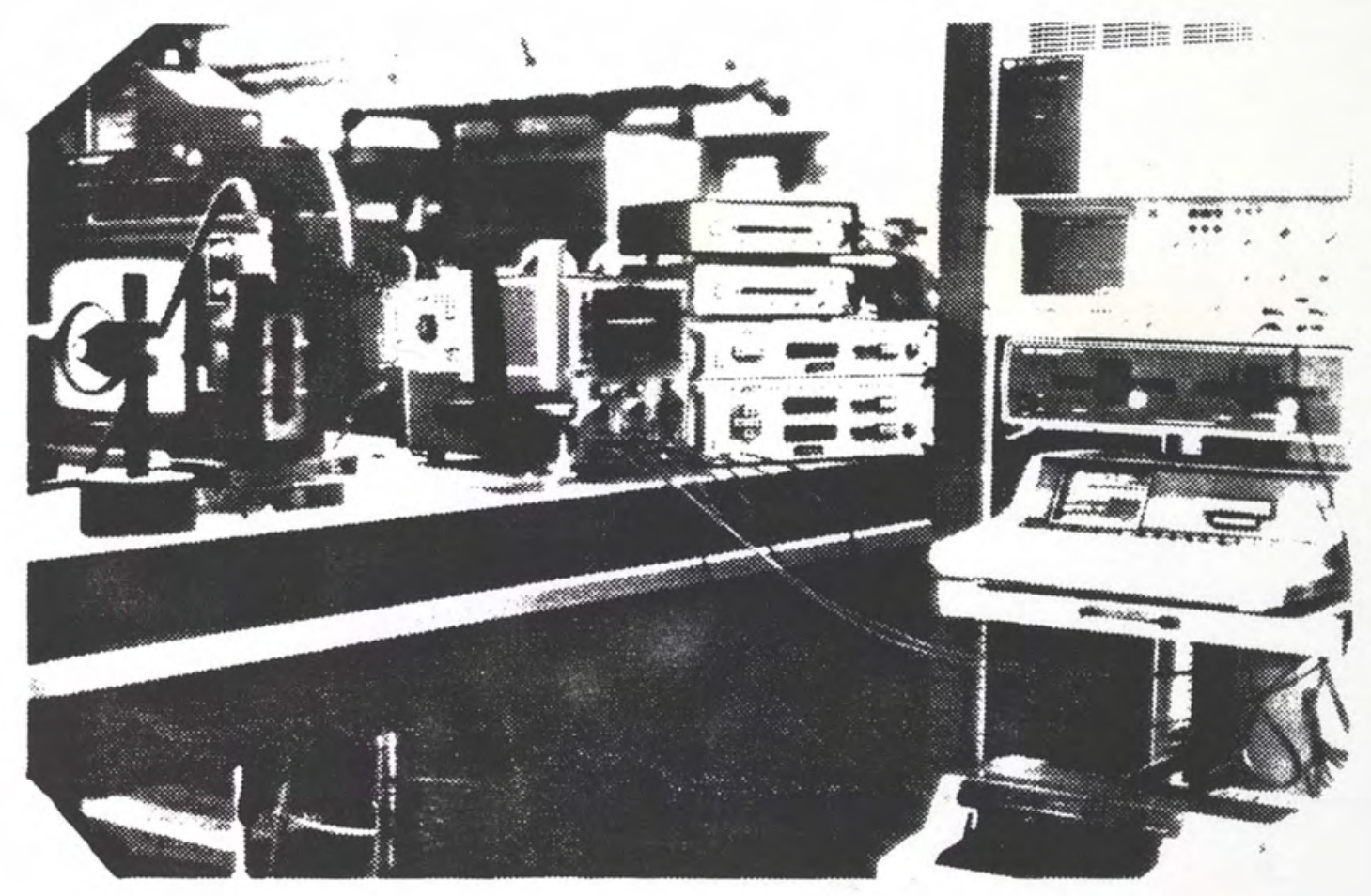

Figure 16. Photograph of the actual experimental set up. 


\section{Translation Stages}

The Klinger Scientific Corporation's translation stages are manual/programmable xyz axes with sensitivity range of 1-65700 micrometer. This type of translation stage was chosen since a high accuracy, as well as remote operation, was required. Remove operation of the stages was done via the HP-IB bus 1ine. The program, "STORDAT," was written in BASIC and was used to drive the stages.

\section{CCD-133D Photodiode Sensor}

Data was collected by Fairchild's CCD-133 photodiode array with 1024 pixels of $13 \times 13 \mathrm{microns}$ (Figure 17). This charge-coupled device results in total scan length of 13 millimeters. The photodiode sensor outputs data on two different lines. These data lines, namely $A$ and $B$, are bus lines which contain the signals from odd and even pixels. This device is operated on 20 volts and 0.3 amperes provided by Kepco Corporation's power supply model SC-32-2.5. Data collected by this array had to be expanded in order to be analyzed on the spectrum analyzer. The approach was to use a pulse generator and two signal generators. The pulse generator used was a Hewlett-Packard Model 222A, and the signal generators were Wavetek Model 182 .

\section{Oscilloscope}

Hewlett-Packard's Model 1201A oscilloscope was used to view time record data received from the photodiode sensor before filtering. 


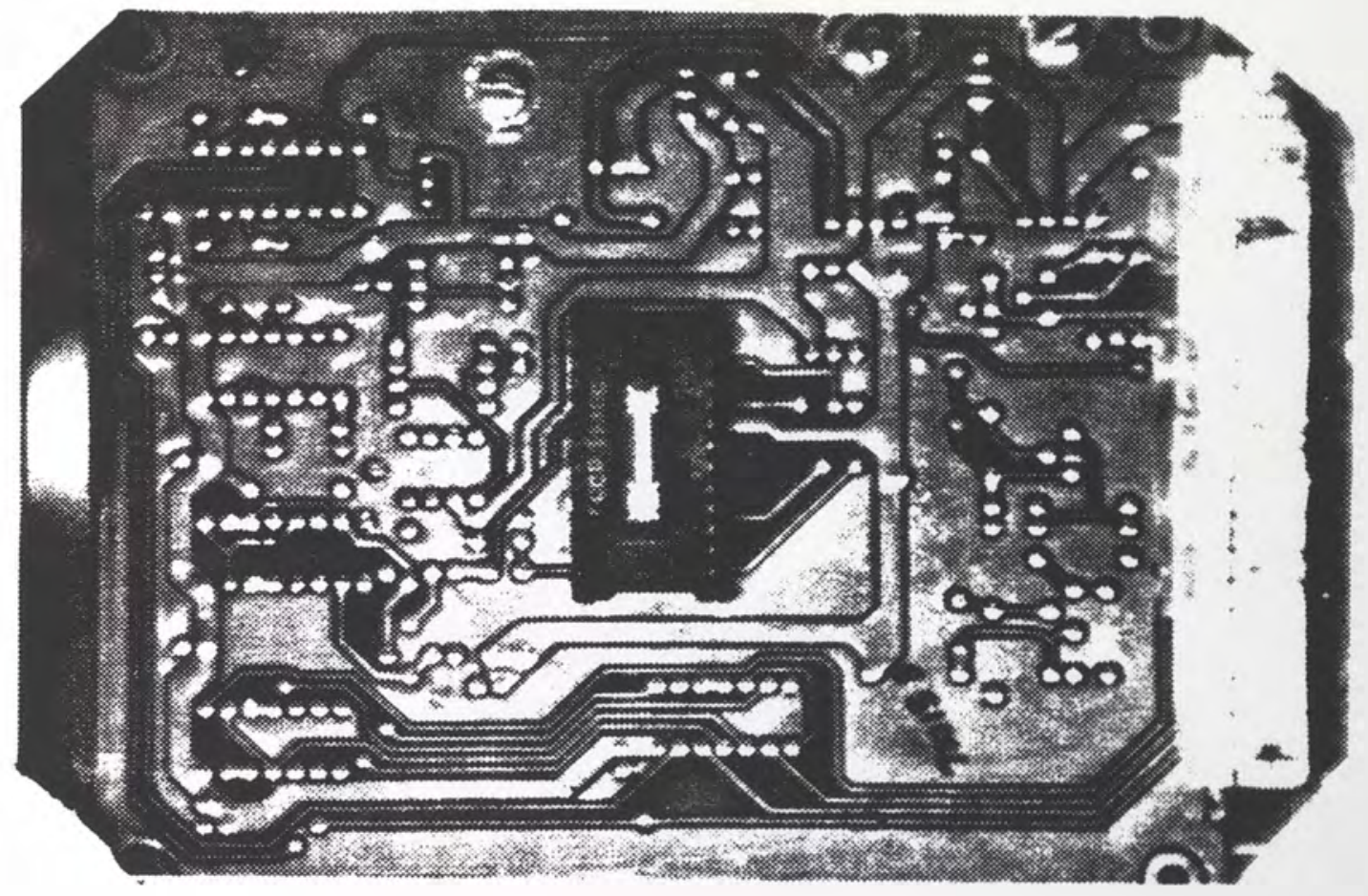

Figure 17. Photograph of CCD-133D, photodiode sensor. 


\section{Spect rum Analyzer}

Analog to digital conversion of raw data was possible utilizing the Hewlett-Packard Model 3582A spectrum analyzer. This device not only digitizes the data, but also performs the Fast Fourier Transform (FFT). Data is received from the sensor through the bus lines (A and B) connected to the front panel of the spectrum analyzer. Data is stored in the memory buffer of the analyzer for further analysis. The memory buffer is accessed by the front panel switches or by remote operation through a microcomputer. The HP-3582A spectrum analyzer has three passband shapes available: hanning, uniform and flat top. Each one is best used for different tasks. For example, hanning is used when frequency accuracy is required; uniform is used when maximum frequency is of importance; and flat top is used when best amplitude accuracy is required. For this project, the hanning passband shape was most suitable.

\section{HP-85 Microcomputer}

Data collection and storage, remote control of the translation stages and the spectrum analyzer were possible by the Hewlett-Packard Model 85 Microcomputer. Due to the low storage capacity (about 30K byte) of the HP-85, a Hewlett-Packard Model 82901M flexible disk drive was used. This device provided enough storage area to manipulate and store data on a floppy disk. To plot the stored data or the current data, a Hewlett-Packard Model 7470A plotter was used. 


\section{CHAPTER V}

\section{RESULTS AND DISCUSSION}

In order to test and calibrate the CCD-133 photodiode sensor, artificial fringes having different spacing were produced on transparencies as shown in Figure 18. A fringe transparency is placed in the film plate holder and illuminated by white light from a slide projector. Fringes are imaged on the sensor using a lens with magnification ratio $M=1.54$ (Figure 19). The previous procedure was repeated six times for different fringe spacing sets.

Time record data on the spectrum analyzer appeared to be sinusoidal. These data were Fourier transformed. Figures 20 through 25 represent the time record data, and its corresponding Fourier transform plots, respectively, for the six different cases. As shown in FFT plots, the maximum peak occurs at the fundamental frequency of these waves and is marked with an asterisk. The resultant frequencies were then inverted, tabulated and plotted in Table 1 and Figure 26 against the fringe spacing seen by the sensor. Figure 26 shows that inverse of frequency and fringe spacing has a linear relationship, and by a least squares method, it was found that this relationship had an equation $d^{\prime}=46.63 \cdot 1 / f+0.00319$, where $f$ is the frequency in $\mathrm{Hz}$, and $\mathrm{d}^{\prime}$ is the fringe spacing at the plane of sensor in inches. The correlation coefficient for this linear relation was 0.99. This means that if the frequency is $400 \mathrm{~Hz}$, the corresponding fringe spacing will be approximately 0.12 inch. 


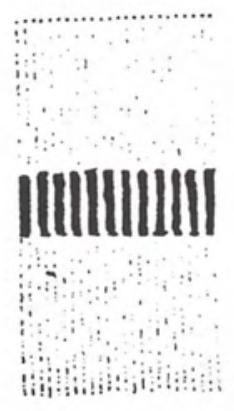

0.03 in. separation

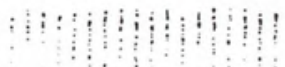

||l|||||

0.05 in. separation

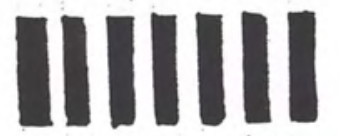

0.075 in. separation

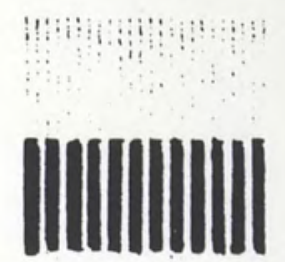

0.042 in. separation

0.0583 in. separation

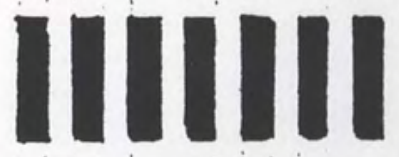

0.10 in. separation

Figure 18. Artificial fringes and their respective spacing used in the experiment. 


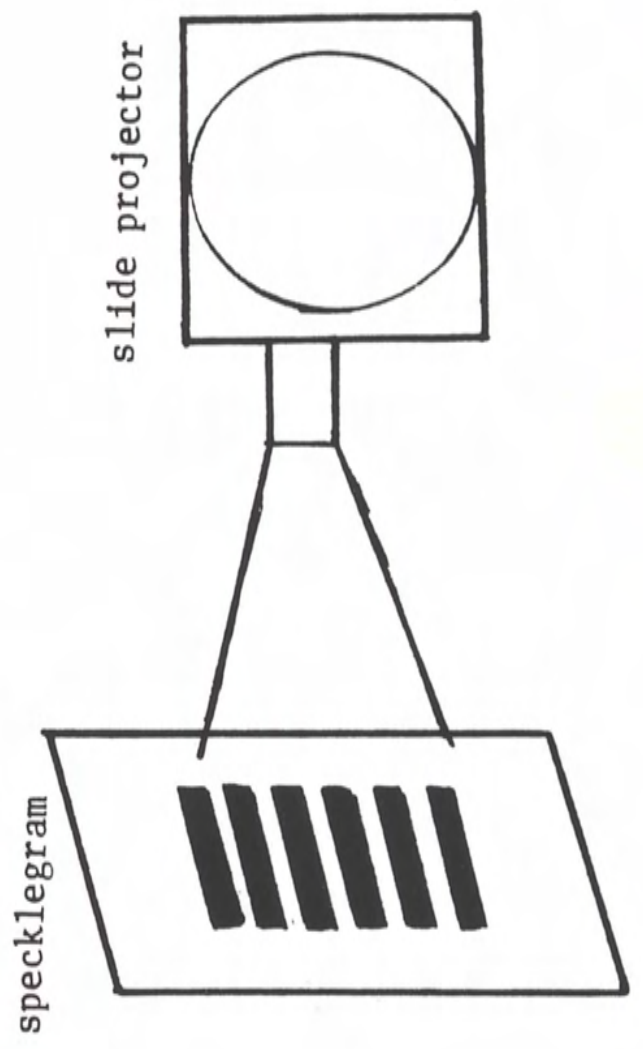

อั

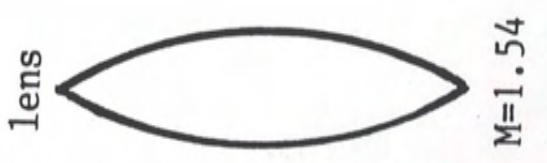

齐

党

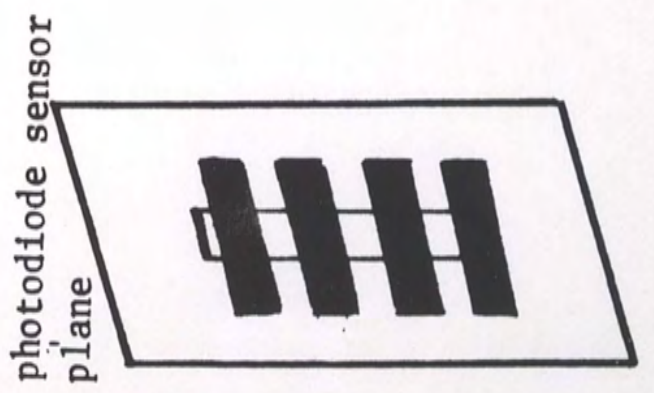

吾

a

旁 


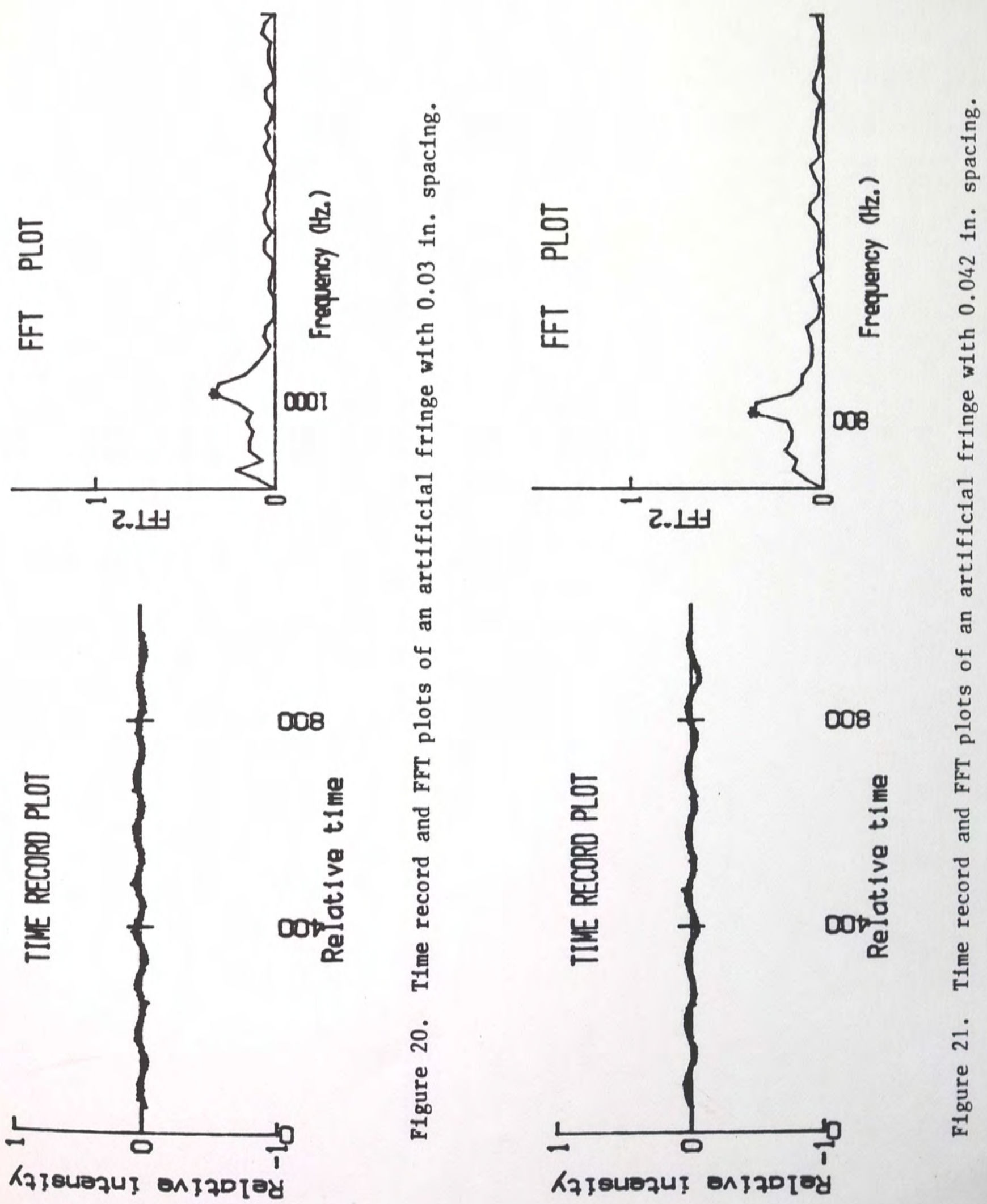




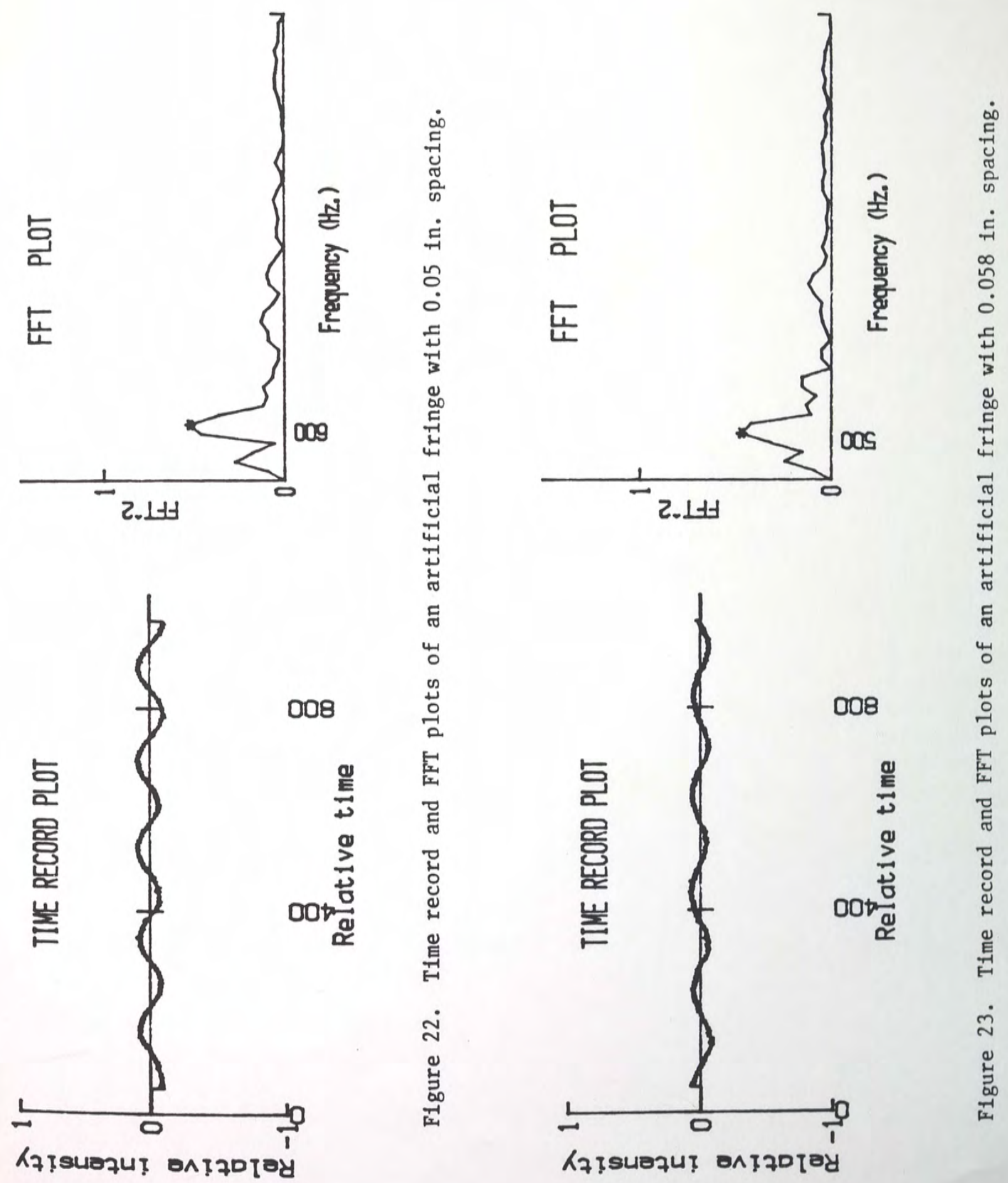



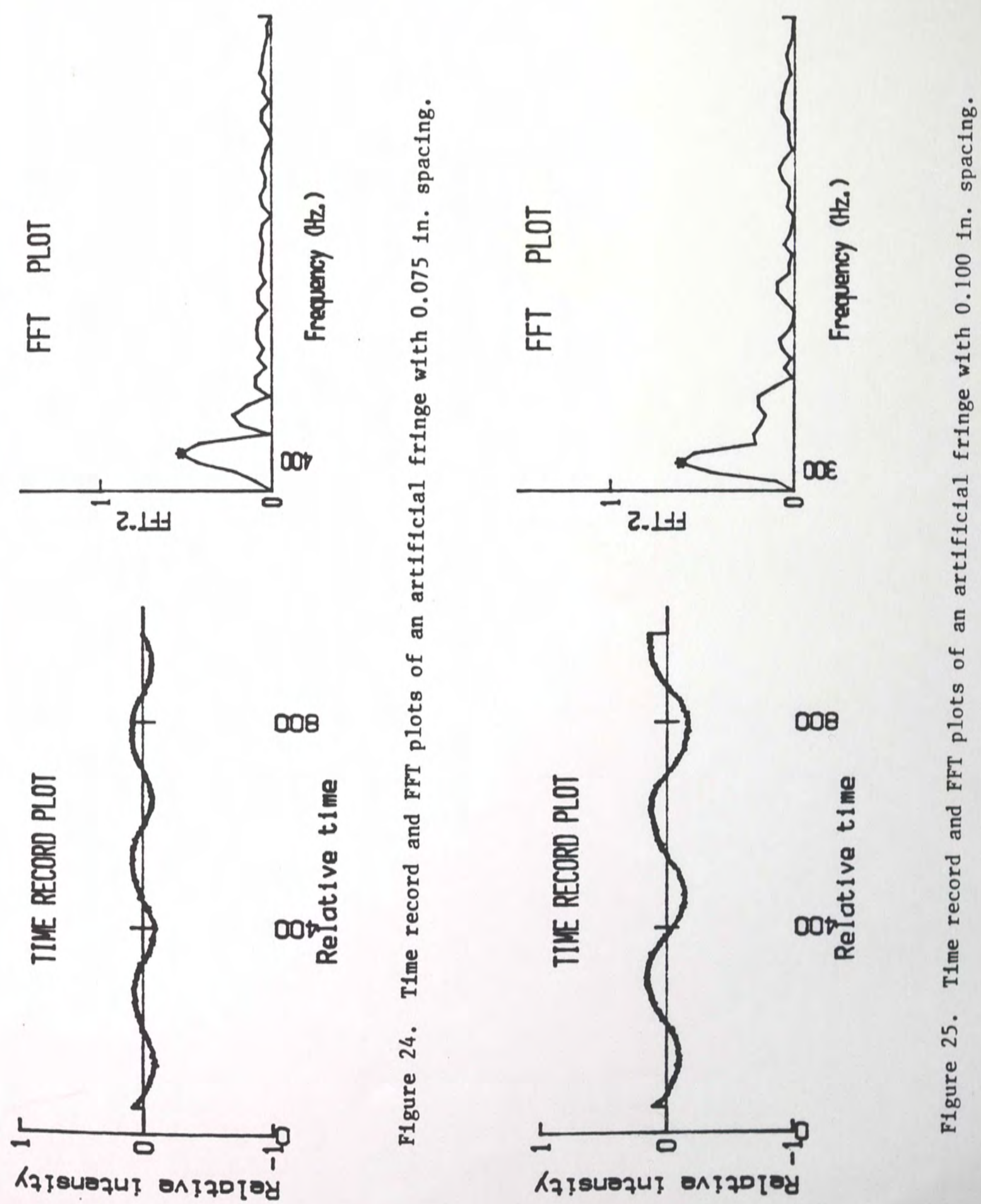

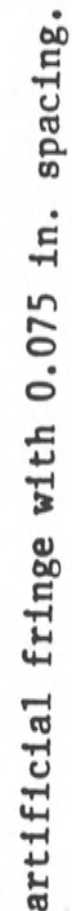

年

$-1$

동

茨

品

点

뮤

엉

兽

ஸ்

峁

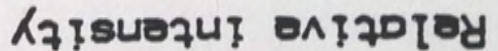




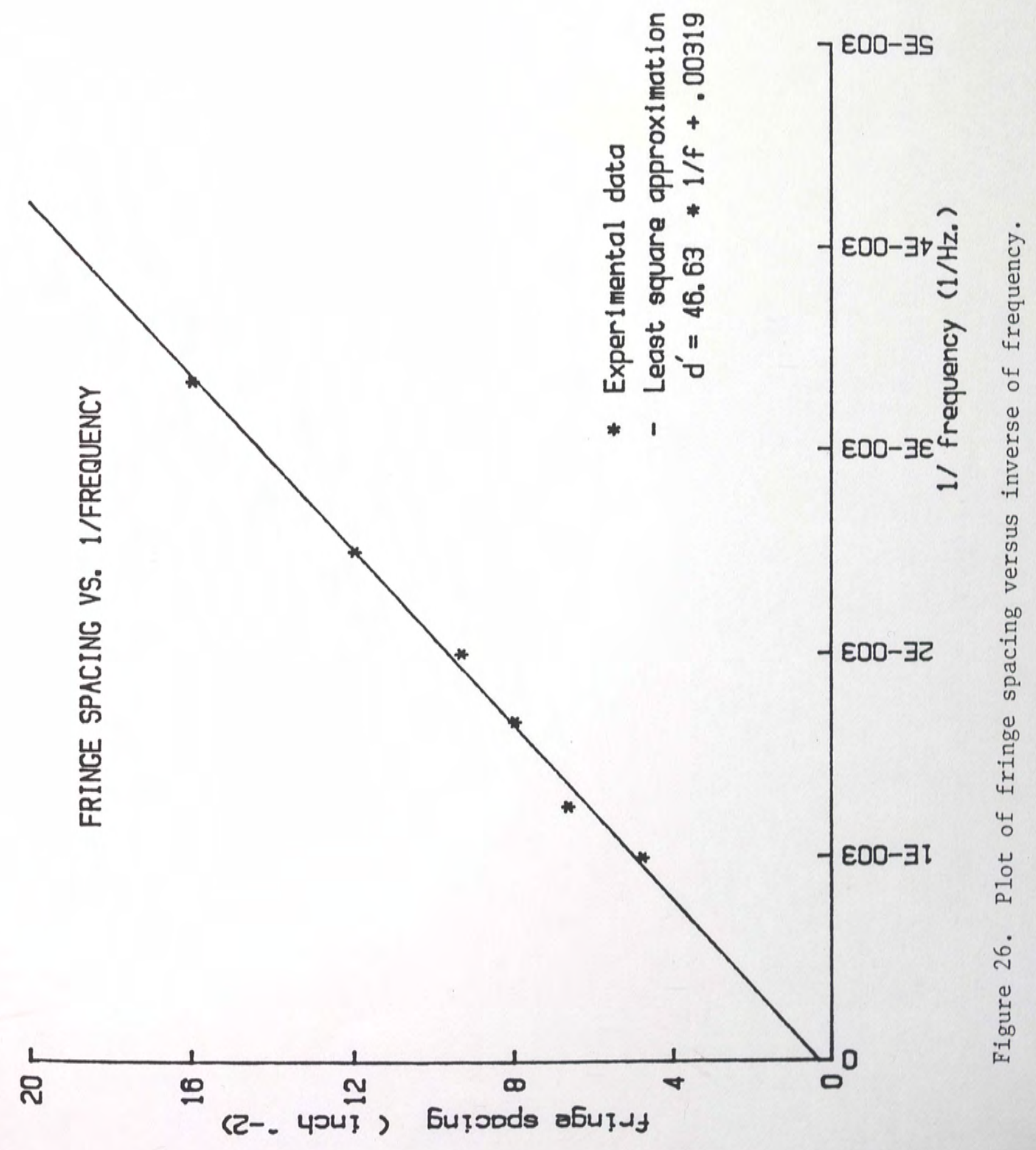


TABLE 1

FRINGE SPACING VERSUS INVERSE OF FREQUENCY

\begin{tabular}{c|c}
\hline $\begin{array}{c}\text { FRINGE SPACING } \\
\text { (in) }\end{array}$ & $\begin{array}{c}\text { INVERSE OF FREQUENCY } \\
(1 / \mathrm{Hz})\end{array}$ \\
\hline 0.0479 & 0.0010 \\
0.0666 & 0.0013 \\
0.0798 & 0.0017 \\
0.0932 & 0.0020 \\
0.1198 & 0.0025 \\
0.1598 & 0.0033 \\
\hline
\end{tabular}

After the calibration of the sensor, a specklegram of a $1 / 4$ inch thick cantilever beam subjected to transverse-end loading was produced. Material for the beam was plexiglass with $\mathrm{E}=4.56 \times 10^{5}$ psi and $\nu=0.3$. The transverse-end load was governed by displacement of the free end, which was 0.005 inch. Figure 27 shows the experimental set-up for recording the double-exposure specklegram. Dimensions of the beam were 5 inches in length and 1 inch in width, with an imaging magnification factor of 0.75 . The produced specklegram was then illuminated with an unexpanded He-Ne laser beam. The laser beam was passed through a point on the centerline of the beam resulting in a circular halo modulated by Young's fringes on the plane of the sensor. 


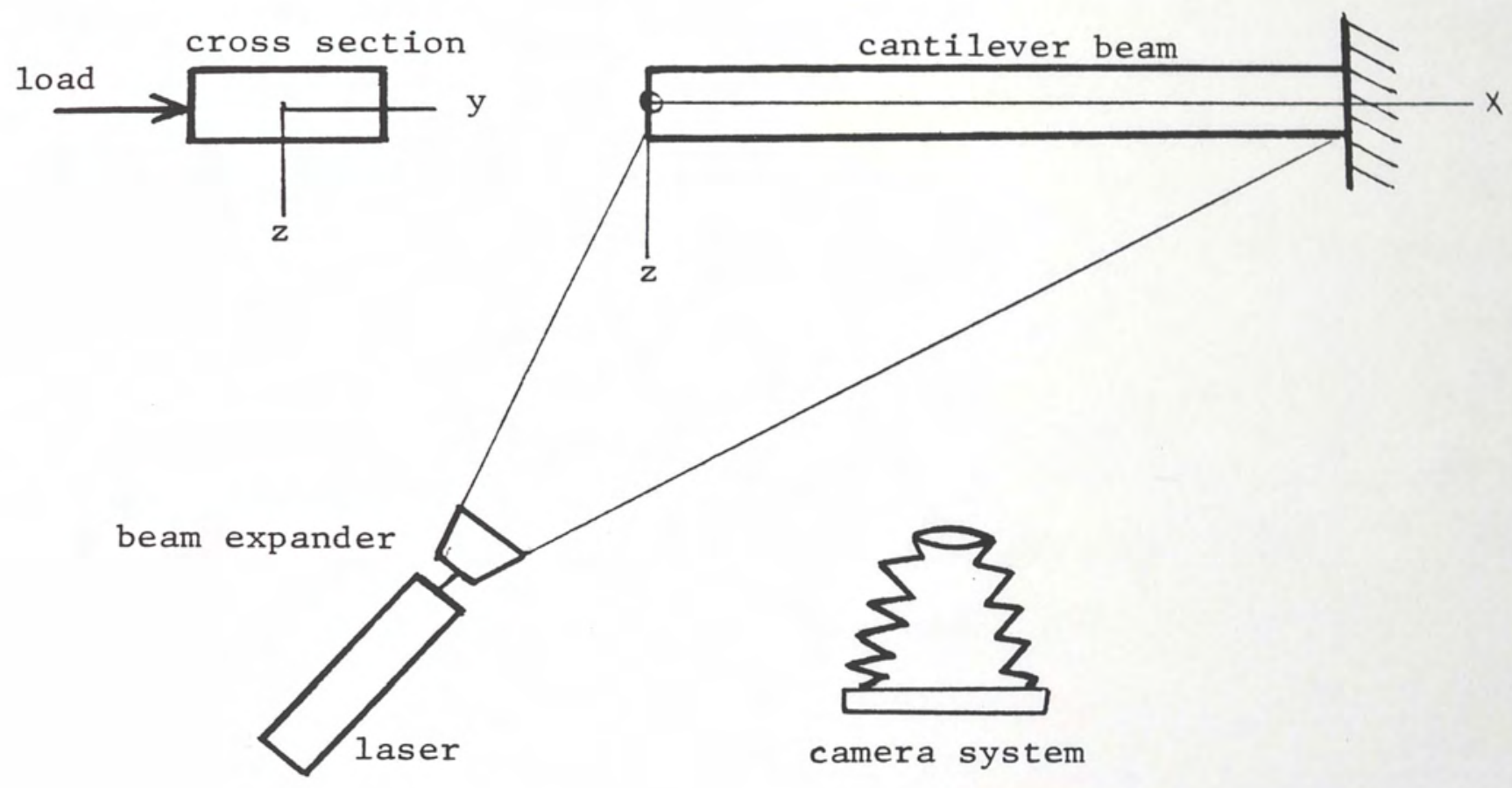

Figure 27. Experimental set up for producing double-exposure specklegram of the cantilever beam experiment. 
Scanning along the beam centerline was selected since the displacement of any point along the centerline is vertical and this will produce horizontal fringes perpendicular to the sensor orientation. Measurements of displacement of other points was not attempted here since it would be necessary to reorient the sensor.

The specklegram was then displaced using the computer-controlled translation stages until three fringes were observed at the plane of the sensor. The fringe pattern was digitized along a line perpendicular to it using the CCD sensor. This resulted in a record of relative light intensity versus distance, which was converted to a time record by the spectrum analyzer. The time record is stored in the computer and is shown in Figure 28. The noise observed in the time record of the intensity data is due to the speckle effect on the halo. A fourier transform was performed on the time record of the data using the spectrum analyzer and the fundamental frequency (corresponding to the location of the maximum peak) was found to occur at $300 \mathrm{~Hz}$, as shown in Figure 28.

Furthermore, scanning along the centerline of the beam was conducted as controlled by the computer until 4, 5, 6 and 7 fringes were observed. Their time record and corresponding FFT records were plotted in figures 29 through 32 .

Table 2 represents the tabulated data for number of fringes versus corresponding frequency in hertz. This relationship was then plotted and approximated by a straight line having the equation 

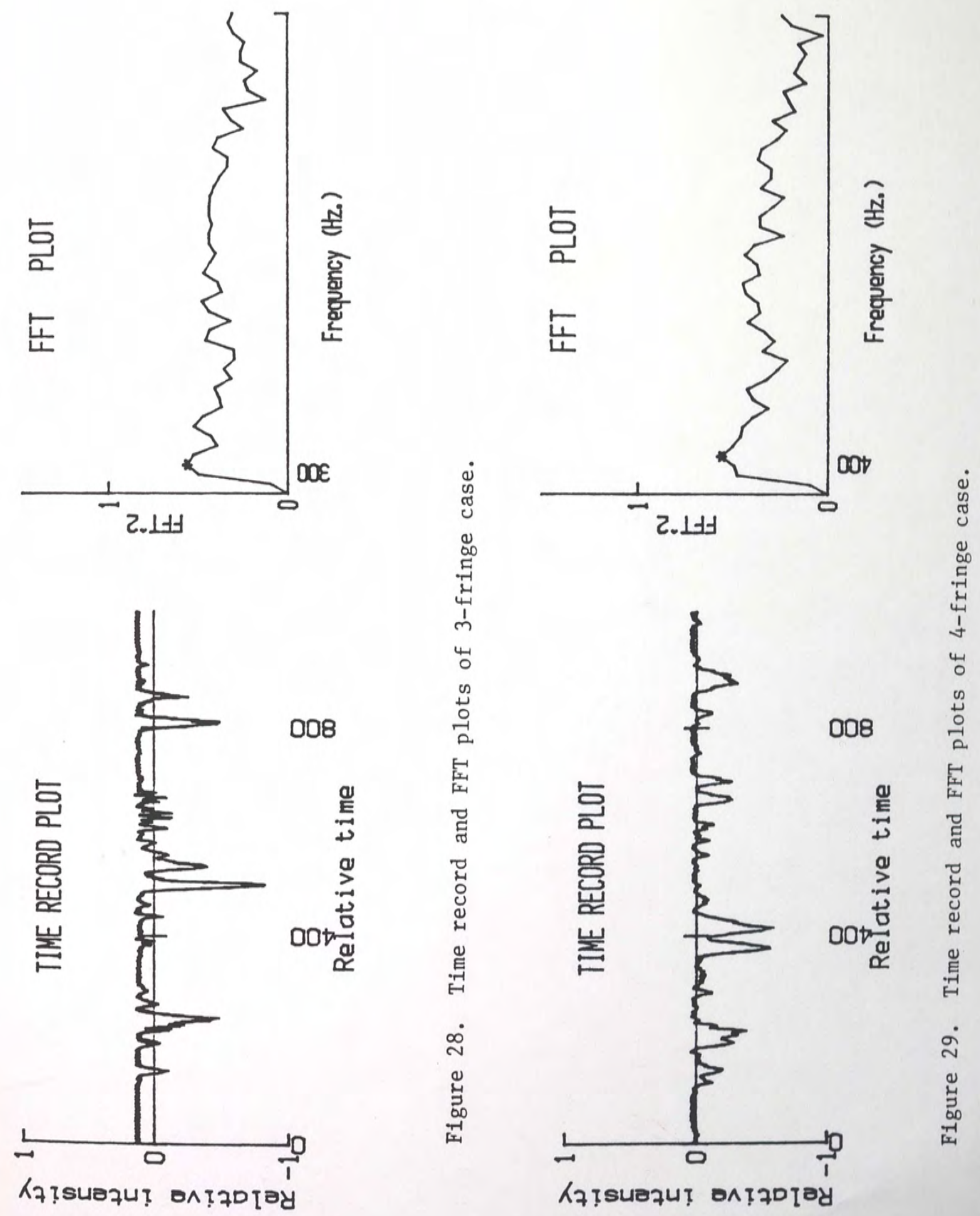

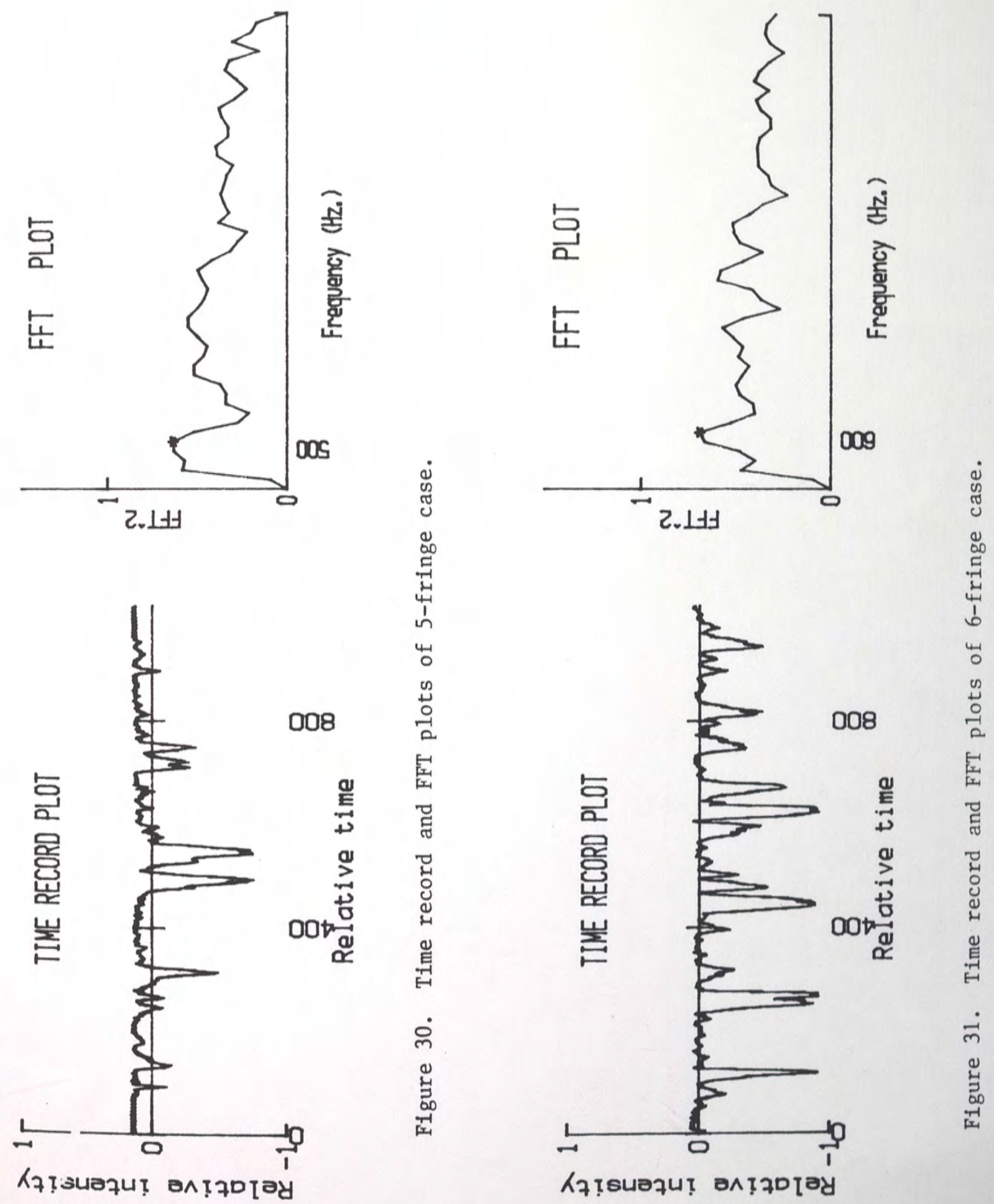


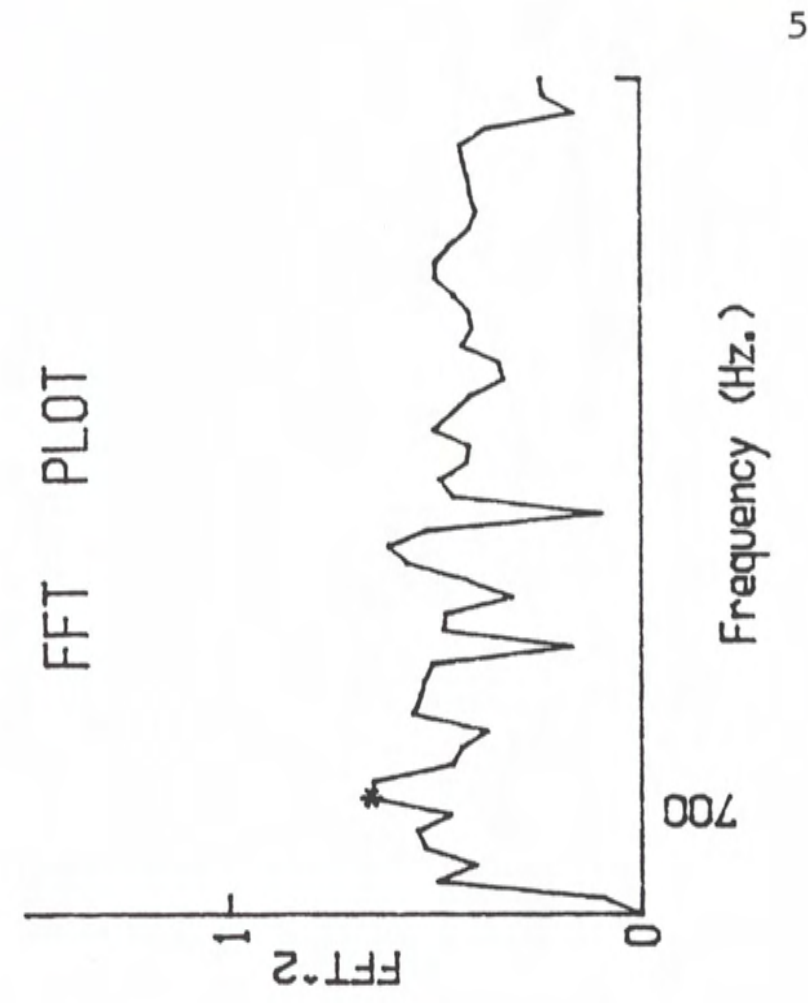

50

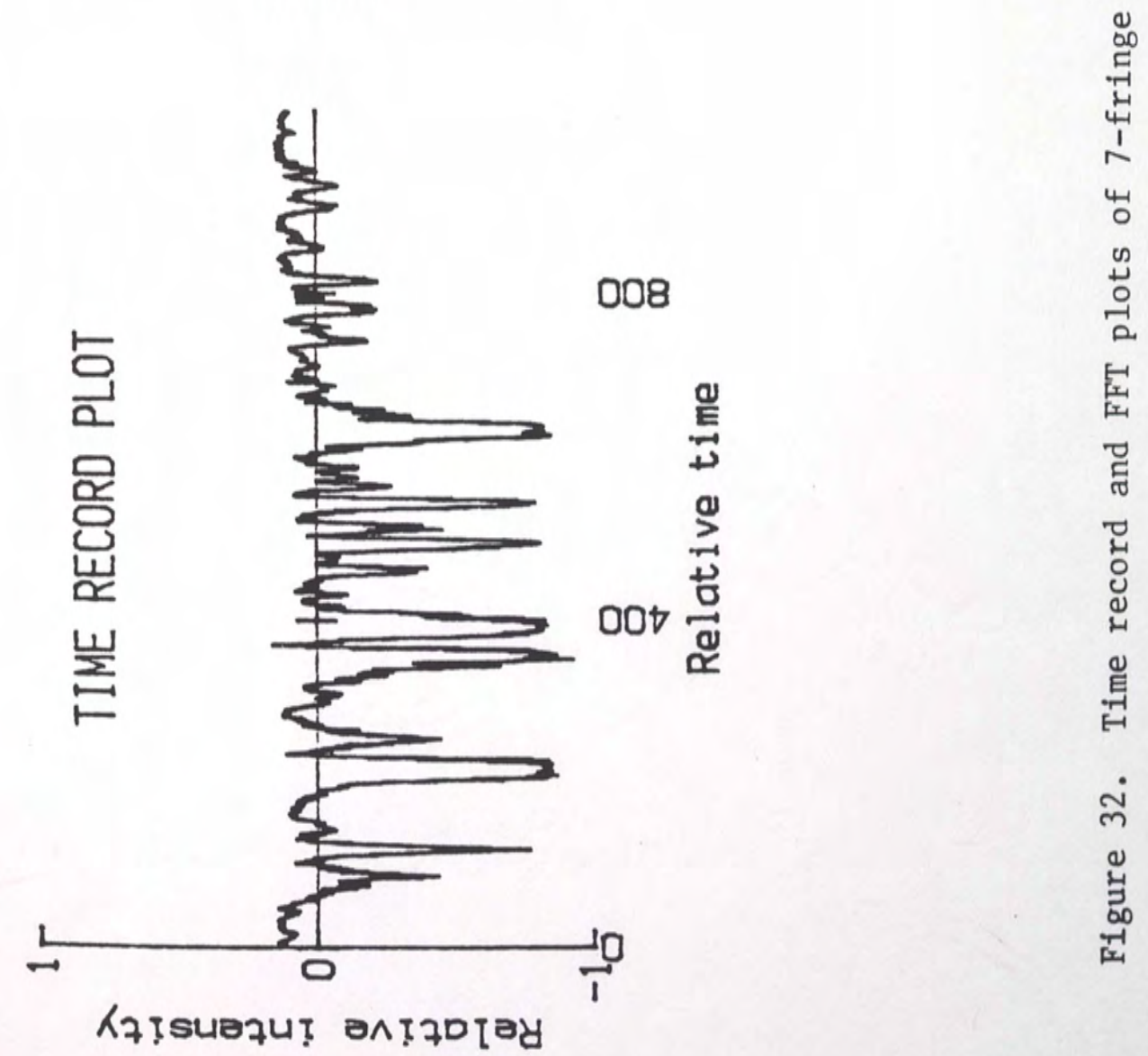


$N=0.01 \cdot f$, with correlation coefficient equal to 0.99 , as shown in Figure 33.

TABLE 2

FRINGE NUMBER VERSUS FREQUENCY

\begin{tabular}{c|c}
\hline FRINGE NUMBER & $\begin{array}{c}\text { FREQUENCY } \\
(\mathrm{Hz})\end{array}$ \\
\hline 3 & 300 \\
4 & 400 \\
5 & 500 \\
6 & 600 \\
7 & 700 \\
\hline
\end{tabular}

The previous data was collected from any single scan in a direction perpendicular to the fringes. This data may not include all the information. Therefore, the following experiment was conducted. The photodiode sensor was displaced, four times to the right of the bright spot on the halo and four times to the left of the bright spot. The case of three fringes was chosen for this experiment. Scans were separated by $0.001 \mathrm{inch}$, as shown in Figure 34 . Time record plots and corresponding FFT plots for four scans to the right of bright spot are shown in figures 35 through 38 . Figures 39 through 42 represent the time record plots and corresponding FFT plots for four scans to the left of the bright spot. The average of 


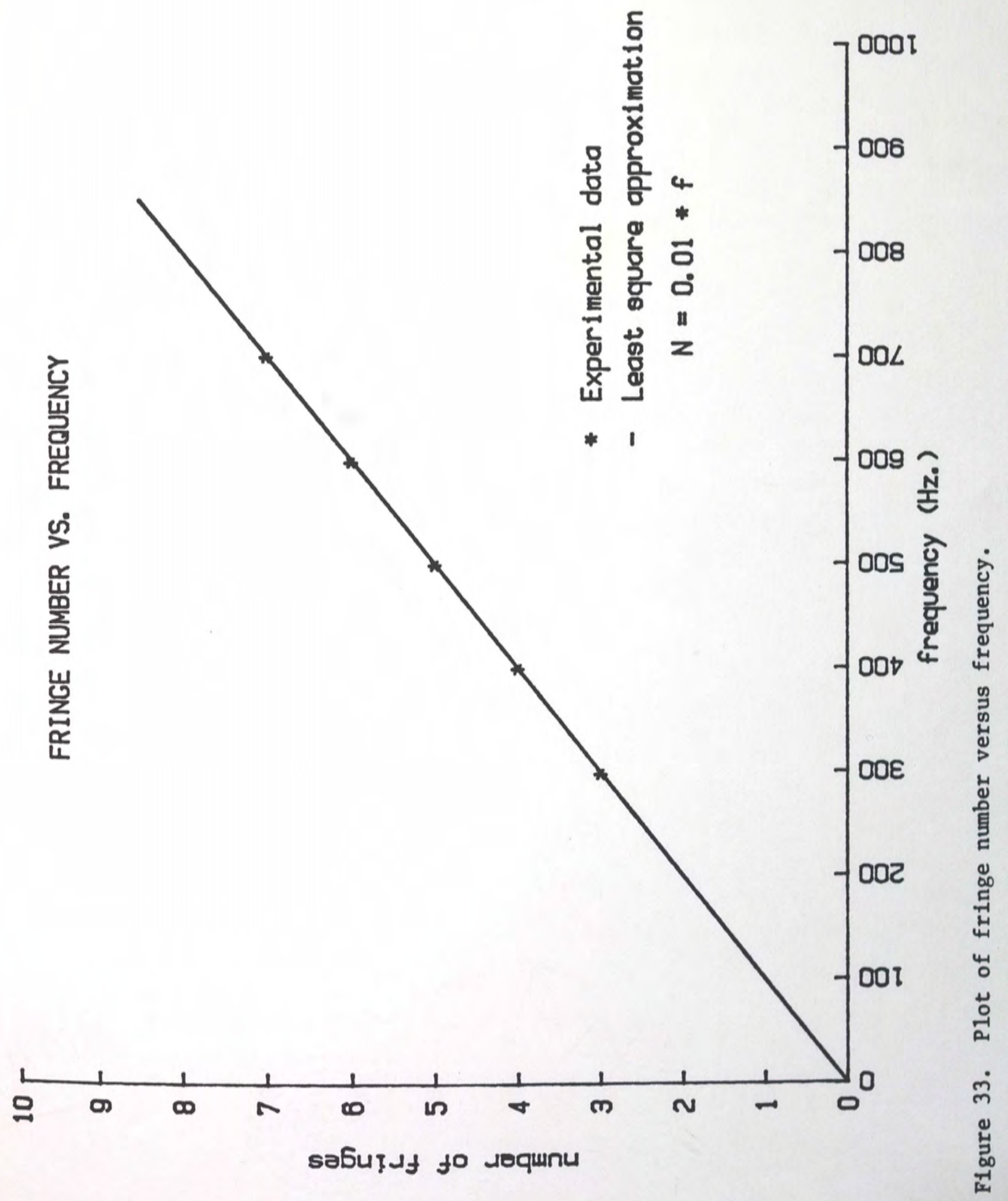




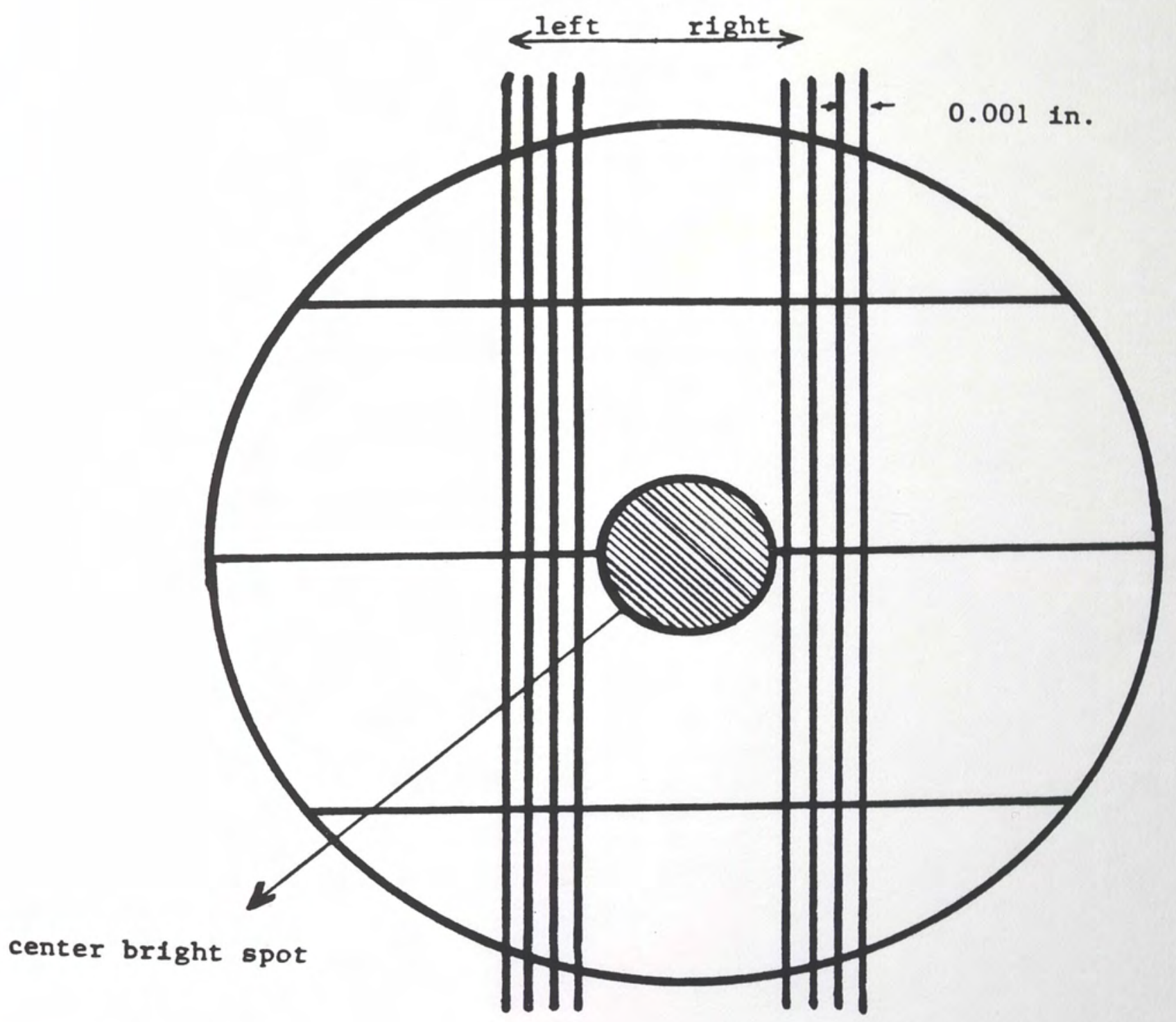

Figure 34. Scan locations on the fringe halo. 

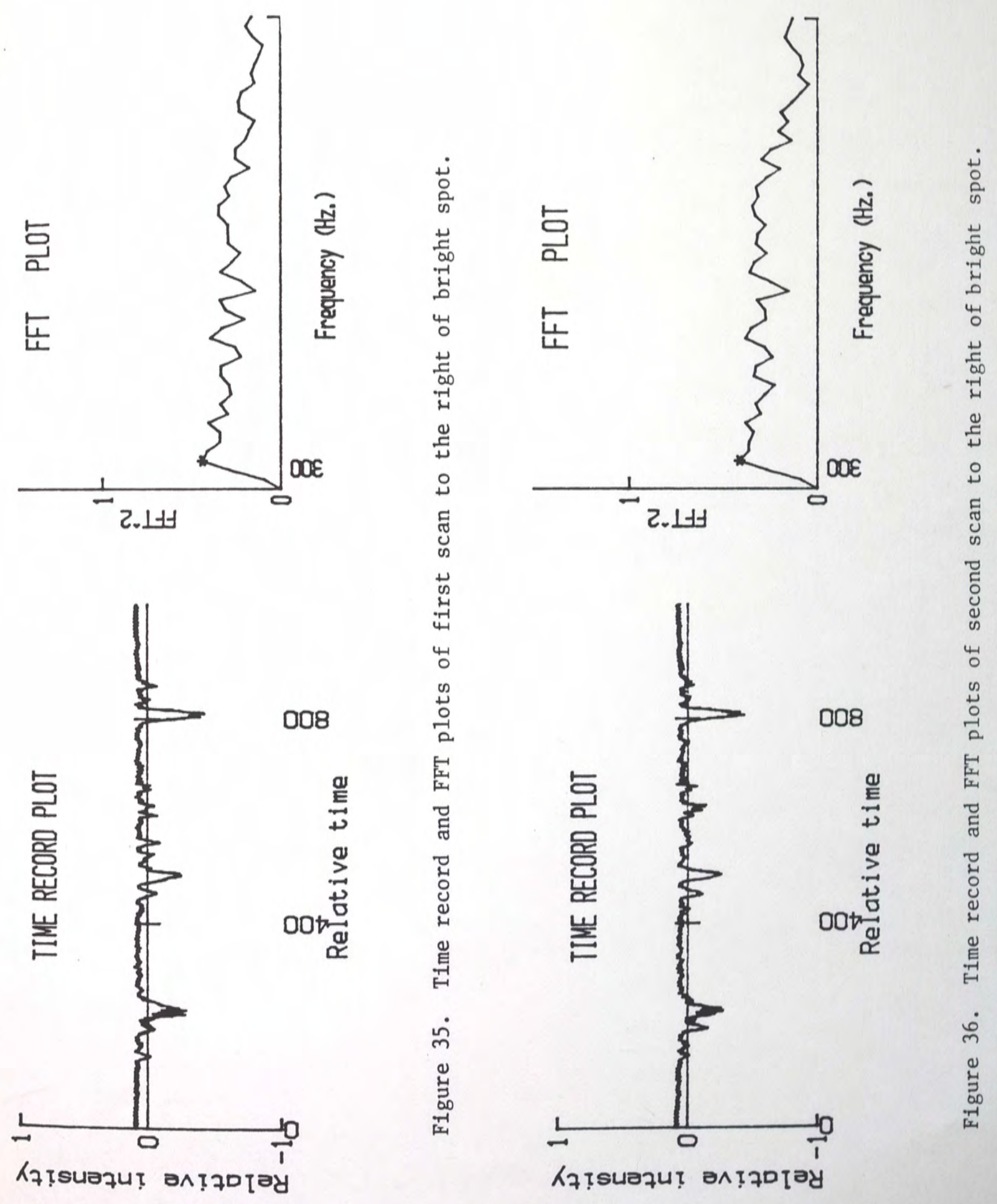

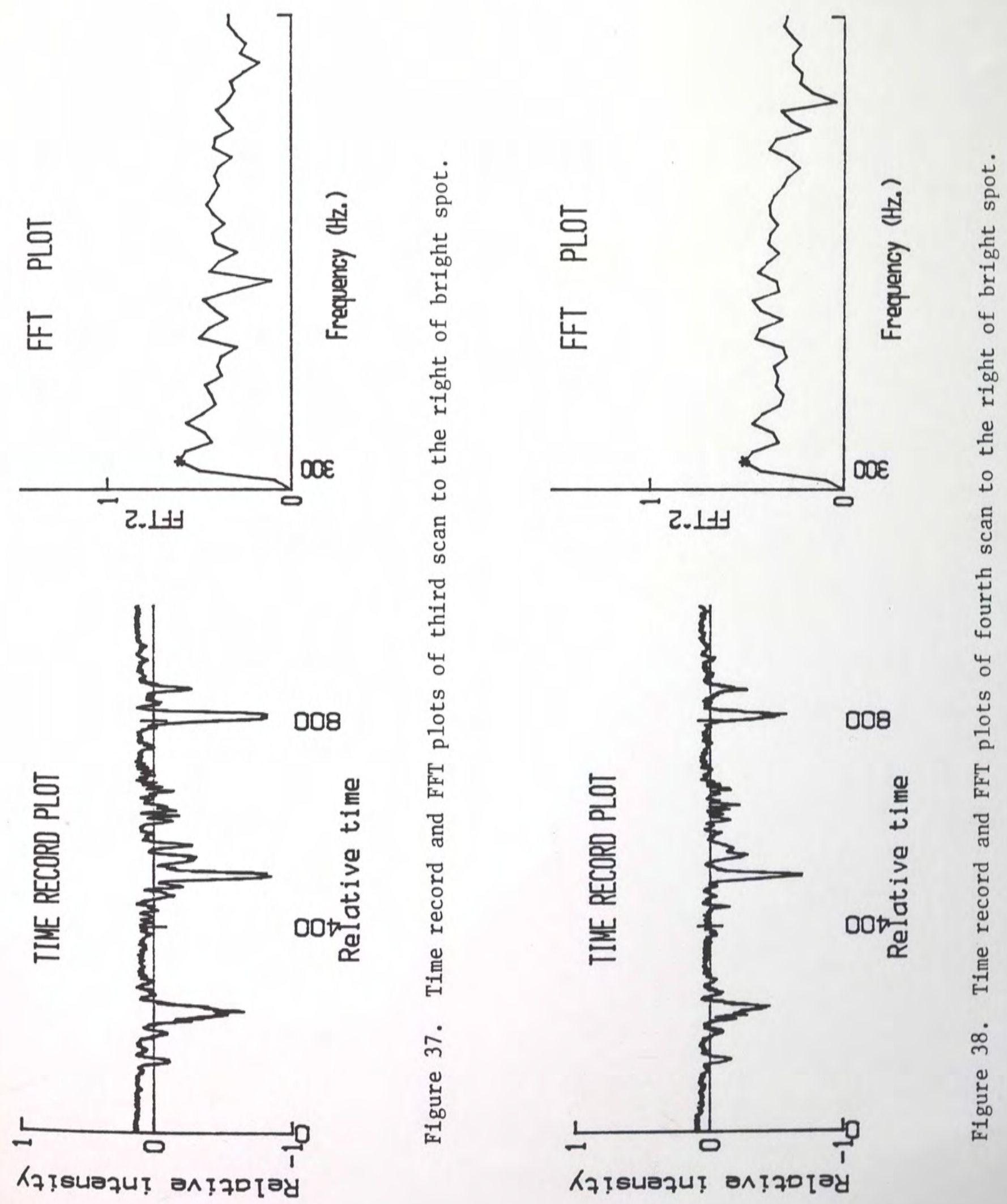

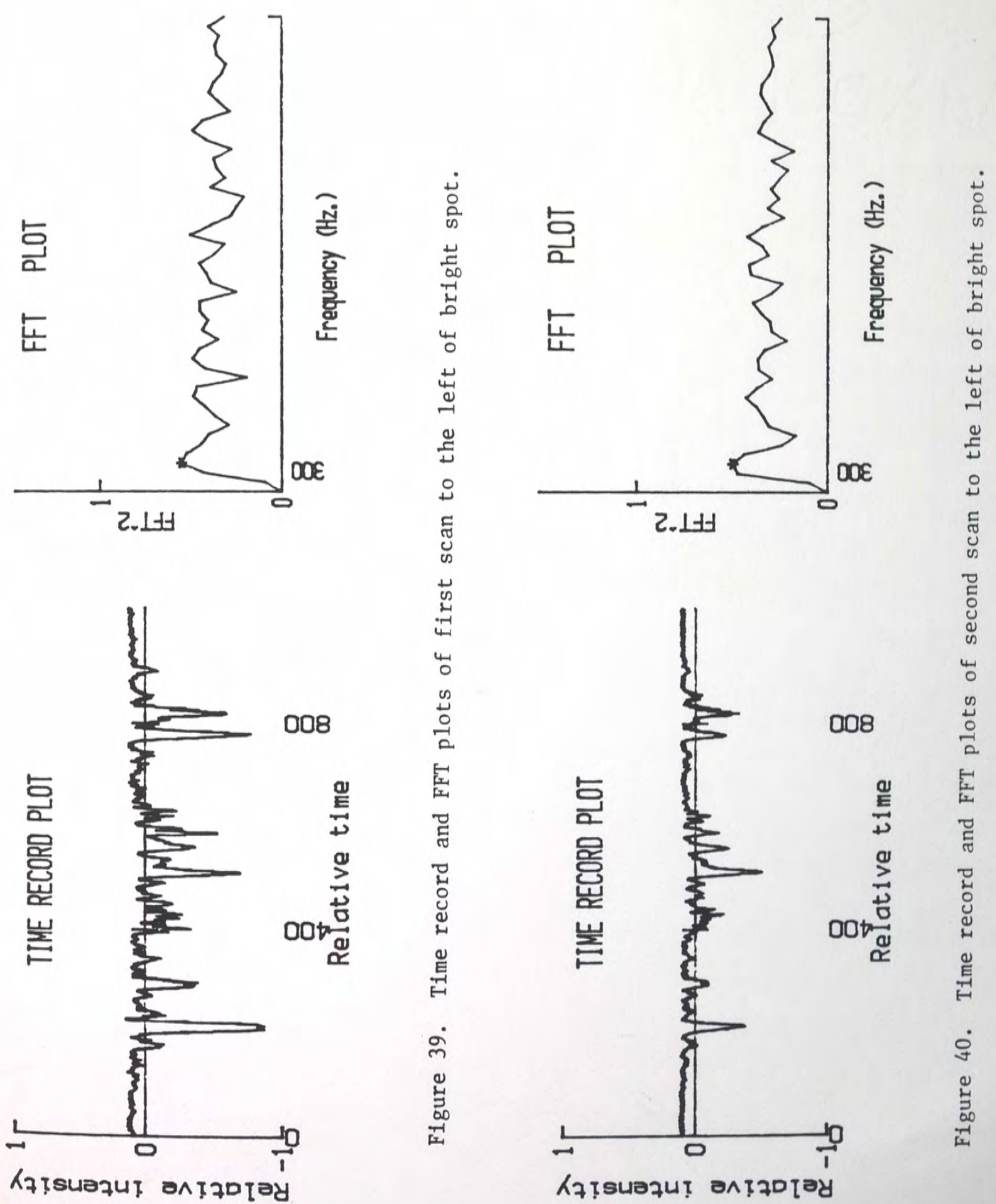

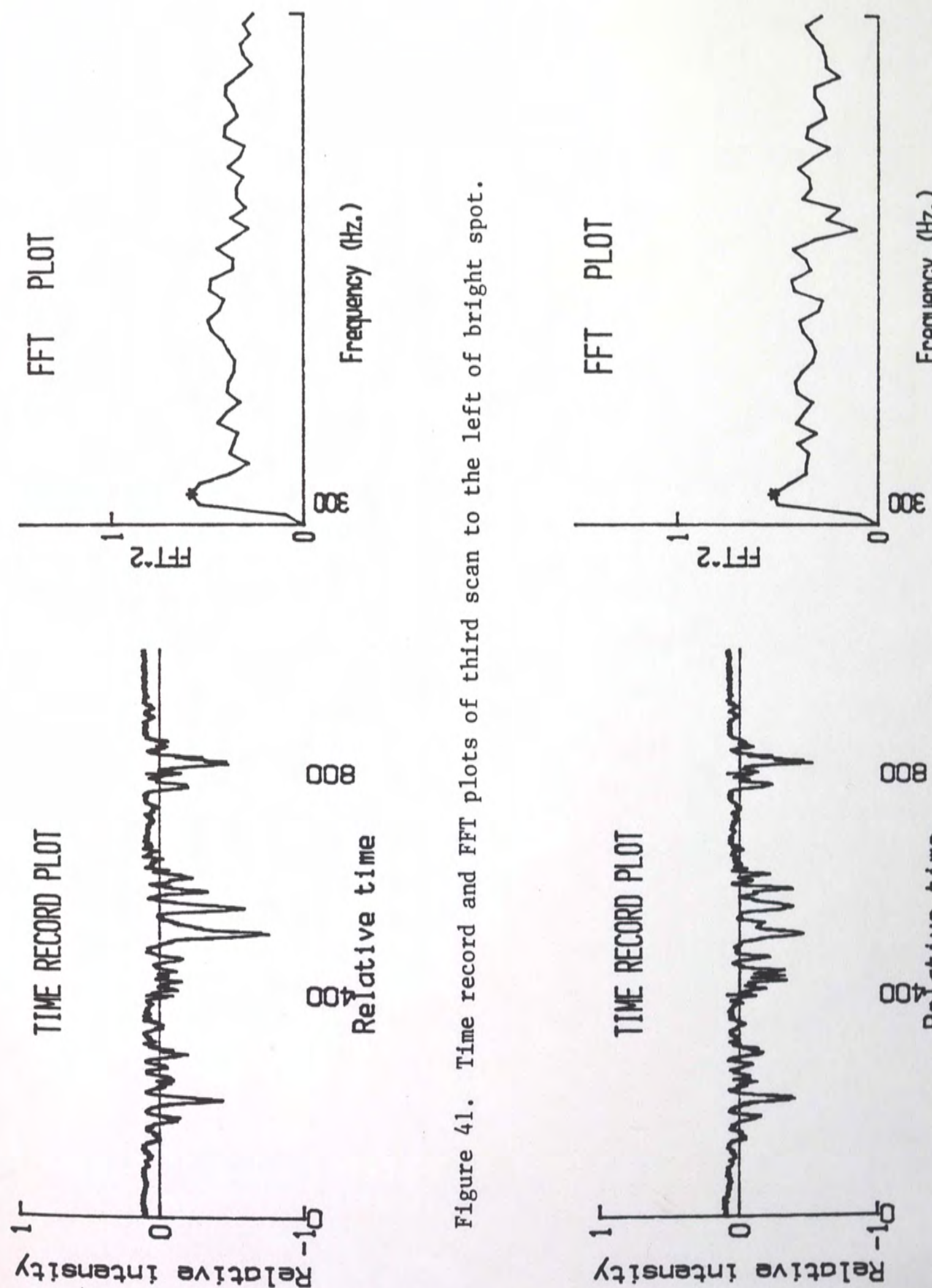

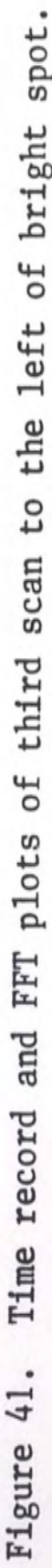

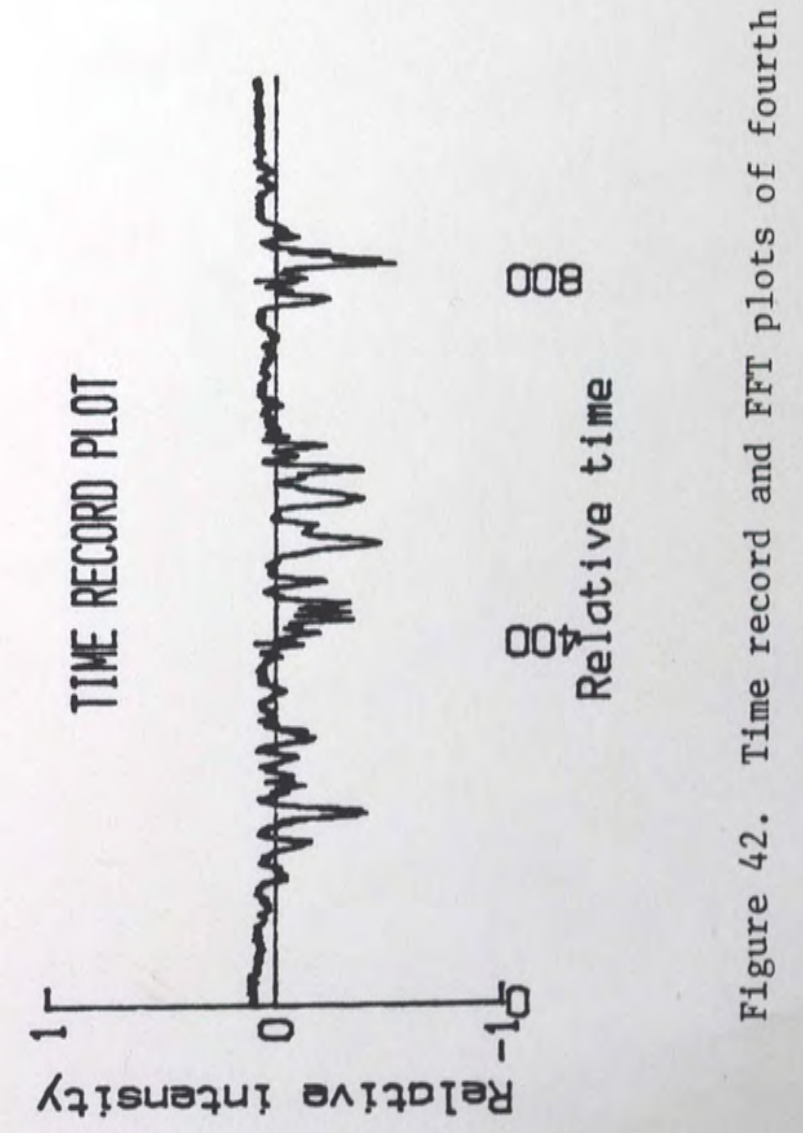


the square of the FFT plots was computed and plotted in Figure 43. As can be seen, the effect of the averaging process has smoothed the FFT plot, but has not affected the location of the peak. This simply was due to the limited resolution of the FFT analyzer in detecting the fundamental frequency. Therefore, it was decided that one scan would be sufficient and will be used in the rest of the experiment ation.

Finally, the double-exposure specklegram of the cantilever beam was illuminated again at different points (controlled by the translation stages and the computer) along the beam neutral axis to measure the vertical displacement at various locations. Figure 44 shows the cantilever beam with the sample scan locations separated by 0.26 inch. In this experiment, a camera lens with 8.25 inch focal length was used to image the fringe halo on the plane of the sensor from the viewing plane (ground glass). The fringe size on the viewing plane was smaller than on the sensor plane. The magnification factor used was $M^{\prime}=1.2$. The experimental set-up is shown in Figure 45. Fringe images were then digitized and analyzed one at a time by the spectrum analyzer and the computer. Time record and FFT plots for these sample points are presented in figures 46 through 65 . The fundamental frequency obtained from FFT plot was then used to calculate the fringe spacing utilizing the relationship obtained for the fringe spacing, $d^{\prime}$, and the inverse of frequency, $1 / f$ :

$$
d^{\prime}=46.63 \cdot 1 / f+0.00319
$$




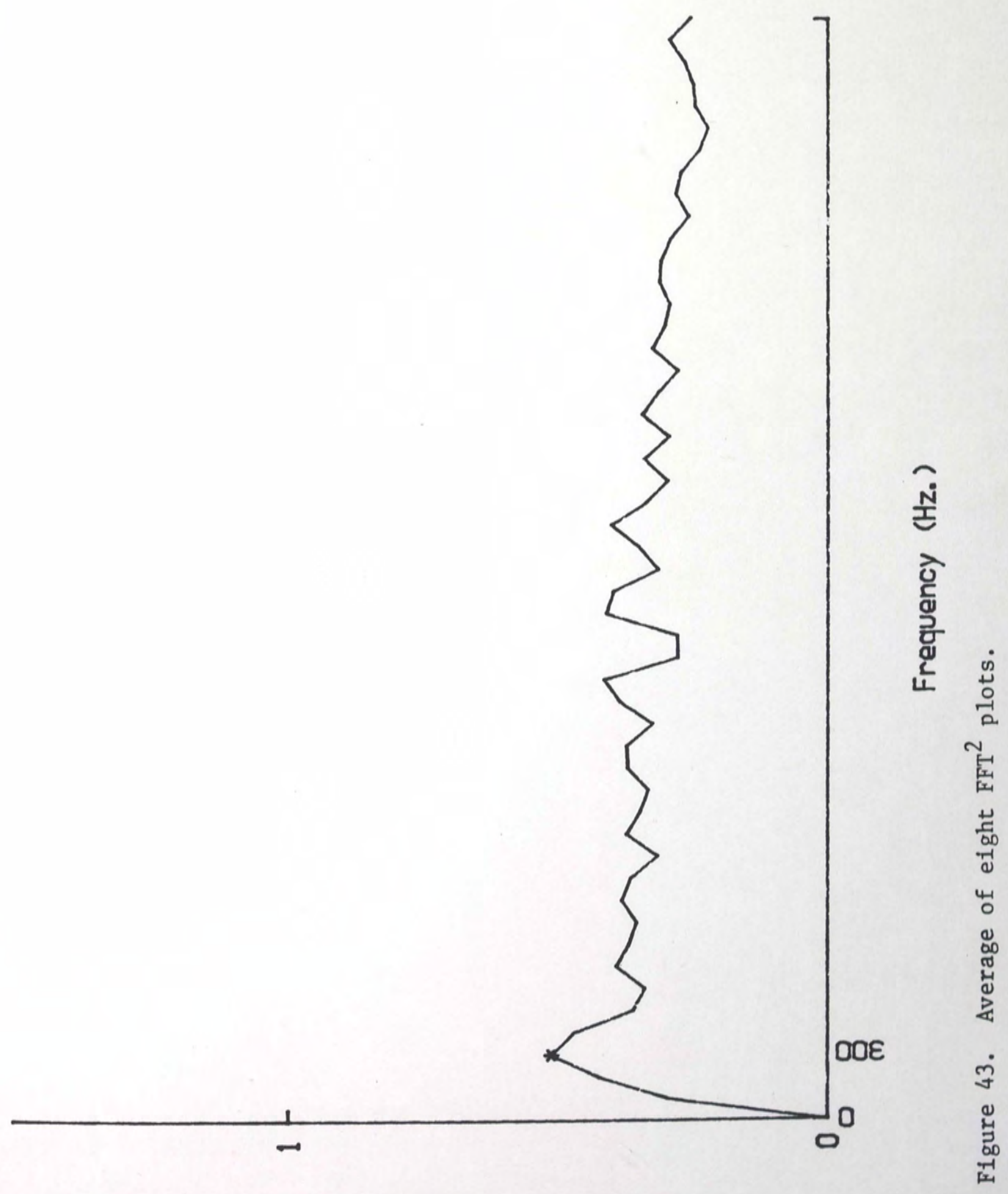

2.1 19 


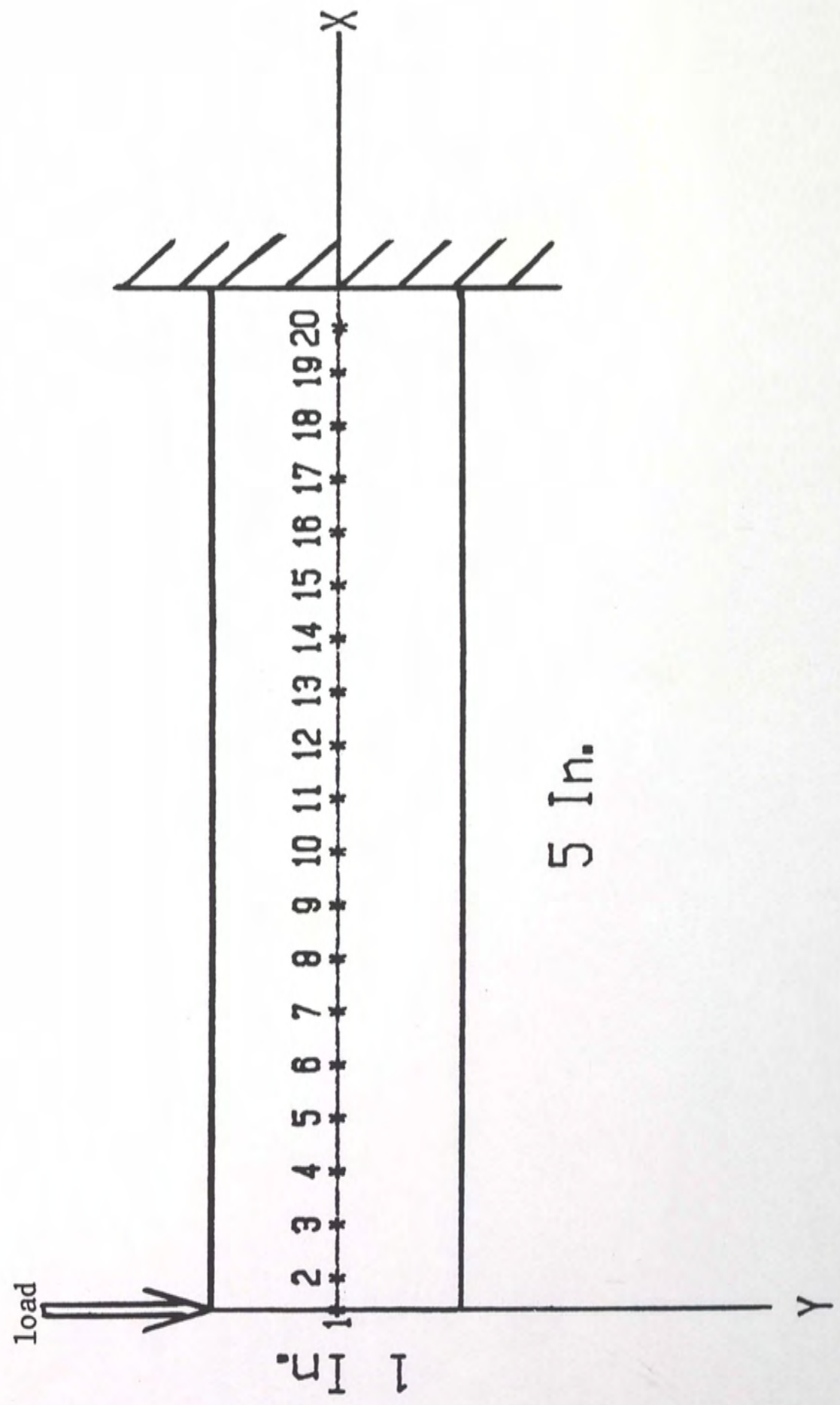



들

is

국

0

号

등

見

㟧

4

見

on

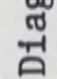

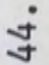

荡 

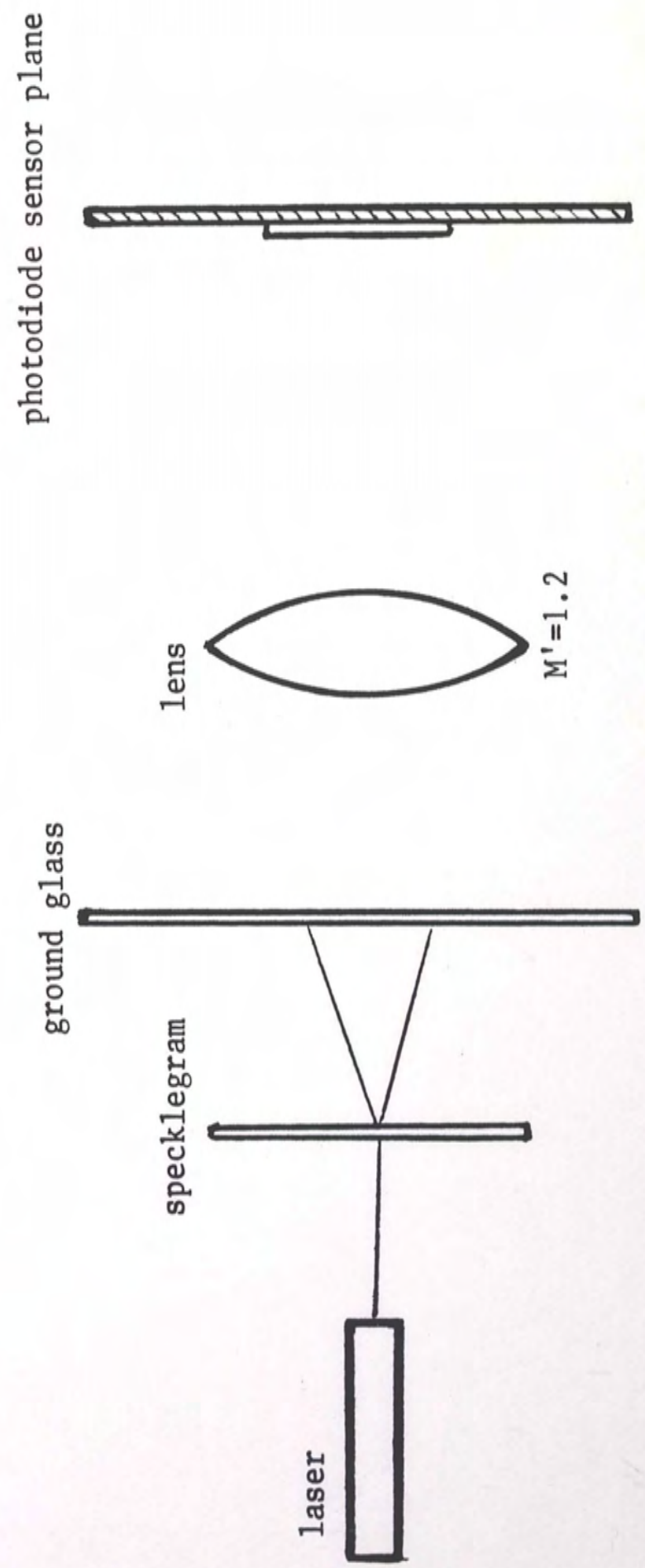

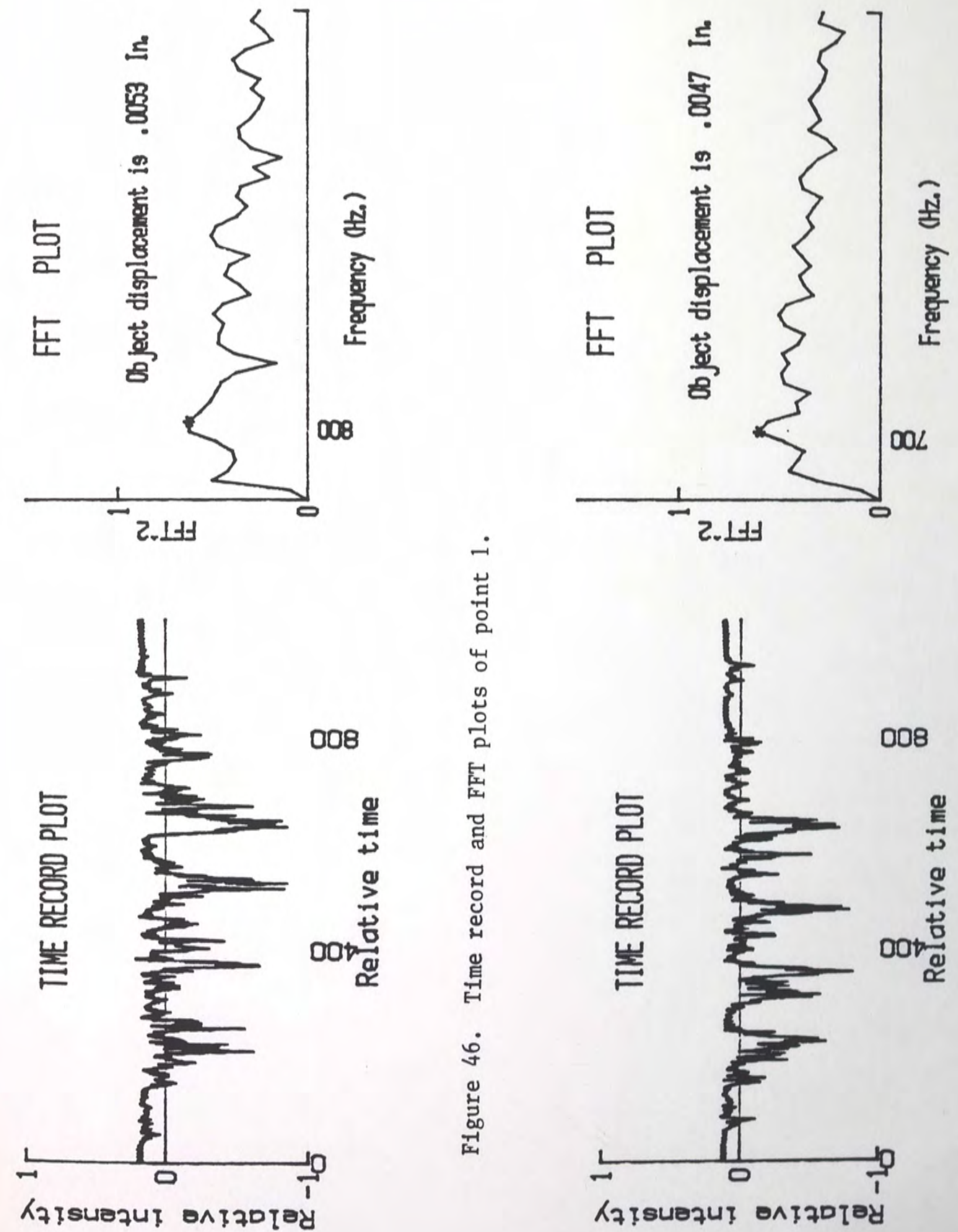

ㄱ. 

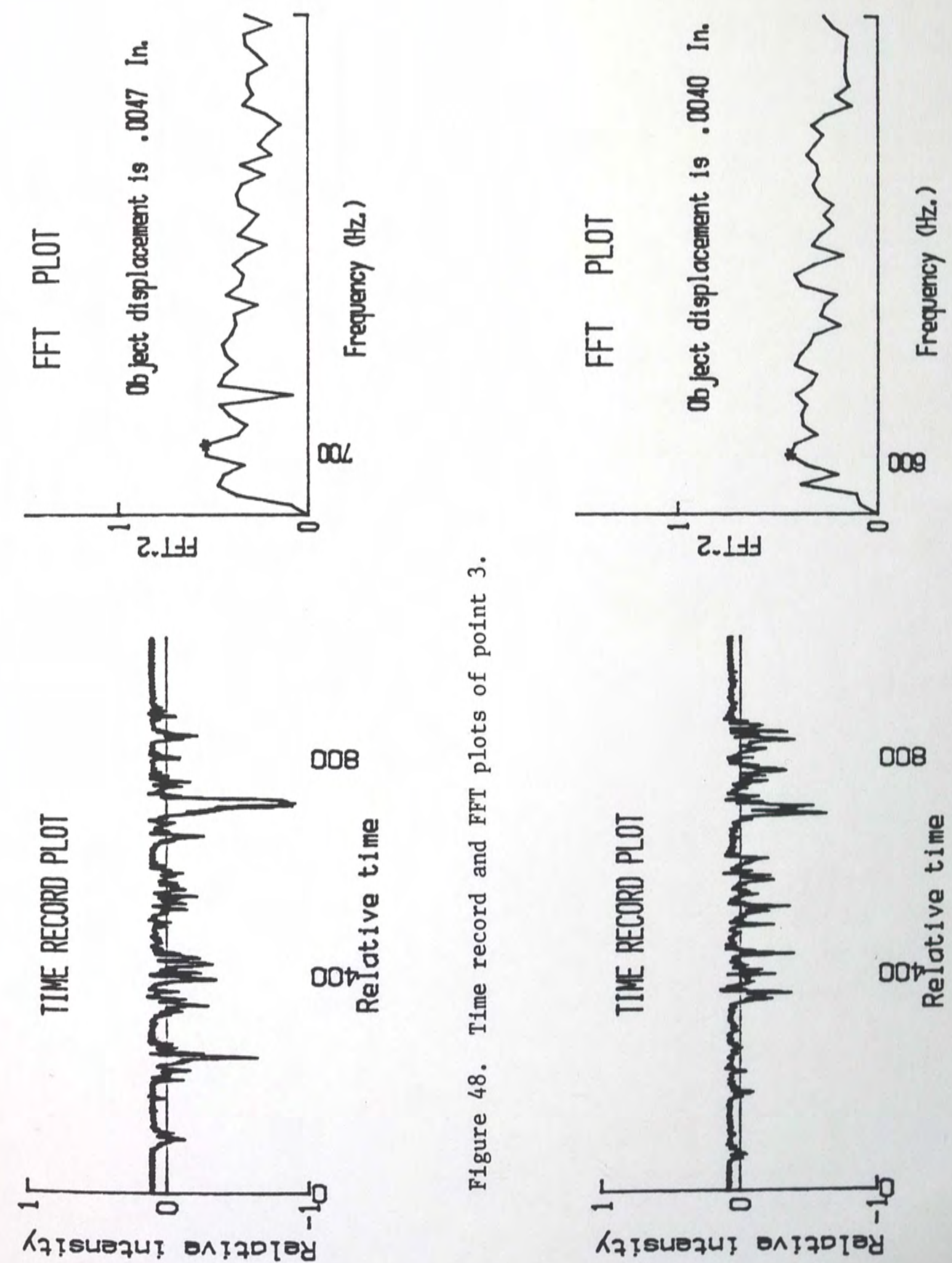

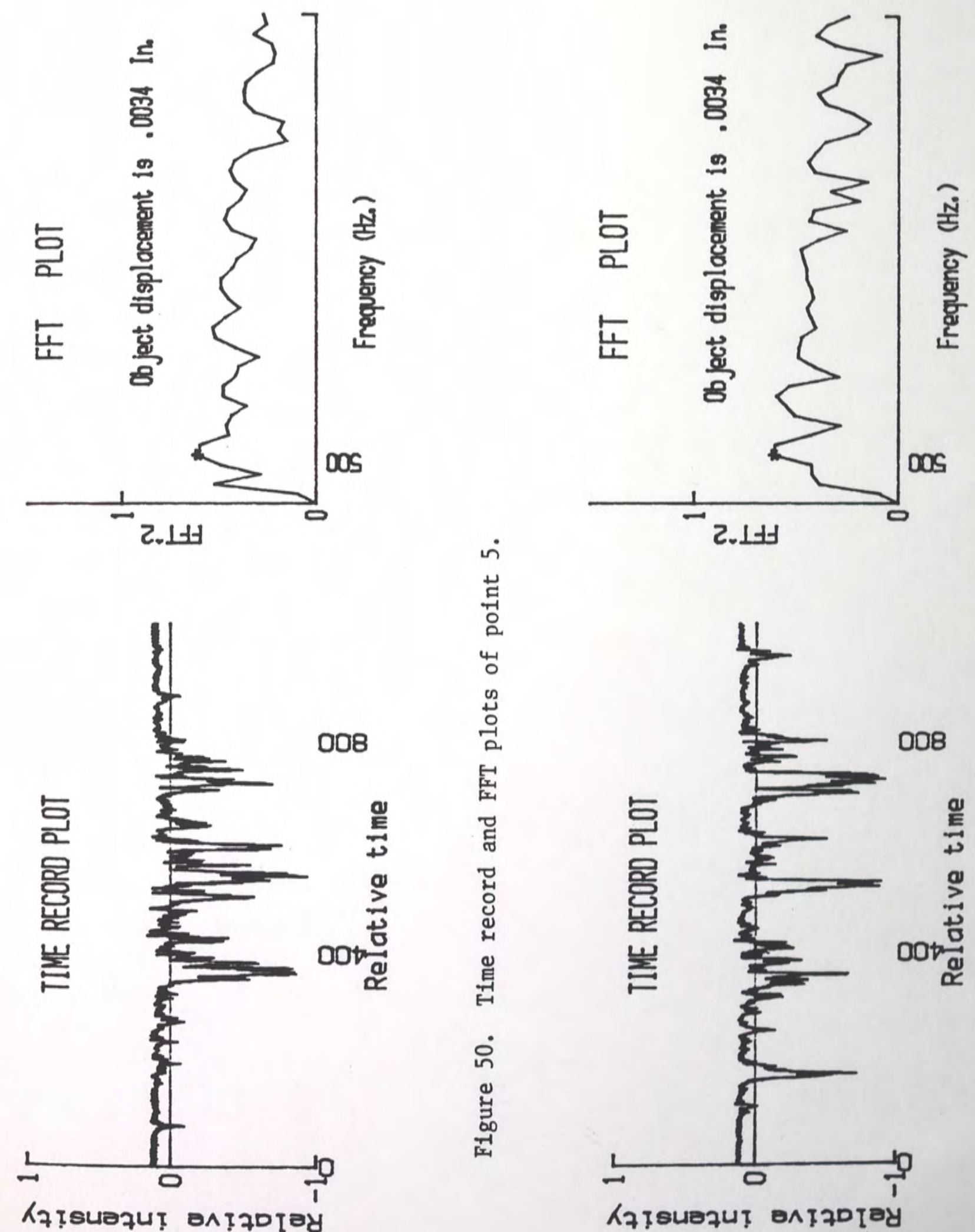

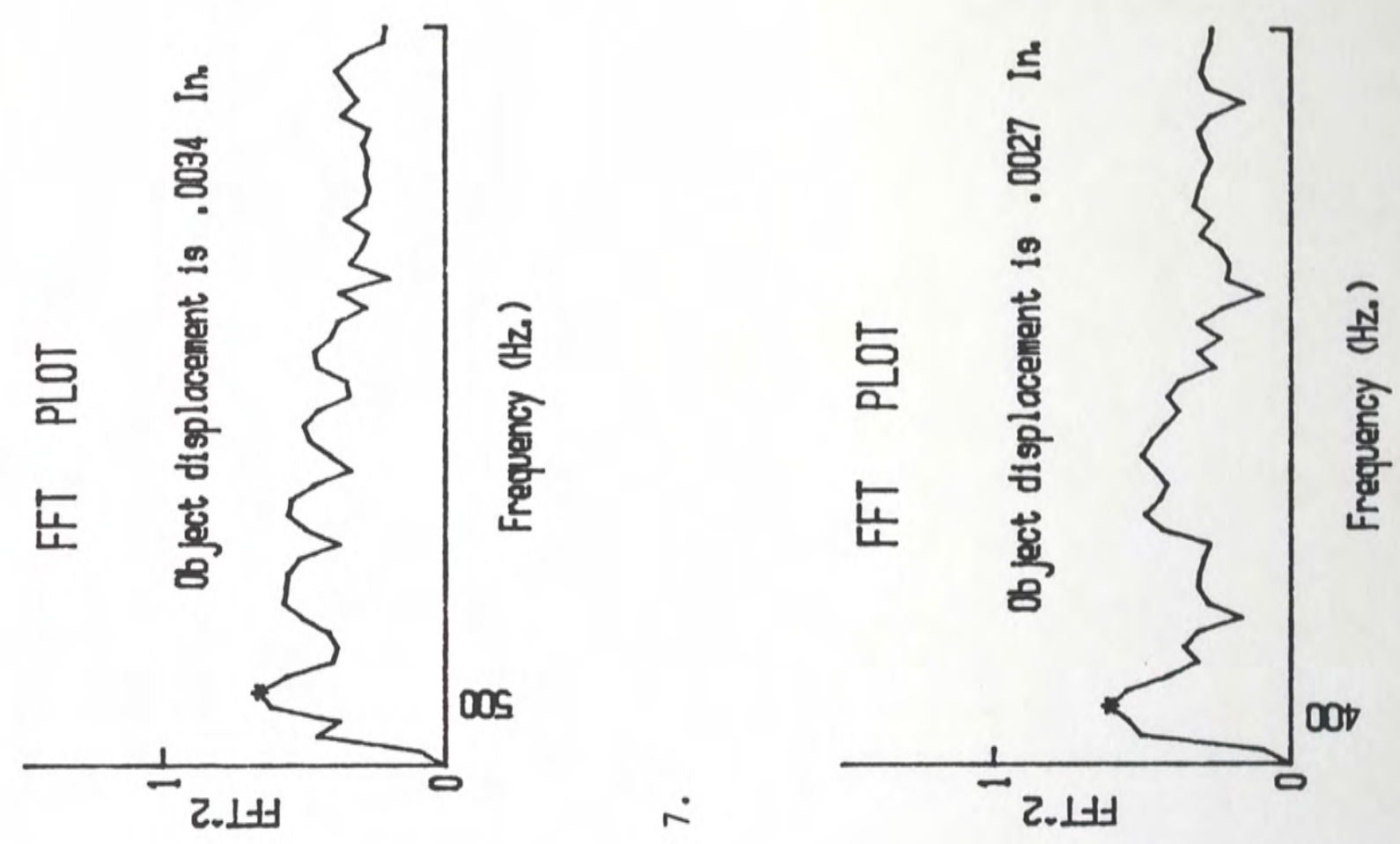

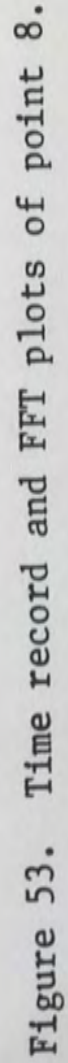

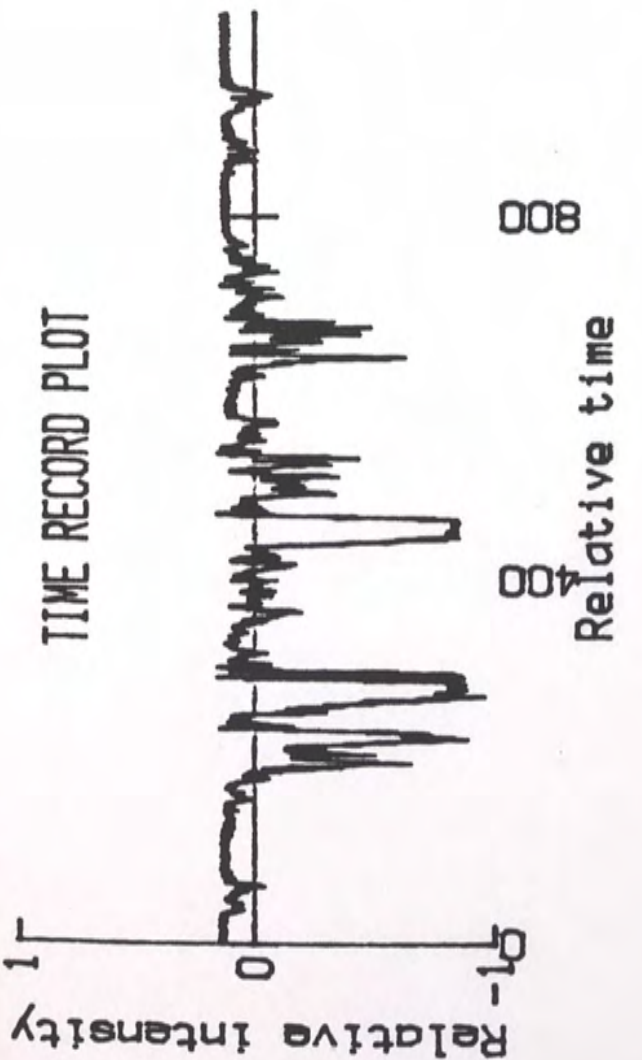

008

i

岂

㟧

雚

宦

믐

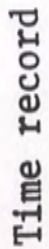

กำ

荡

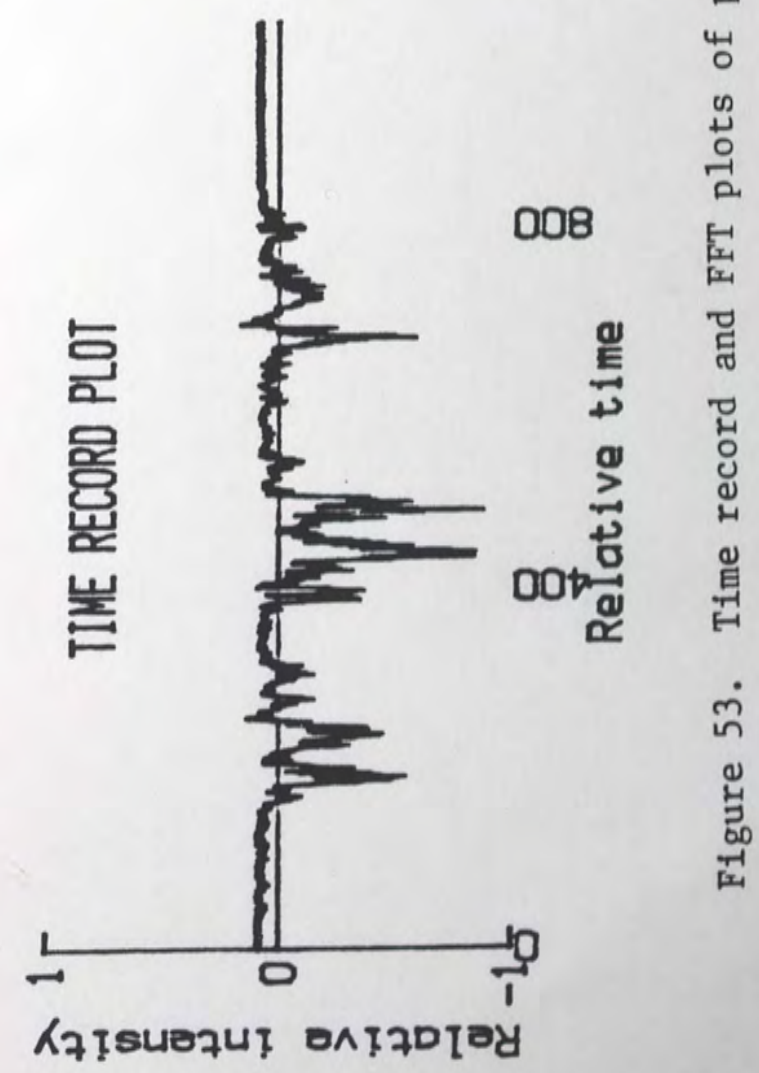




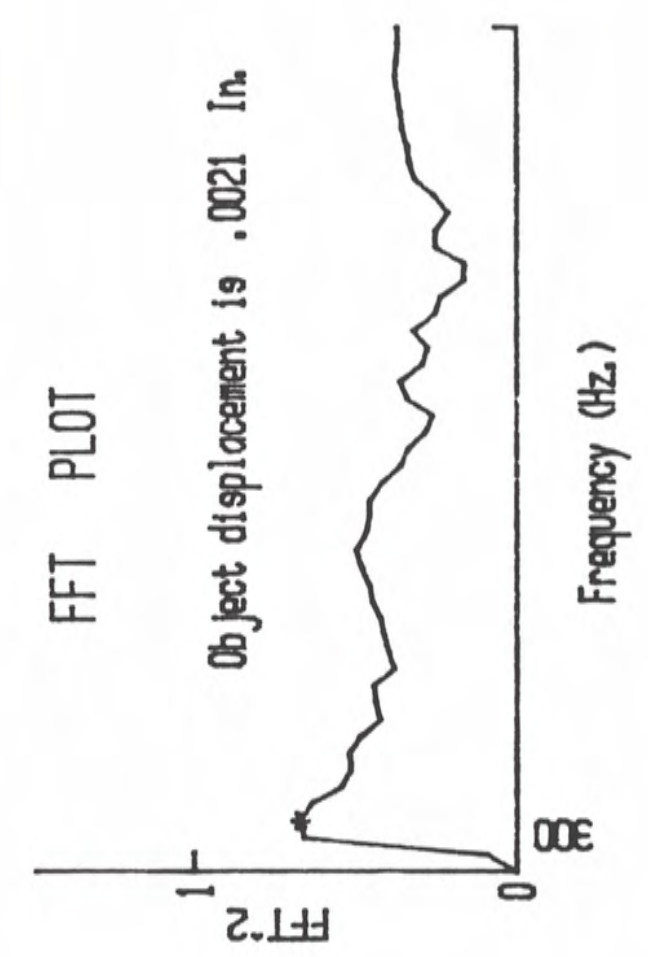

م.
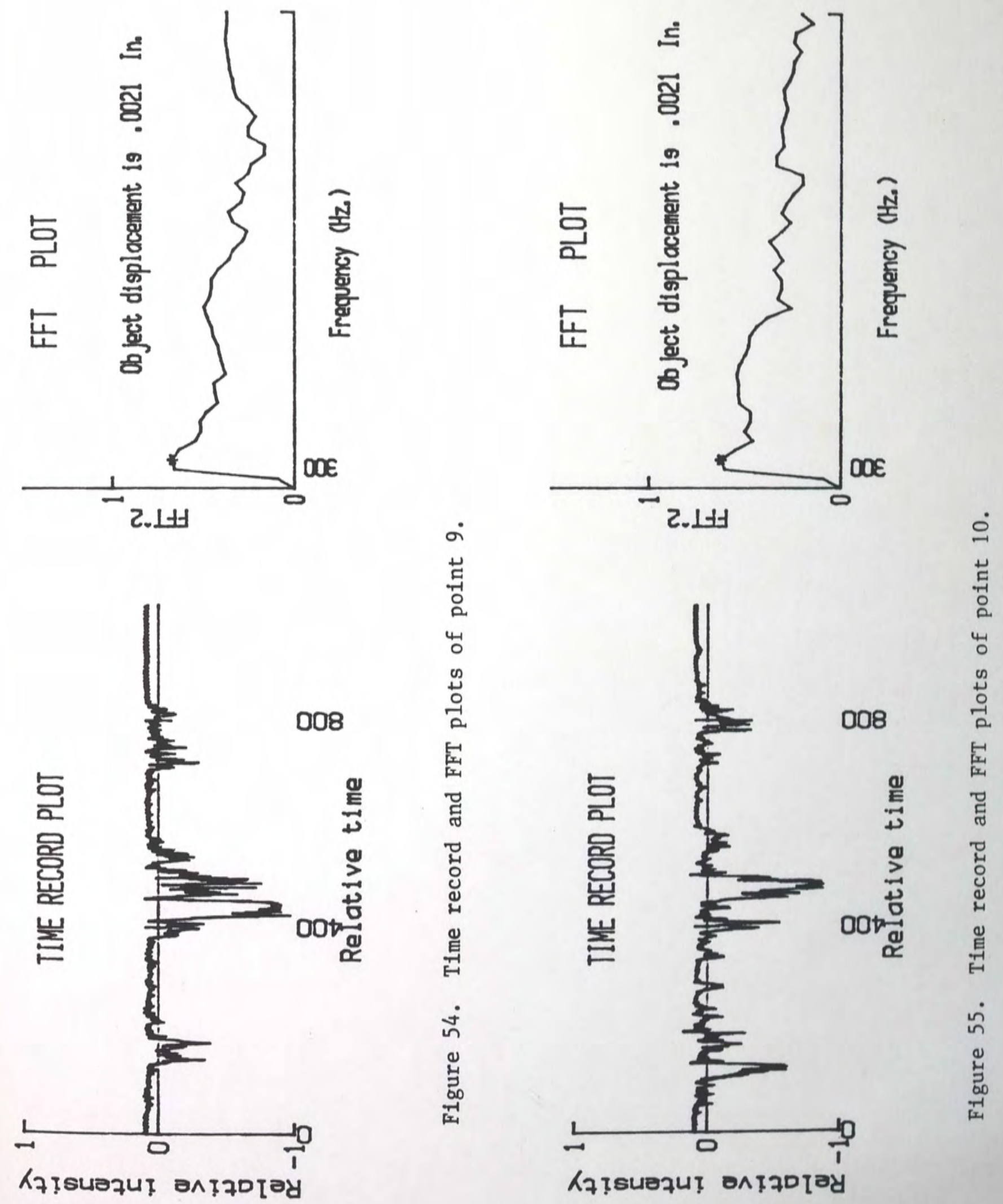

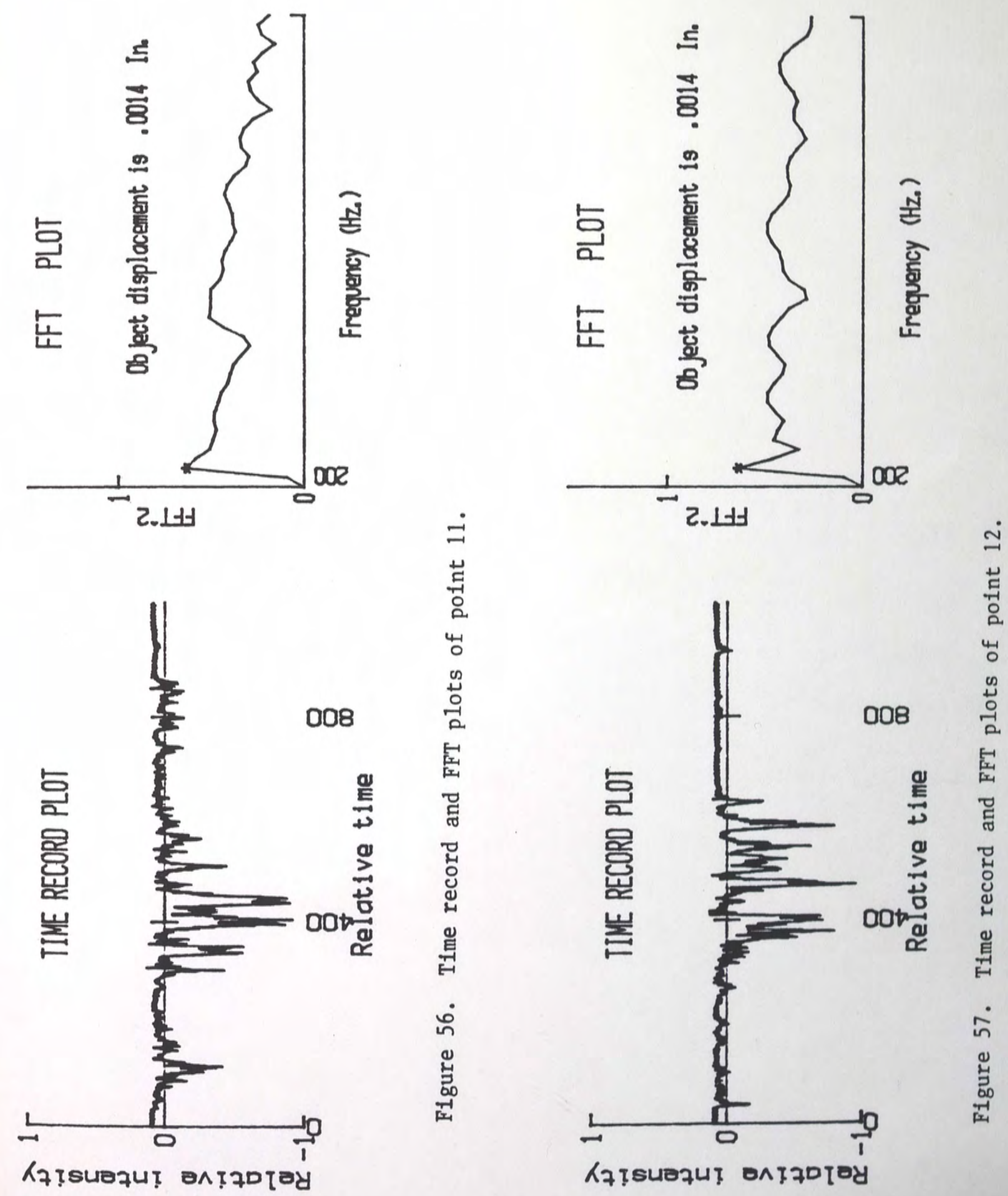

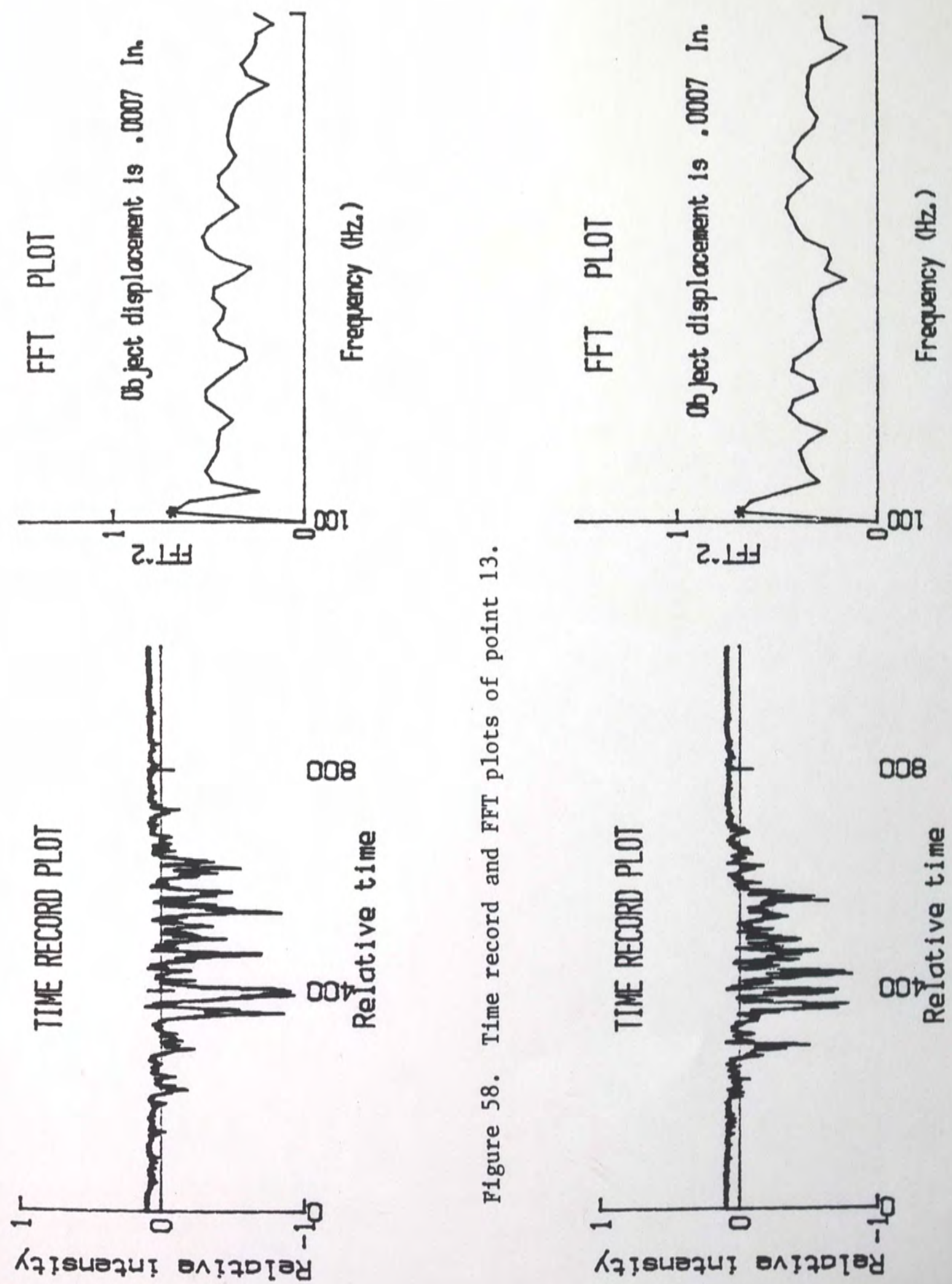

年

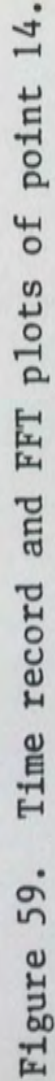



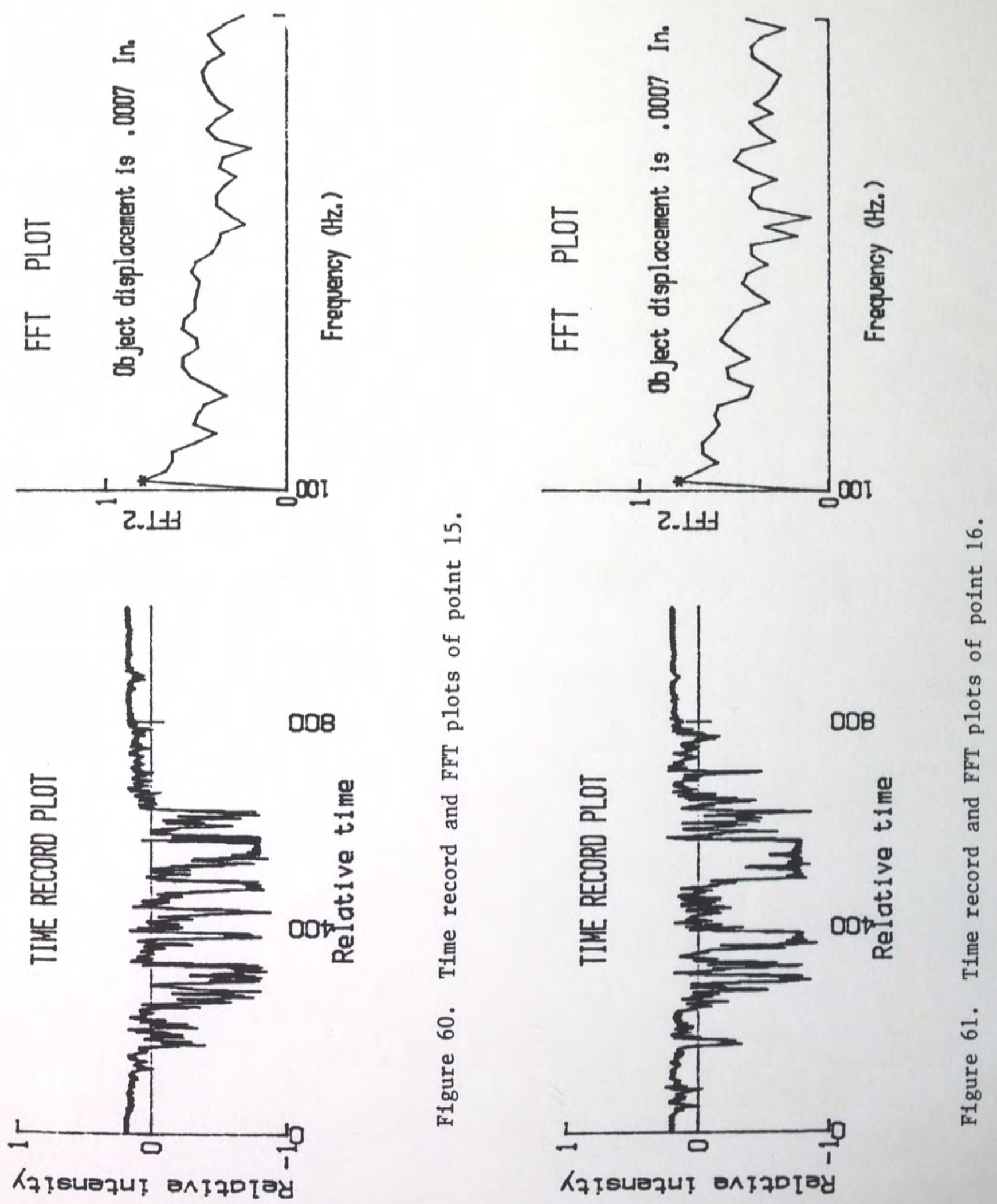

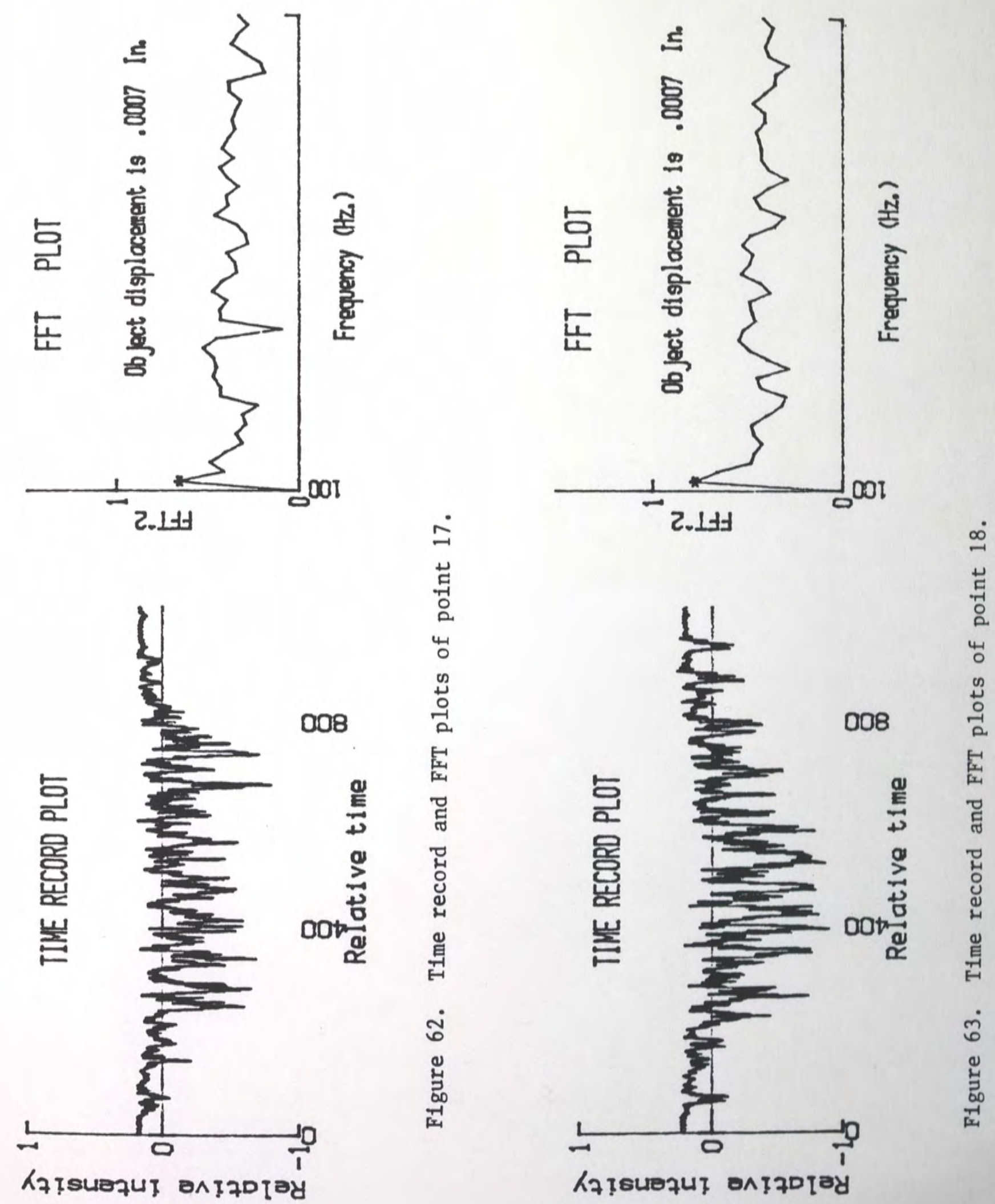

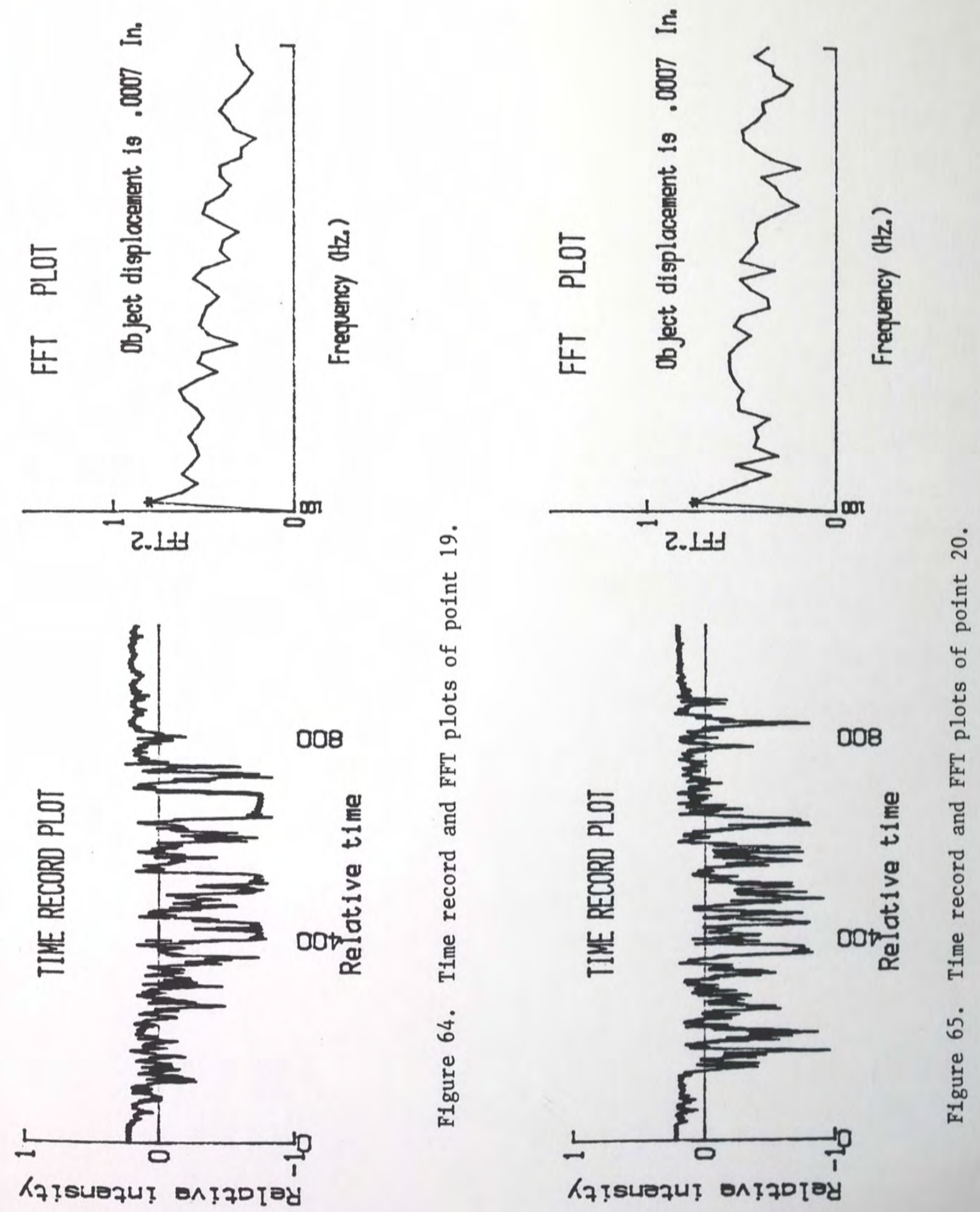
derived from the artificial fringes experiment. Knowing the fringe spacing, d' (on the sensor plane), laser wavelength, $\lambda$, distance, $z$, between the viewing plane (ground glass) and the specklegram, and the magnification factor, $M$, used in producing the specklegram, and beam deflection can be calculated using the following equation:

$$
v=\frac{\lambda z}{M d}=\frac{\lambda z}{M^{\prime} \frac{1}{M^{\prime}}}=\frac{M^{\prime}}{M} \frac{\lambda z}{d^{\prime}}
$$

Table 3 represents the computed experimental displacement values and the theoretical displacement values obtained from the beam theory as expressed by [44]:

$$
v=\frac{P x^{3}}{6 E I}-\frac{P x I^{2}}{2 E I}+\frac{P 1^{3}}{3 E I}
$$

where:

$$
\begin{aligned}
& \mathrm{v}=\text { vertical deflection } \\
& \mathrm{P}=\text { applied force } \\
& \mathrm{E}=\text { modulus of elasticity } \\
& \mathrm{I}=\text { moment of inertia } \\
& \mathrm{I}=\text { beam length }
\end{aligned}
$$

for a cantilever beam with an end load applied at the free end. The vertical deflection at the free end was 0.005 inch. Theoretical and experimental values of the vertical displacement of the centerline of the beam are plotted in Figure 66. As shown in the figure, experimental values compare favorably well with the theoretical 
TABLE 3

EXPERIMENTAL VERSUS THEORETICAL DISPLACEMENT VALUES AT DIFFERENT LOCATIONS ALONG CENTERLINE OF THE BEAM

\begin{tabular}{|c|c|c|}
\hline $\begin{array}{l}\text { BEAM LENGTH } \\
\text { (in) }\end{array}$ & $\begin{array}{c}\text { BEAM THEORY } \\
\text { THEORETICAL } \\
\text { (in) }\end{array}$ & $\begin{array}{l}\text { EXPERIMENTAL } \\
\text { (in) }\end{array}$ \\
\hline 0.00 & 0.0050 & 0.0053 \\
\hline 0.26 & 0.0046 & 0.0047 \\
\hline 0.52 & 0.0042 & 0.0047 \\
\hline 0.78 & 0.0038 & 0.0040 \\
\hline 1.04 & 0.0035 & 0.0034 \\
\hline 1.30 & 0.0031 & 0.0034 \\
\hline 1.56 & 0.0027 & 0.0034 \\
\hline 1.82 & 0.0024 & 0.0027 \\
\hline 2.08 & 0.0021 & 0.0021 \\
\hline 2.34 & 0.0018 & 0.0021 \\
\hline 2.60 & 0.0015 & 0.0014 \\
\hline 2.86 & 0.0012 & 0.0014 \\
\hline 3.12 & 0.0009 & 0.0007 \\
\hline 3.38 & 0.0007 & 0.0007 \\
\hline 3.64 & 0.0005 & 0.0007 \\
\hline 3.90 & 0.0003 & 0.0007 \\
\hline 4.16 & 0.0002 & 0.0007 \\
\hline 4.42 & 0.0001 & 0.0007 \\
\hline 4.68 & 0.0000 & 0.0007 \\
\hline 4.85 & 0.0000 & 0.0007 \\
\hline
\end{tabular}




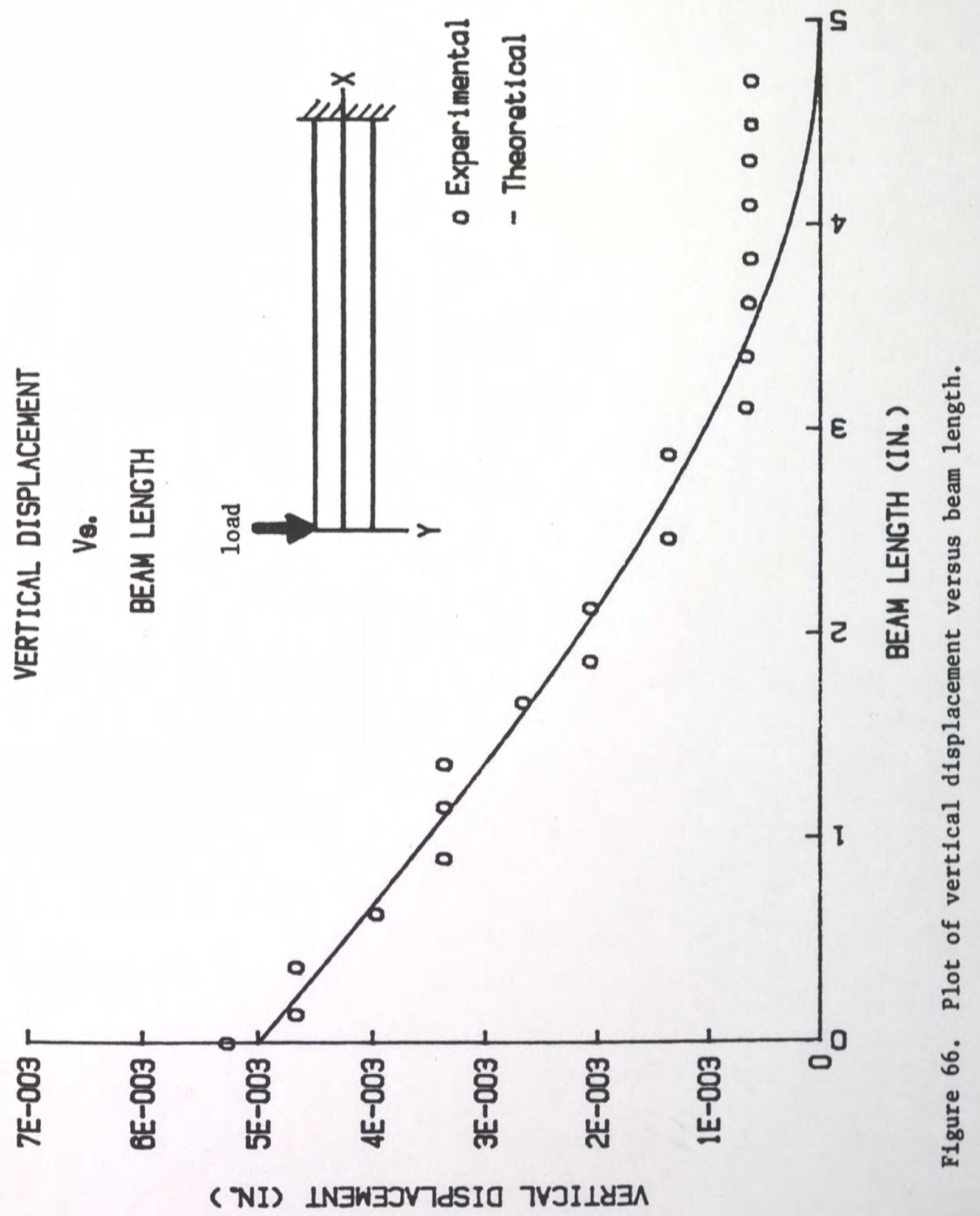


values, except near the fixed end. Displacements near the fixed end are small and, therefore, the resulted fringe spacing is large which, in turn, yields a small value for the fundamental frequency. Such a small frequency was unresolvable by the spectrum analyzer. Indeed, the spectrum analyzer (HP-3582A) was unable to record and display frequencies between 0 and $100 \mathrm{~Hz}$. 
CHAPTER VI

CONCLUSION AND RECOMMENDATIONS

An automated system for direct calculation of object displacement from double-exposure specklegram was developed. He-Ne laser and computer-controlled translation stages holding the specklegram represent the first part of the system, which is the optical Fourier processor. A CCD linear diode sensor is used to digitize the fringe pattern. Finally, a microcomputer and a spectrum analyzer are used to perform the analysis.

Fringe analysis using the Fourier transform method was shown to be effective for displacement measurement. To show the effectiveness of the technique, a series of related experiments were conducted. Among these experiments were the artificial fringe experiments which showed a linear relationship between the fringe spacing and the inverse of frequency. This relationship not only showed that the photodiode sensor was working properly, but also was used to approximate the fringe spacing when frequency was known.

To find the relationship between fringe number and frequency, five different cases $(3,4,5,6$ and 7 fringes) were tested and showed that the relationship between the number of fringes and the frequency was linear. 
Furthermore, average of square FFT records of eight scans on the fringe halo, for the purpose of decreasing speckle noise, showed that the FFT record was somewhat improved, but the location of the peak in the FFT plot did not change, due to the limited resolution of the spectrum analyzer.

Finally, cantilever beam deflection was measured. The procedure followed was to select a point on the beam neutral axis, obtain a time record from fringe digitizing, and then Fourier transform the time record. The resulting frequency is to be substituted into the previously obtained equation for fringe spacing to find the fringe spacing. From such information, beam deflection can easily be calculated. Results showed that the experimental values were very close to the theoretical values. The only non-uniformity noticed was due to the fact that the spectrum analyzer did not display or record the exact value of frequency between 0 and $100 \mathrm{~Hz}$. Therefore, beyond 3.7 inches from the free end, deflection was shown to be 0.0007 inch. Possible future extensions of this research may include measuring deflection at any location on the beam. Such measurements may include calculation of the angle between $x$ and $y$ components of the displacement. Displacement information may be used in conjunction with numerical techniques such as the boundary element method or the finite element method to yield the stress value at any desired location. 
APPENDICES 
APPENDIX A

OPERATION OF CC-1 CONTROLLABLE TRANSLATION STAGES

The Klinger Scientific's Model CC-1 programmable stepper motor controller is designed to control stepping motors in response to commands, either from computer or from front panel pushbutton controls. When used in conjunction with Klinger's stepping motor driven positioning stages, they allow for precision single- or dualaxis micropositioning.

The CC-1 controller, when operated from a computer or other data source capable of supplying ASCII code or binary code commands, can perform the following:

1. Establish a home reference position and make all movements relative to this home position.

2. Perform movements relative to the present position in positive or negative direction.

3. Execute movements over a wide speed range (400-2000 Hz) with varying acceleration range (0-0.1 sec).

4. Place a delay of any length between successive movements.

5. Establishes number of steps to be taken in a relative motion (1-65535 microns).

The CC-1 has three programming modes:

1. Command mode

2. Program entry mode

3. Program execution mode 
In the command mode, commands are executed as they are received. The CC-1 powers-up in command mode. In program entry mode, a sequence of commands is entered and stored in a program buffer for later execution. There are 48 bytes of memory available for program storage. Table 1 shows the required commands and the number of bytes required for each command. It is impressive to know the length and, correspondingly, the location of each instruction or command to do looping and branching. In program execution mode, there are two commands that enable the entry of this mode. One command, "D $\downarrow$," starts program execution from location zero of the program storage buffer, and the other, "O $\downarrow, "$ starts program execution from the location specified in the command.

The CC-1 allows the set-up of the step rate and acceleration rate in very fine increments (10 microsecond changes). This is easily done through the rate, slope and factor ( $R, S$ and $F$ ) commands. By proper choice of the $R, S$ and $F$ commands, it is possible to control the starting rate, the number of steps from the starting rate to the slew rate and the final slew rate.

The CC-1 rate equation is:

$$
\text { step/second }=\frac{10^{6}}{(256-r) \cdot 80+z \cdot 10+75 \pm 7.5}
$$

where:

$$
\begin{aligned}
& r=\text { rate parameter as set by the } R \text { command } \\
& \mathrm{s}=\text { slope parameter as set by the } \mathrm{S} \text { command }
\end{aligned}
$$




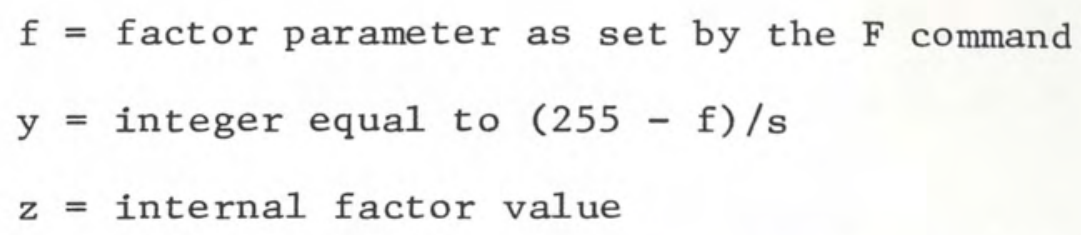

The factor y represents the number of steps required to ramp up from the starting rate to the slew rate, and the number of steps to ramp down again better stepping; and the internal factor, $z$, changes in value for each step while ramping. When accelerating:

$$
\mathrm{Z}=255-\mathrm{n} \cdot \mathrm{s}
$$

where:

$\mathrm{n}=$ step number from the start motion beginning with $\mathrm{n}=1$ When $z=f$ (slew speed), the maximum step rate for any particular value of $\mathrm{r}$ and $\mathrm{f}$ has been reached. When decelerating:

$$
\mathrm{z}=\mathrm{f}+\mathrm{n} \cdot \mathrm{s}
$$

where:

$\mathrm{n}=$ step number from the start of deceleration

From the above discussion, the starting step rate equation can be deduced plus the final ( $\mathrm{slew}$ ) step rate equation.

Start step rate equation:

$$
\text { step/second }=\frac{10^{6}}{(256-r) \cdot 80+(255-s) \cdot 10+75 \pm 7.5}
$$

and slew rate equation: 


$$
\text { step } / \text { second }=\frac{10^{6}}{(256-r) \cdot 80+f \cdot 10+75 \pm 7.5}
$$

For example, for the following commands, "R 253," "S 1," "F 20 ," "G,", the slew rate is:

$$
\begin{aligned}
\text { step/second } & =10^{6} /(256-\mathrm{r}) \cdot 80+10 \cdot \mathrm{f}+75 \\
& =10^{6} /(256-253) \cdot 80+10(20)+75 \\
& =1914
\end{aligned}
$$

The CC-1 is used as a listener on the IEEE-48 bus line. To establish communication, the CC-1 must be properly configured through its dip switches. The dip switches are located on the back panel of the CC-1 controller (for exact location refer to the CC-1 operation manual). They should be set as follows:

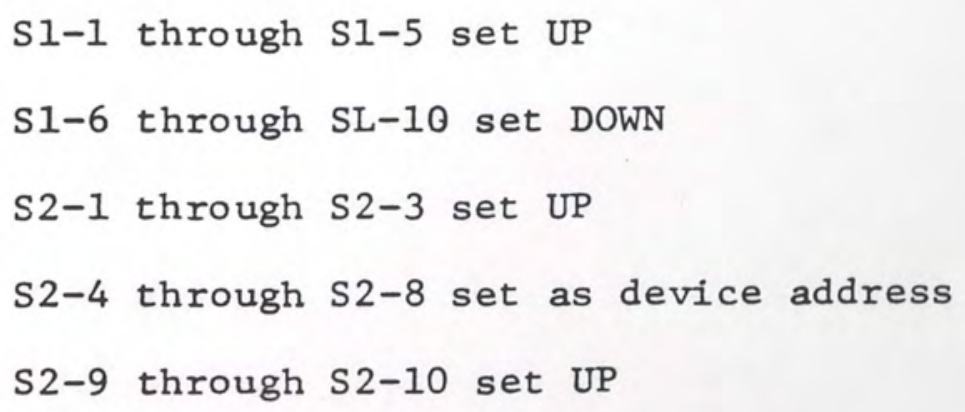


APPENDIX B

INSTRUCTIONS ON CCD-133 LINE SCANNER

Basically, a line scan image sensor is composed of a row of image-sensing elements (photosites), two analog transport registers and an output amplifier.

Light energy falls on the photosites and generates charge packets proportional to the light intensity. These charge packets are then transferred in parallel to two analog transport registers, which are checked by 2-phase clocks. The packets are next delivered to an on-chip output amplifier where they are converted to proportional voltage level. A series of pulses, amplitude modulated with the optical information, appear at the output.

Fairchild's charged-couple-device, or CCD, contains $1024 \times 1$ light sensitive elements of $13 \times 13$ microns. Its maximum auto rate is $20 \mathrm{MHz}$ with 2500:1 dynamic range, and 3.0 volts per microjoules per centimeter squared, respectively. The 1024 sensing elements of CCD-133 provide a 120-1ine per inch resolution across an $8-1 / 2$ inch page.

To understand the operation of CCD, refer to figures 67 and 68 . Figure 67 is the block diagram and Figure 68 is the timing diagram CCD-133. 


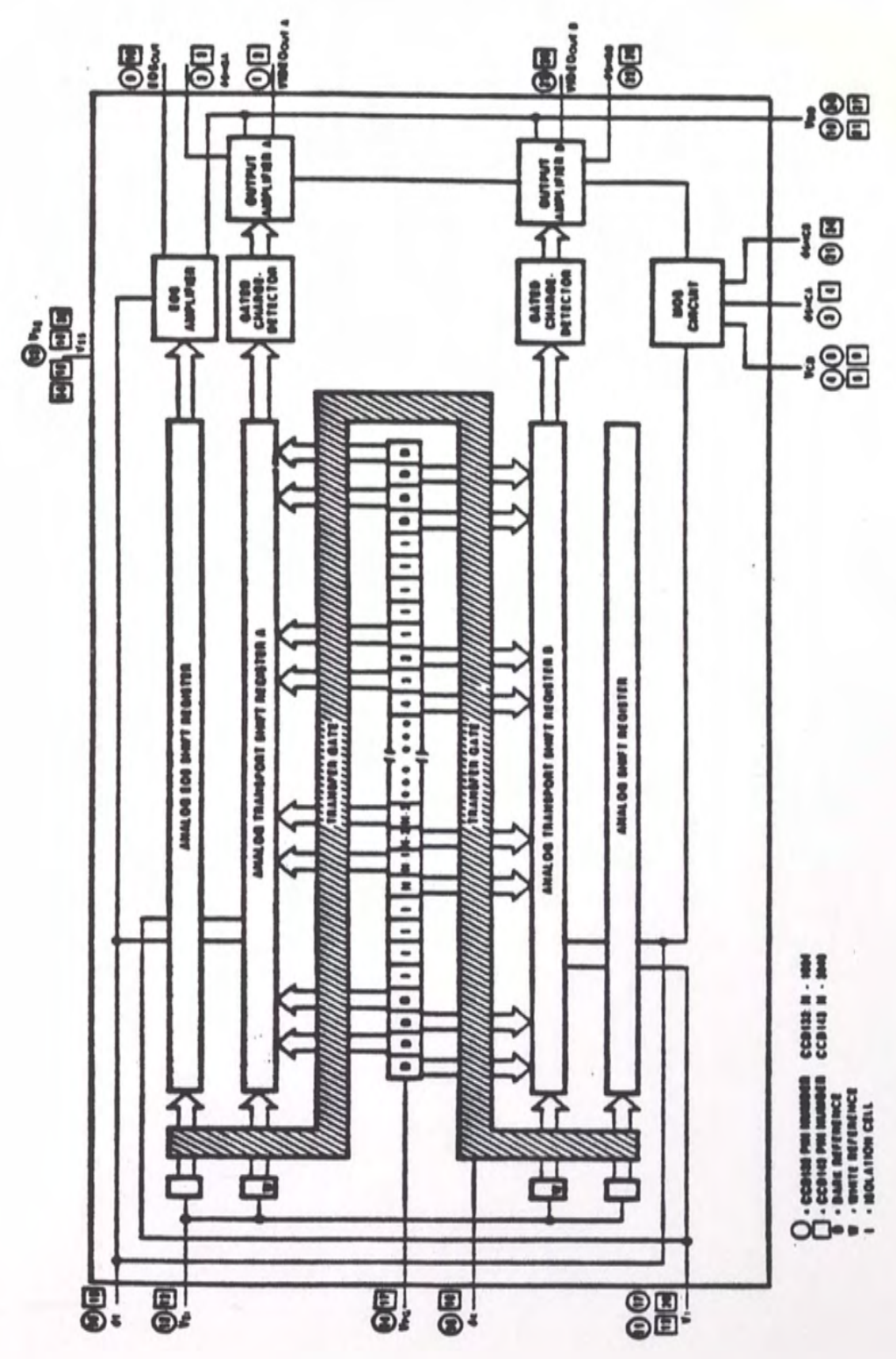

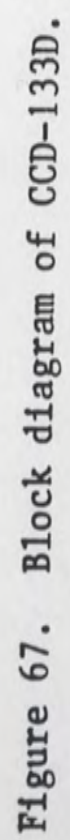




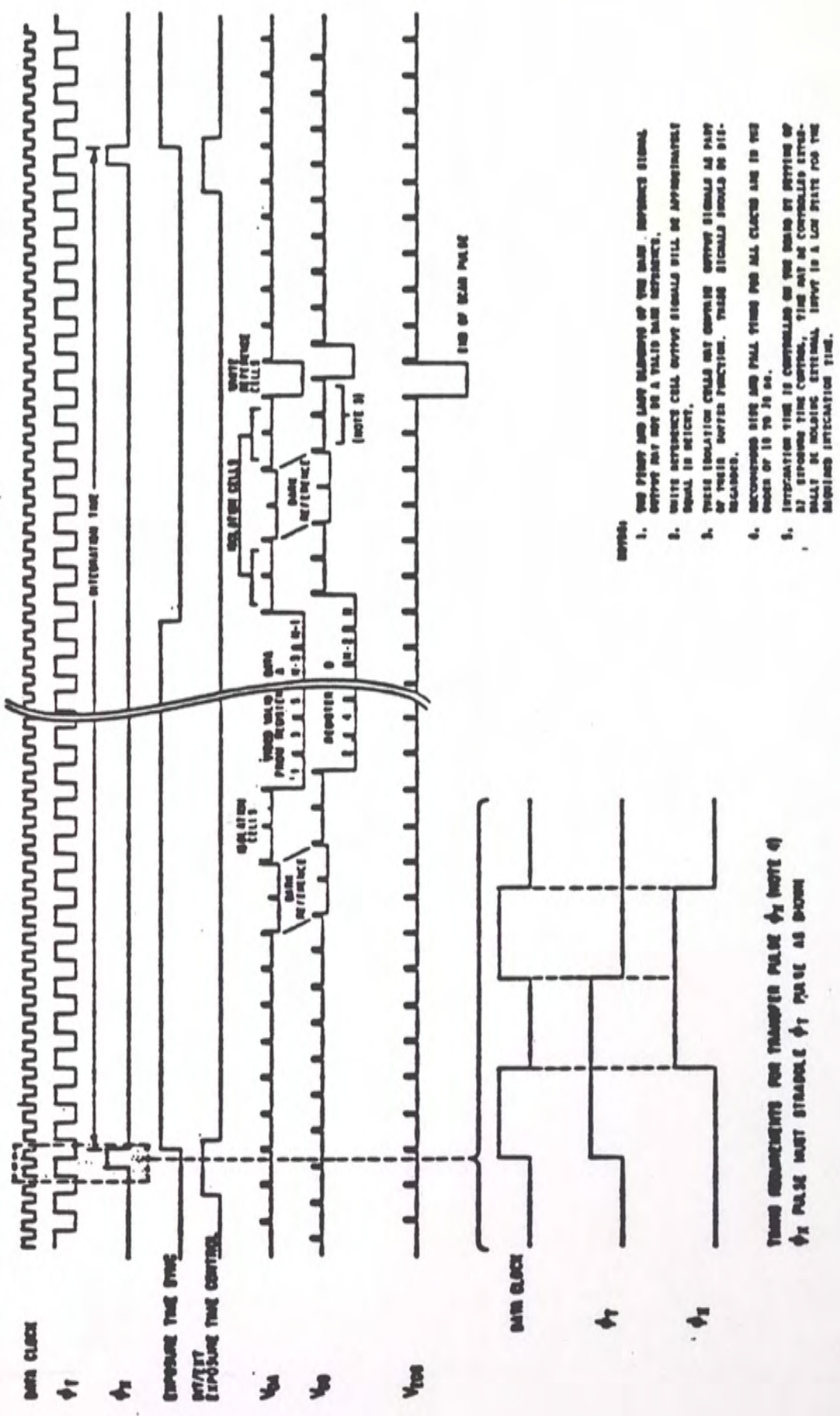

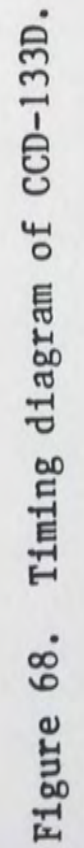


Image sensor elements or pixels are separated by diffused channel stops, and covered by a silicon dioxide surface passivation layer. Image photons pass through the transparent silicon dioxide layer and are absorbed in the single crystal silicon creating holeelement pairs. The charged-packets accumulated in the image sensor elements are transferred out via the transfer gate to the transport register whenever the transfer gate register goes high. Alternate charge-packets are transferred to the analog transport shift registers. Two registers are on each side of the line of image sensor elements and separated from it by the transfer gate. They are used to move the image generated charge-packets delivered by the transfer gate serially to the two charge-detector/amplifiers. There, the amplifiers allow the output to be delivered as a sample-and-hold wave form.

Figure 68 shows the clocks used in this board. $\phi_{x}$ is the transport clock, which is applied to the gates of the CCD transport shift registers to move the charge-packets received from the image sensor elements to the gated charge-detector amplifier, and $\phi_{\tau}$ is the transfer clock, which is the voltage waveform applied to the transfer gate to move the accumulated charge from the image sensor elements to the CCD transport shift registers. The two clocks, $V_{o a}$ and $V_{o b}$, are used for data transfer activation. They provide adequate support for detecting intensity as low as 300 microwatts. This amount is inadequate compared to 10 microwatts or lower needed in this 
experiment. To fix this problem, two function generators and one pulse generator were used, provided that pin 17 of the CCD board was grounded, and pins 3 and 5 connected to the function generators. First function generator was used to derive the pulse generated to account for exposure rate, and the second function generator was used for data rate control. In addition, an external trigger on the HP-3582A spectrum analyzer was needed to expand the time record signals derived from the sensor. Adjustment done on the two function generators and pulse generator was possible by reading data from the charts presented in Figure 69. These adjustments are shown in Figure 70. Figure 71 shows the schematic diagram of set-up. 


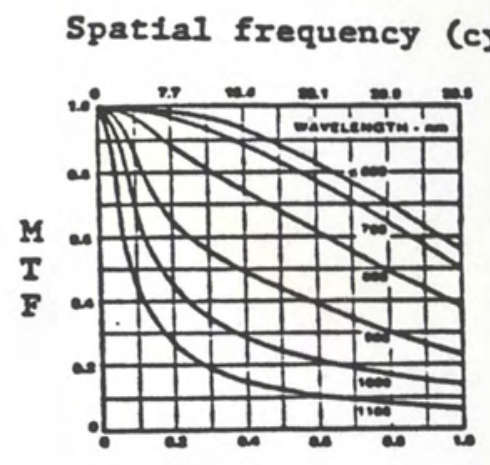

\section{Normalized spatial frequency (a)}
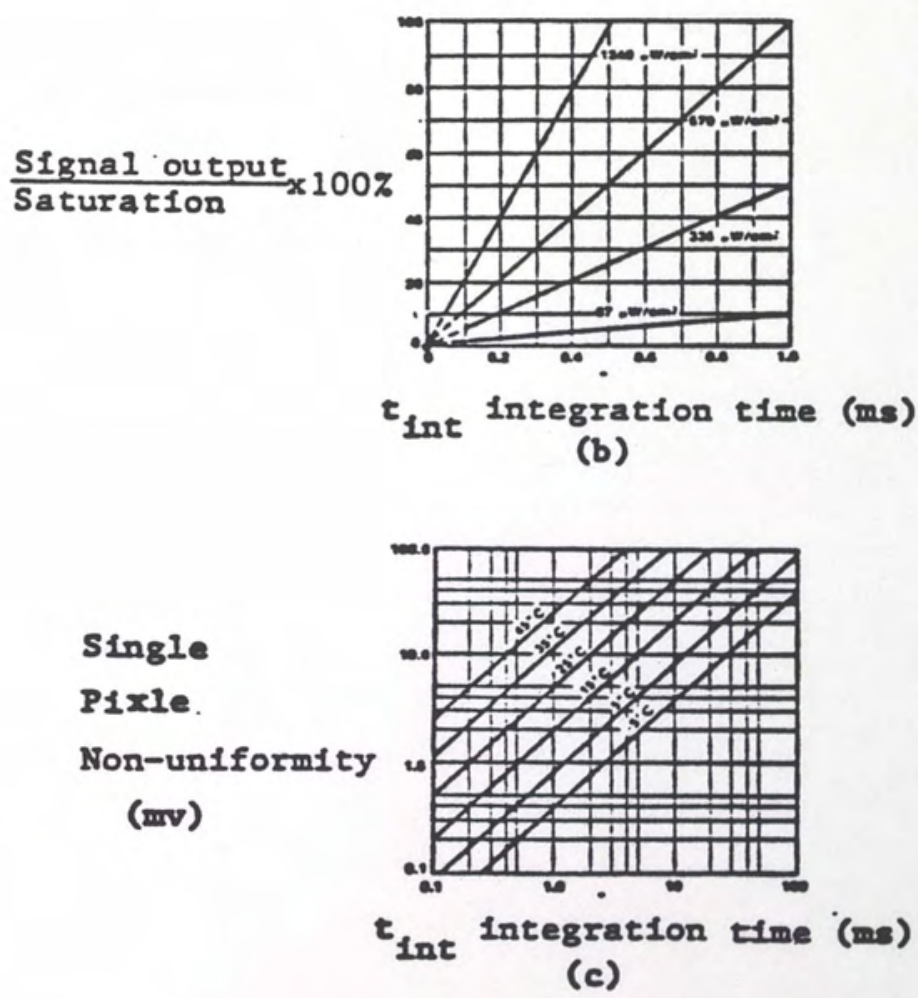

Figure 69. CCD-133D characteristic charts. 


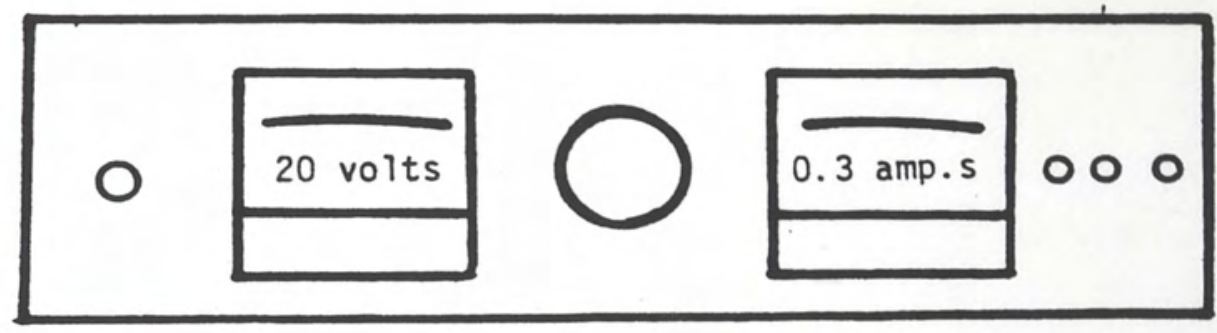

power supply

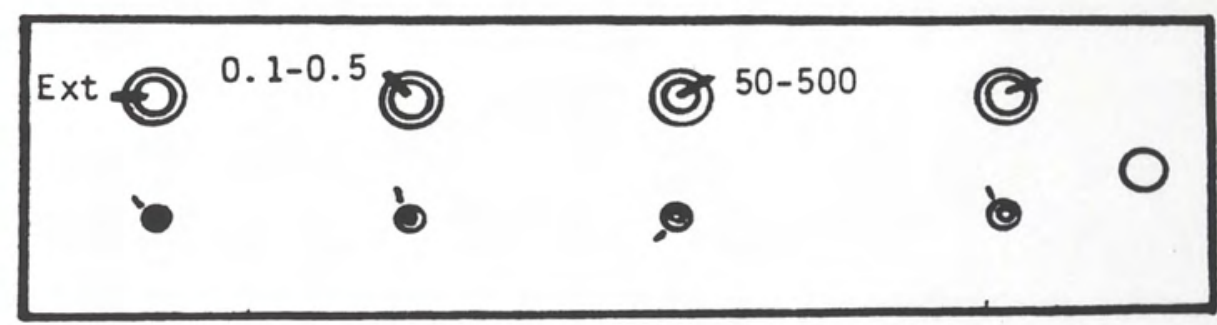

pulse generator
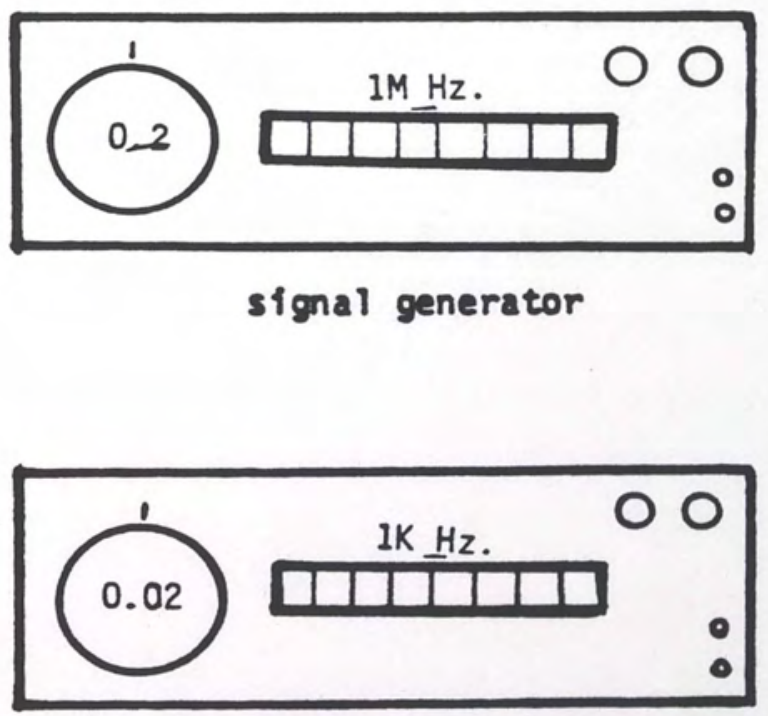

signal generator

F1gure 70. Equipment and their respective adjustments. 


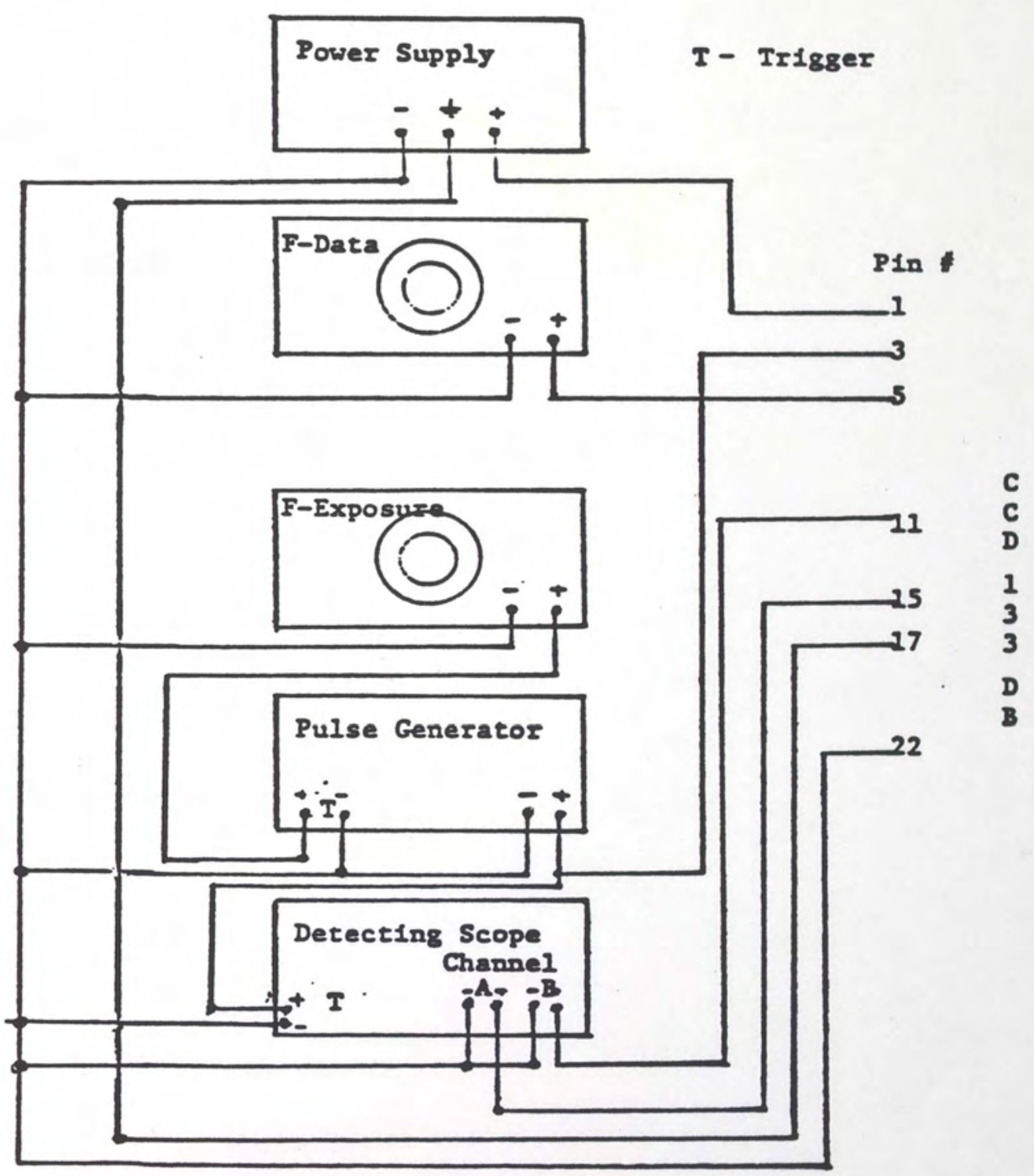

Figure 71. Schematic diagram of set up. 
APPENDIX C

DATA MANIPULATION PROGRAMS

Programs 1isted below were used to communicate with the HP-3582A and K1inger Scientific's CC-1 controllers. In their "LISTEN" mode, they are capable of receiving information from a microprocessor $(\mathrm{HP}-85)$, and in their "TALK" mode, they are capable of sending information. Both the spectrum analyzer and the translation stage controllers receive and send data in 16-bit, or two-byte words with least significant byte first. Hewlett-Packard microprocessor Model 85 is programmed in the BASIC language and is capable of advance programming through its matrix and memory expansion modules.

These programs are:

1. "STORDAT": This program has a routine to activate both the spectrum analyzer and the translation-stage controllers. In this routine, the operator is capable of scanning along the cantilever beam length every 5 millimeters. This interval can be changed by editing line 350 of this program, and replacing "N 5000 " by any integer $(0-65,535)$. Data file name has to be defined at the beginning of the program and it has to be any four-letter word.

2. "AUTOAVG": This routine can be used when the photodiode sensor is mounted on a translation stage. When this program is activated, it sends commands to the translation stage controller to move the sensor a specified distance. 
When the motion is completed, it stores the data and moves to a new position. When two consecutive scans are completed, it adds and averages the data in a datafile called "DATA100." Upon completion, the translation stage is moved to a new position collecting another two sets of data which are averaged and stored in a new datafile called "DATA200." These two files are recalled and then averaged, and their averages are stored in a datafile arbitrarily chosen by the operator at the beginning of the program.

3. "AutoAvg4": Similar to "AUTOAVG," this routine takes the average of four scans to the right of the bright spot in the fringe halo and stores it. Data collected from this program and "AUTOAVG" averaged further utilizing "NEWDATA" program; therefore, resulting average of eight scans.

4. "SCANAVG": Program "SCANAVG" is a modification of "AUTOAVG." In this program, automatic control routine has been removed. Physically, the automatic translation stage is replaced by a manual translation stage. Every time a scan is completed, a message is prompted on the computer screen notifying the operator to move the sensor to a new position.

5. "TRANS1" [45]: Once data is stored on a disk, it can be recalled and viewed. This program was prepared to analyze and view the data. It consists of eight options briefly discussed below.

a. K1 (DATA ENTRY): This routine allows data entry from the spectrum analyzer, from the datafile or from the keyboard. 
b. K2 (REMOT CONTROL): This option enables the operator to remotely operate the analyzer for data analysis or manipulation.

c. K3 (TIME RECORD PLOT): This option plots the time record directly from the analyzer CRT, or from the datafile.

d. K4 (FAST FOURIER TRANSFORM PLOT): This routine Fourier transforms the time record data and then plots it.

e. K5 (SAVE): Time record data, in binary form, is stored on a disk when this routine is activated.

6. "PlotTING": This program plots the Fourier transform and the time record data. Upon activation, the time record data is zero meaned first and then Fourier transformed. The Fourier transform plot shows the maximum peak occurrence and the object displacement.

7. "DATPLOT": Some times, it is necessary to compare two data points against each other. This program is written especially to plot the Fourier transform and the time record of two different data on the same sheet. Both data are plotted and are zero meaned.

8. "PLOT": This routine was prepared to plot the fringe spacing versus the inverse of frequency. This program plots the data collected from the artificial fringe experiment. It also shows the line representing the best fitted graph along with its equation. 


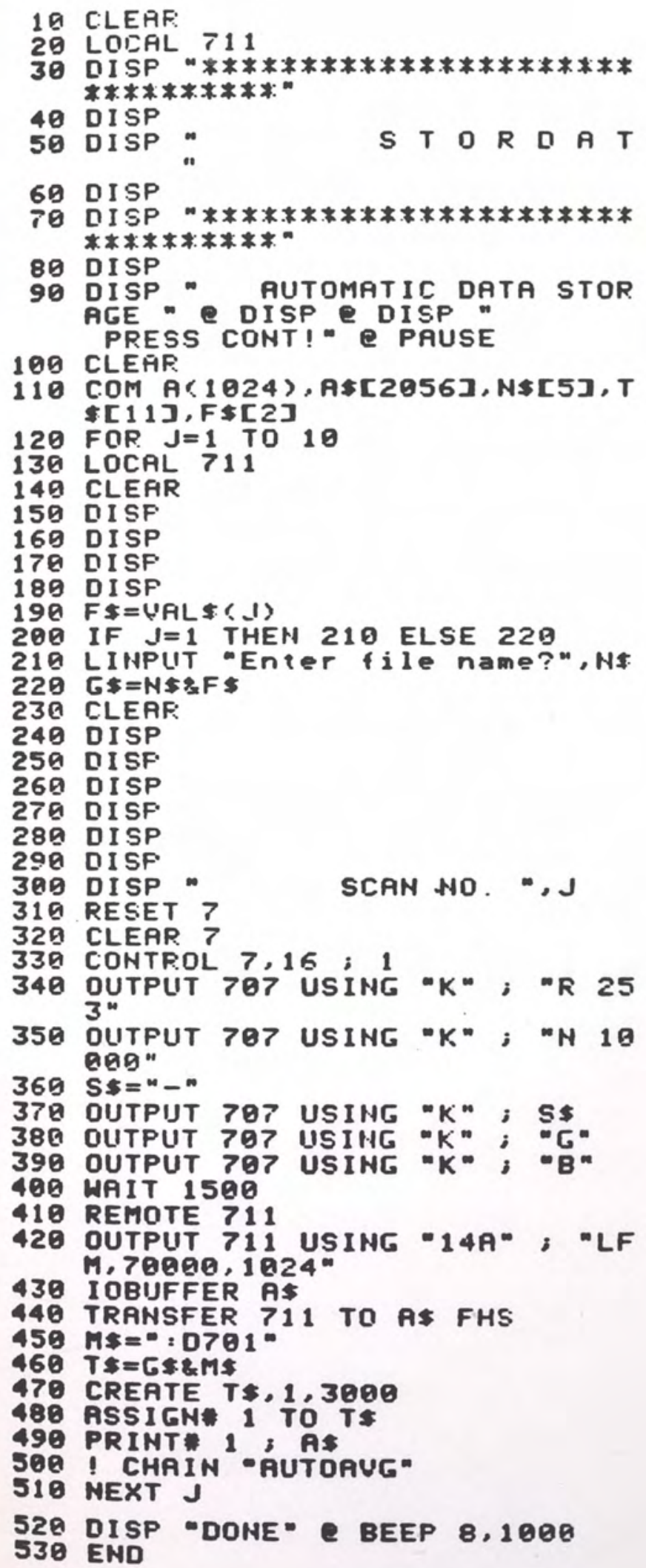




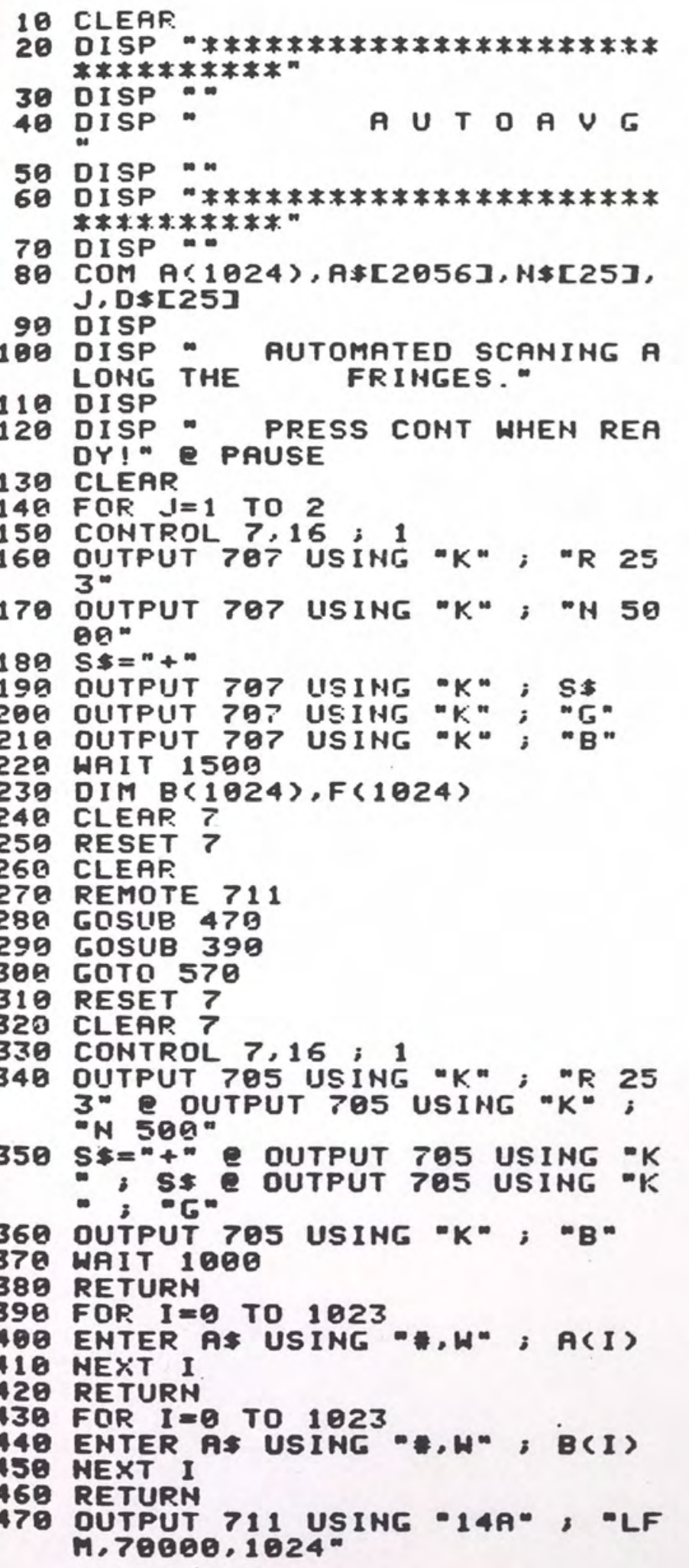




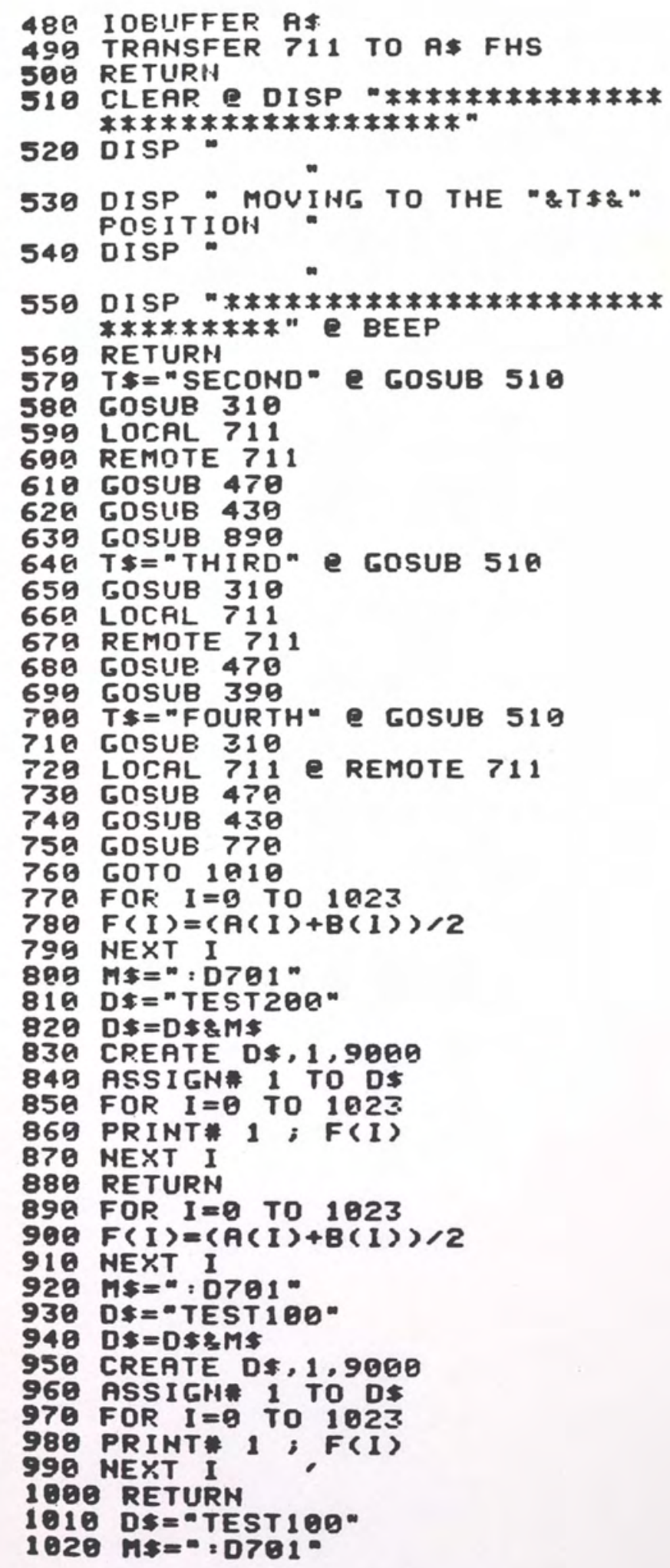




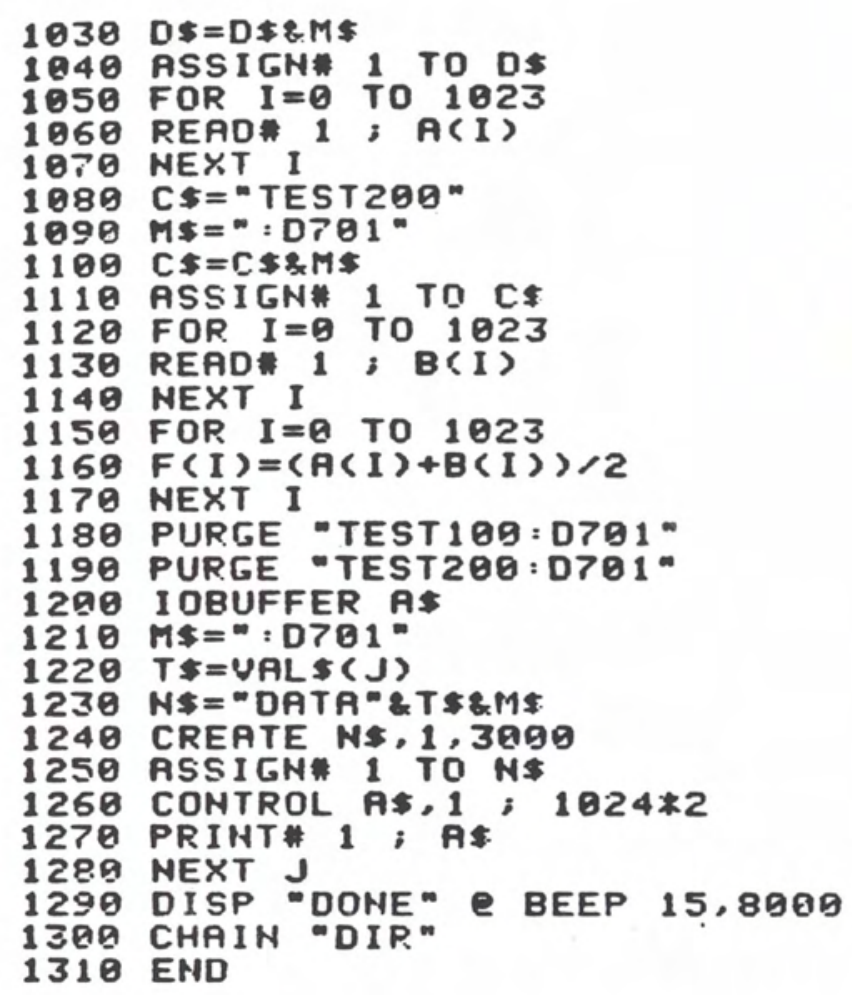


5 CON HN[25],J, D\$[25]

10 CLEAP 2 DISP 2 DISP $e$ DISP e DISP

28 DISP w

Program TRAHS1

30 DISP

khrezai"

40 DISP

$r c h$

50 DISP $\cdots$

rued"

60 DISP $Q$ DISP Q DISP MPress CO NT to continue"

79 PAUSE

8 REMOTE 711

9 CFLAG 1 e CFLAG 2

180 DIM C\$[2B], R\$[2056], B\$[20], A (1924)

110 INTEGER Z1

129 IOBUFFER AS

130 ON KEY* 1, "D ENTR" GOSUB 230

14 ON KEY* $2, " R$. CNT" GOSUE 640

159 와 KEY 3,"T.R.PL" GOSUB 860

160 ON KEY* 4,"FFT PL " GOSUB 110 a

179 DN KEY* 5, "SAVE" GOSUB 1309

189 ON KEY* 6,"TRANS2" GOSUB 179 D

199 ON KEY* 7, "COMBINE* GOSUB 20 99

200 OH KEY" 8, "TRANS3" GOSUB 256 a

205 CLEAR

210 KEY LABEL

229 GOTO 219

239 ! DATA ENTERY ROUTIHE*******

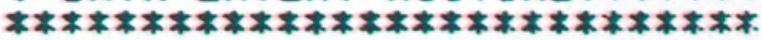
$* * * * *$

235 CLEAR

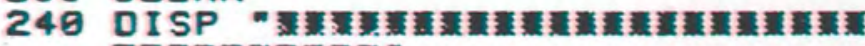

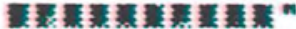

250 DISP = etter ce DISP " w

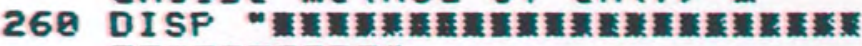

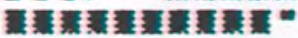

270 DISP DISP " Fi le ke dispe disf a

280 LINPUT * Analyzer , Cs

290 IF C\$="F" THEN CLEAR 2 GOTO 1569

309 IF $C \$={ }^{\circ} A "$ THEH CLEAR C GOSUB 90 무

310 IF $C \$=" A "$ THEN 1710

320 IF $C:=" K "$ THEN 350

339 CLEAR

348 COTD 230

350 DISP "NUMBER OF PTS. 2 INPU T N1 
360 LINFUT "A PLOT OF INPUT? ( $\%$ ' N) $\cdots, D=$

370 IF D $5={ }^{\circ} N$ - THEM 430

380 LINPUT "USING THE PLOTTER",D \$ 2 IF D\$="Y" THEN PLOTTER I

399 INPUT N2E PEN N2

400 SCALE $0, N 1,-1,1$

410 MOUE $20,-.9$ Q LABEL "\#FF PTS M, N1

429 AXES $100,1,9,0,1024,1,5$

430 FOF: $I=0$ TO $N 1-1$

440 DISP "ENTER POINT ", I 2 INP UT $Z 2$

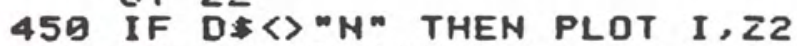

$460 \quad 22=(2 \wedge 15-1) * Z 2$

$470 \quad Z 1=Z 2$

480 OUTPUT A\$ USING "*,W"; Z1

490 NEXT I

509 FOR $J=I$ TO 1823

$51021=9$

520 OUTPUT A\$ USING "*,N"; $Z 1$

530 NEXT J

549 LINPUT "WANT TO SAUE THE DAT A? $(Y \text { OR. } N)^{\prime \prime, D}$

559 IF DS="Y" THEN GOSUB 1399

560 ! RETLRH OF ALL SEHDS*******. **************************** *

570 OUTPUT $711 ; " F R 1 R P Q "$

580 OUTPUT 711 USING "14A"; "WT M, 79999, $1024 \%$

590 TRANSFER FI TO 711 FHS

69日 CONTROL A\$1; 1 1024*2

610 DISP "DONE" 2 BEEP 6,1009

615 CLEAR

629 IF FLAG 2$\rangle$ THEN RETURN

630 GOTO 640

640 ! REMOTE CONTROL ROUTINE**** ***************************** *****

650 CLEFR

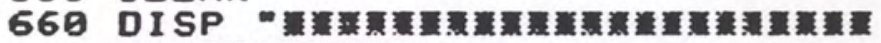

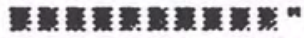

670 DISP " Four control codes $h$ ere"

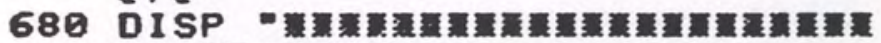

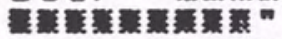

690 DISP क RTN $\gg$ return to Denu (1)

700 DISP " GO > send data to an lyzer

710 DISP - SAUE $\gg$ save time rec ord"

720 DISP " MRKR \> marker sub ro utine"

730 DISP - AUG $>$ Average wrt me an

735 DISP - SRQ $>$ service reques " recovery" 


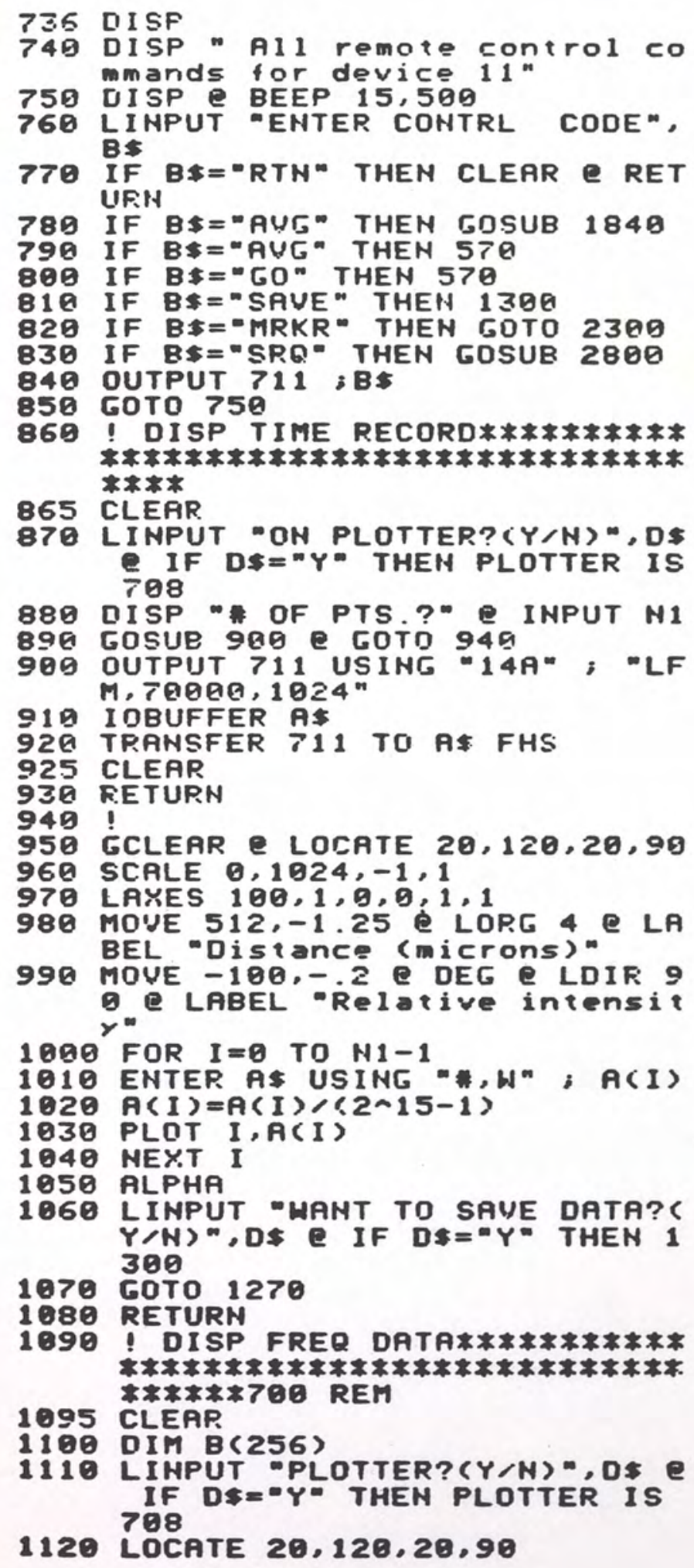

869 ! DISP TIME RECORD**********

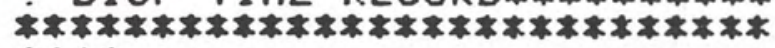
******

865 CLEAR.

870 LINPUT "ON PLOTTER? $(Y / N) \cdots, D \$$ IF DS="Y" THEH PLOTTER IS DISP " OF PTS.?"

1120 LoCate $20,120,20,90$ 


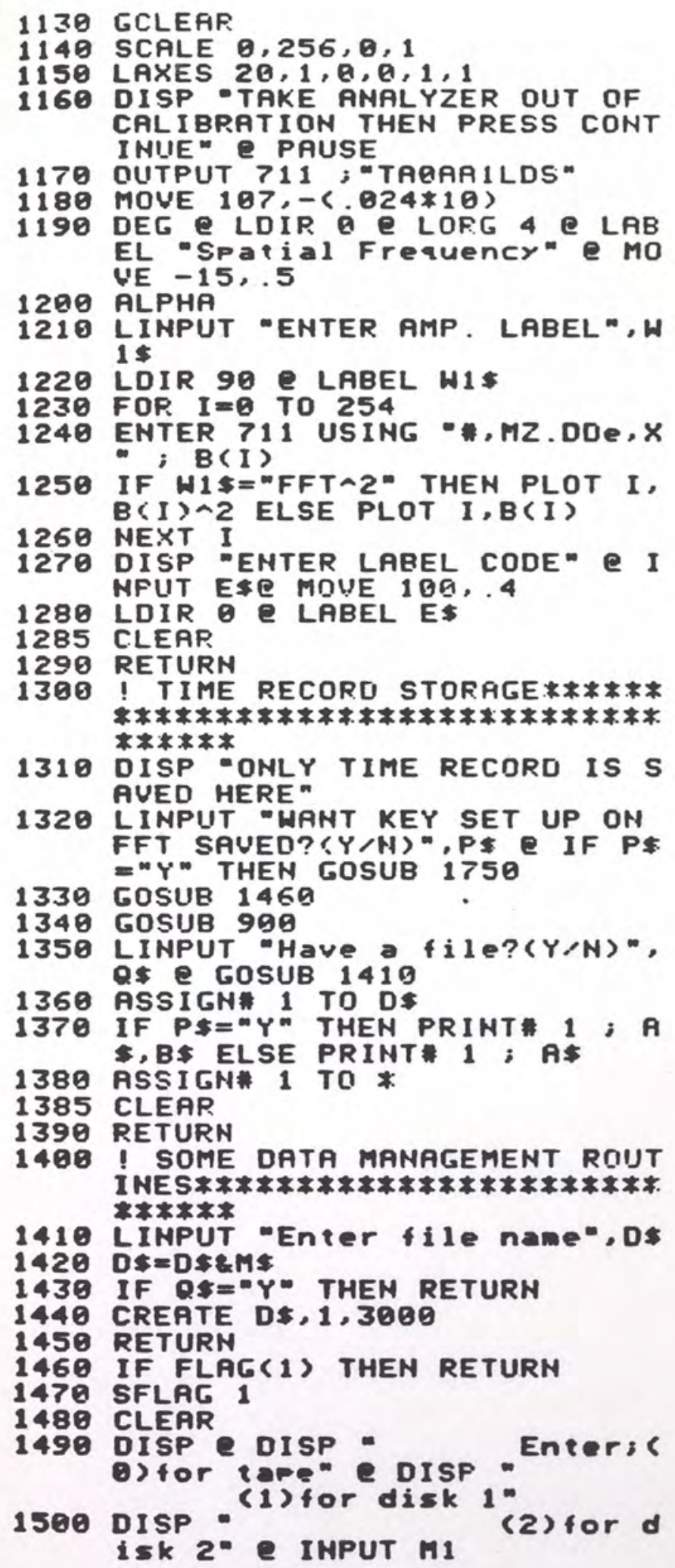

1310 DISP - ONLY TIME RECDRD IS S AUED HERE"

1320 LINPUT "WANT KEY SET UP ON FFT SAUED? $(Y / H) ", P$ \& IF $P$ * $=" Y "$ THEN GOSUB 1750

1330 GOSUB 1469

1340 GOSUB 990

1350 LINPUT "Have a file?(Y/N)", Q\$ 2 gosub 1410

1368 ASSIGNA 1 TO D\&

1370 IF P\$="Y" THEN PRINT\# $1 ; A$ \$, B\$ ELSE PRINT* $1 ; A \$$

1408 ! SOME DATA MANAGEMENT ROUT INES $* * * * * * * * * * * * * * * * * * * * * * *$. $* * * * * * *$

1410 LINPUT "Enter file name", D\$ 1420 D\$ $=D \$ 2 M \$$

1430 IF Q\$=-Y\% THEN RETURN

1440 CREATE D\$, 1,3080

1450 RETURM

1460 IF FLAG(1) THEN RETURN

1470 SFLAG 1

1480 CLEAR 1490 DISP DISP E DISP Enters

1500 DISP . (1) for disk 1" ISK 2" 2 INPUT MI 


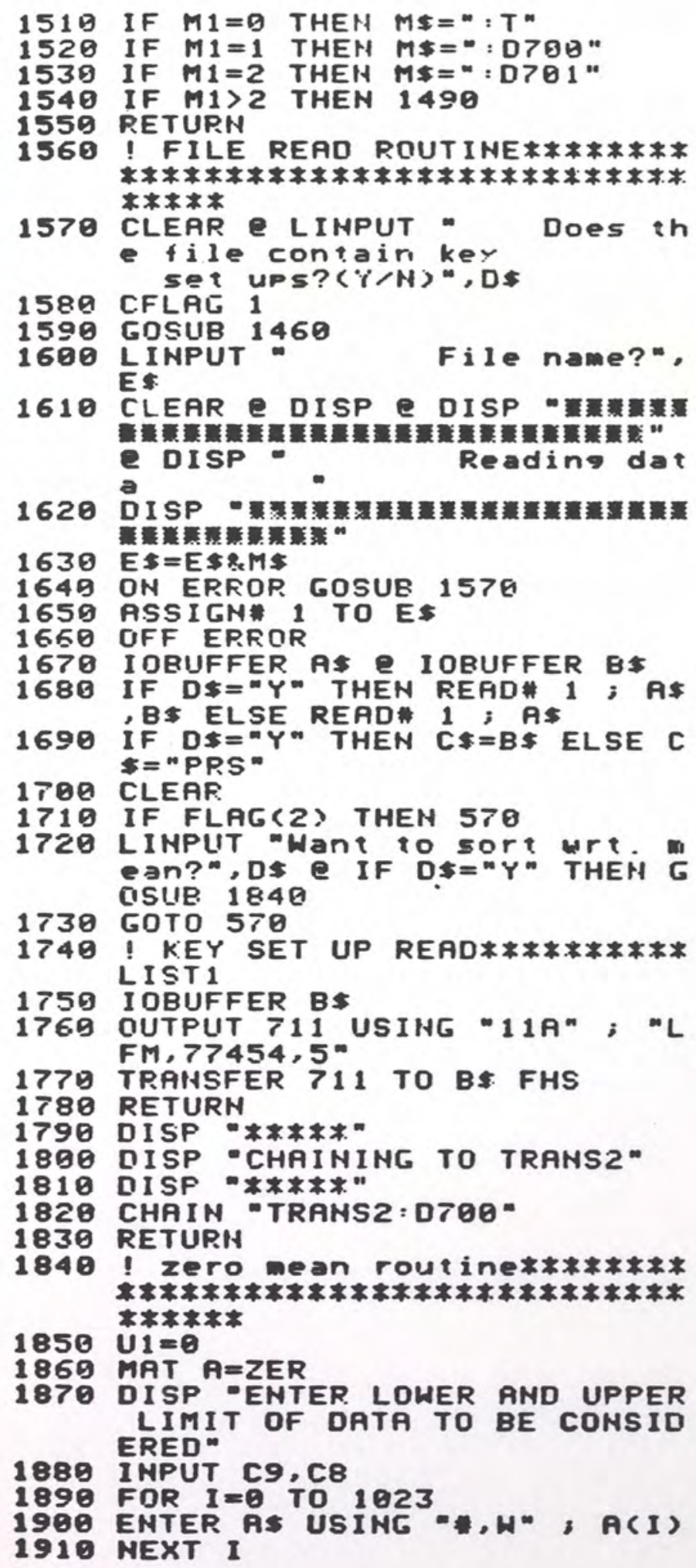




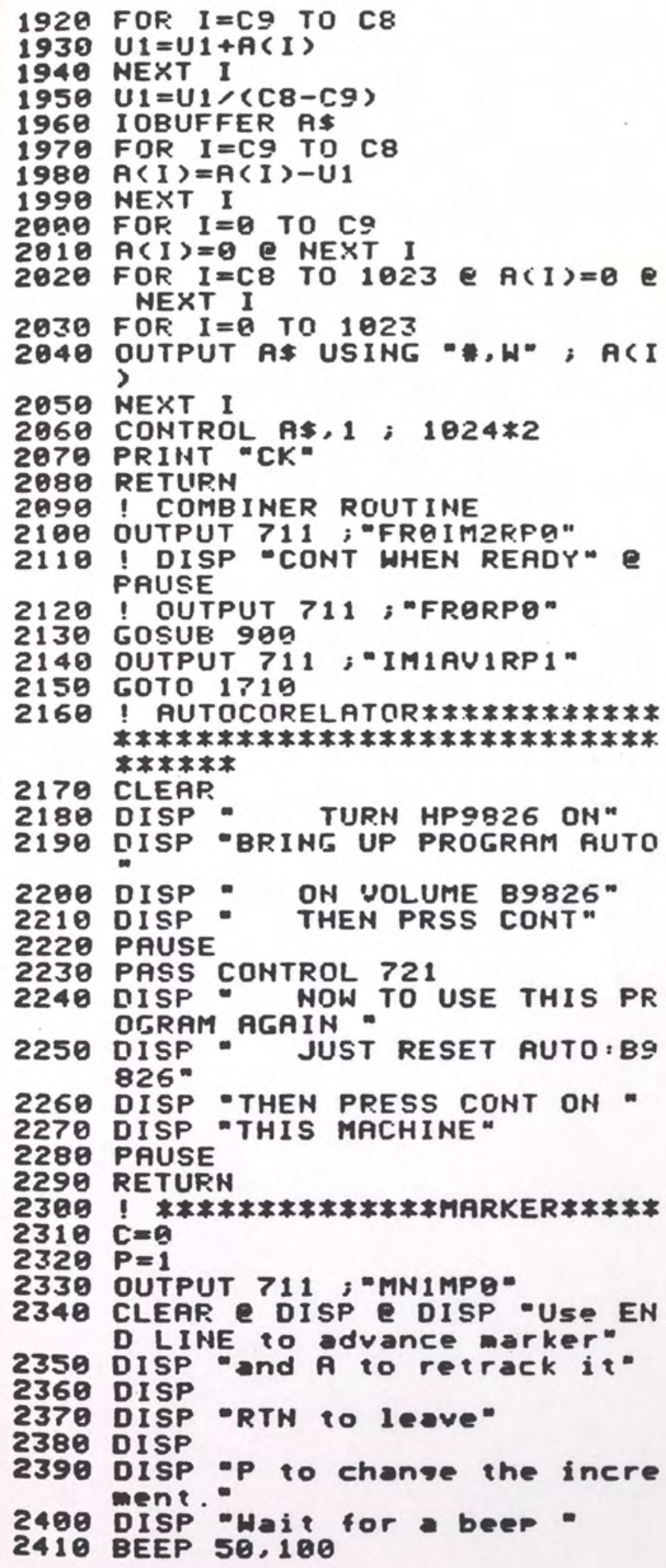




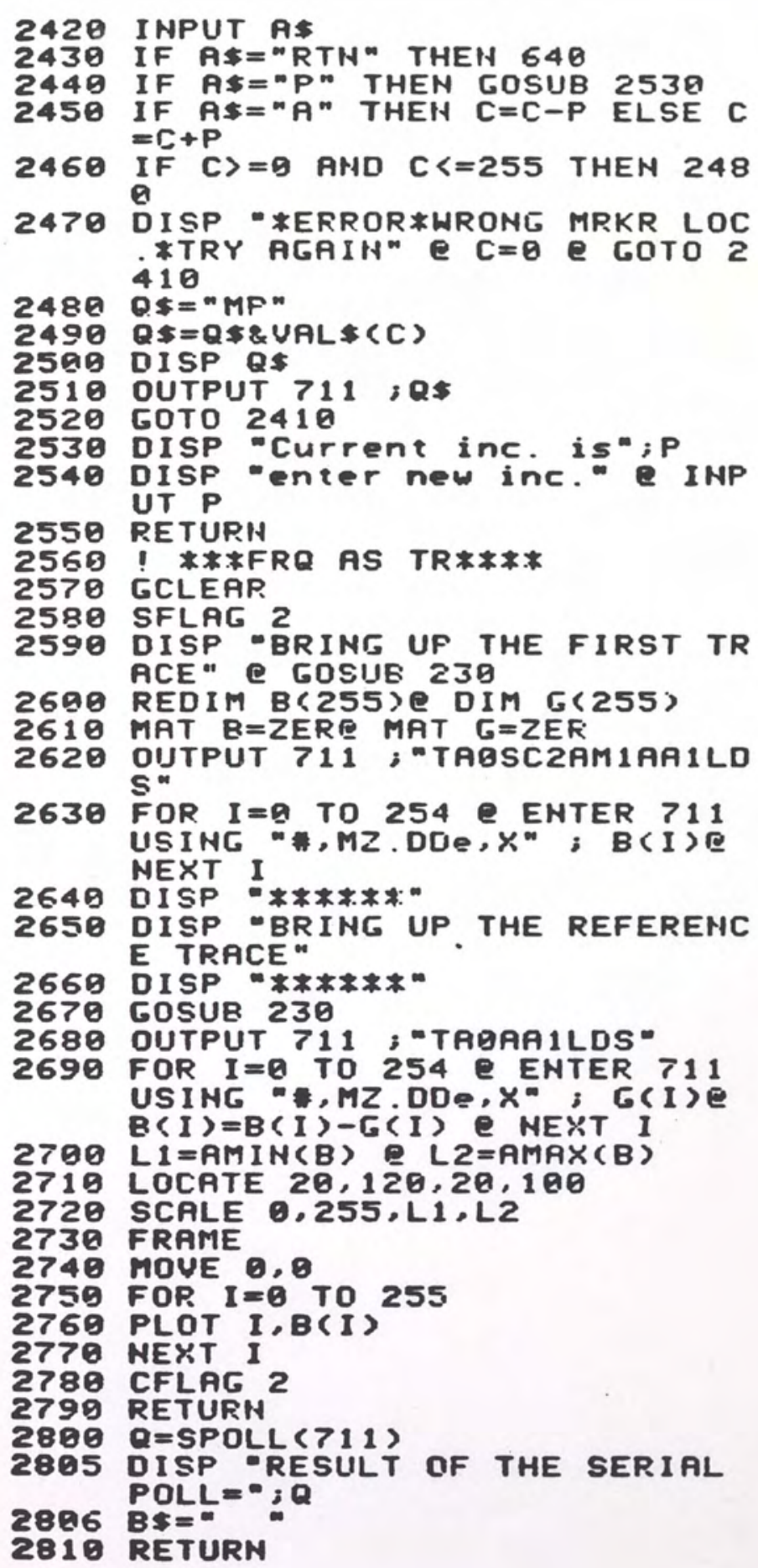




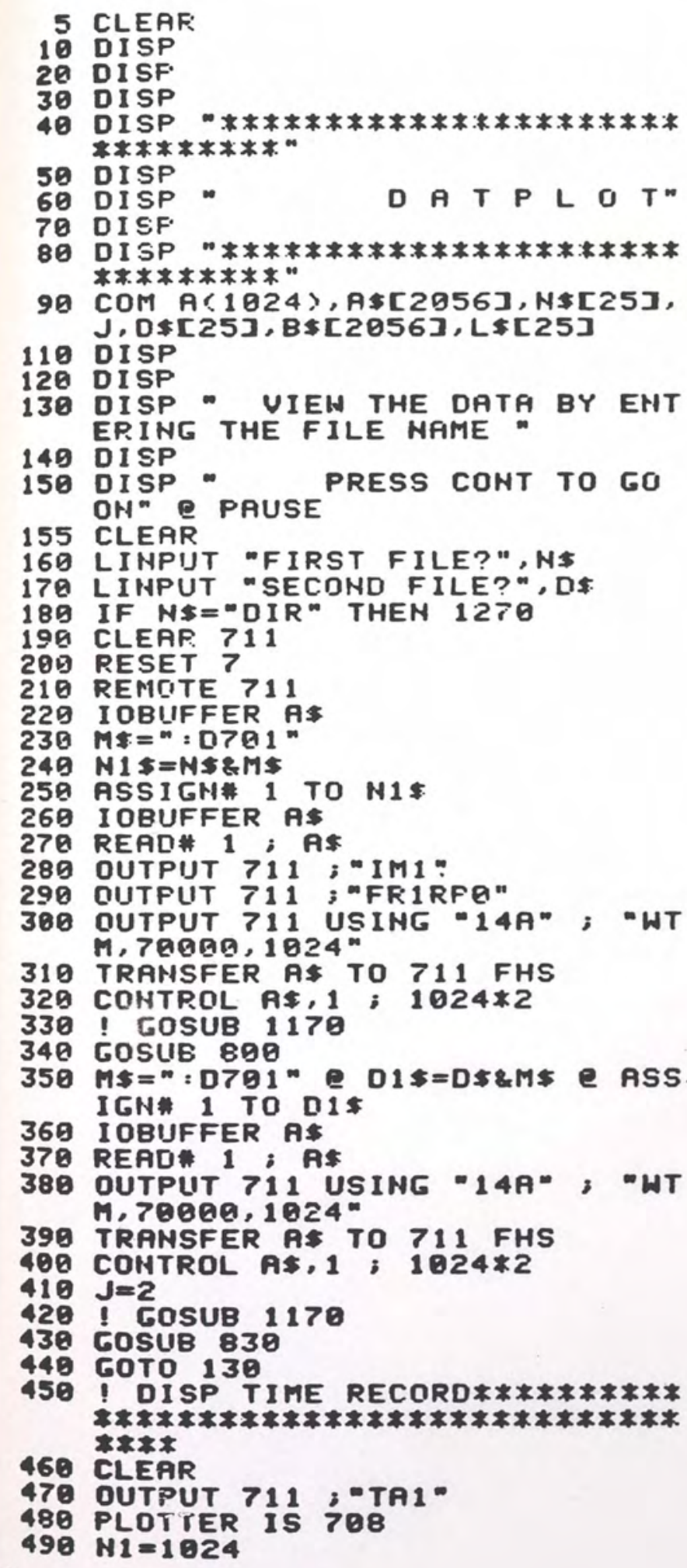




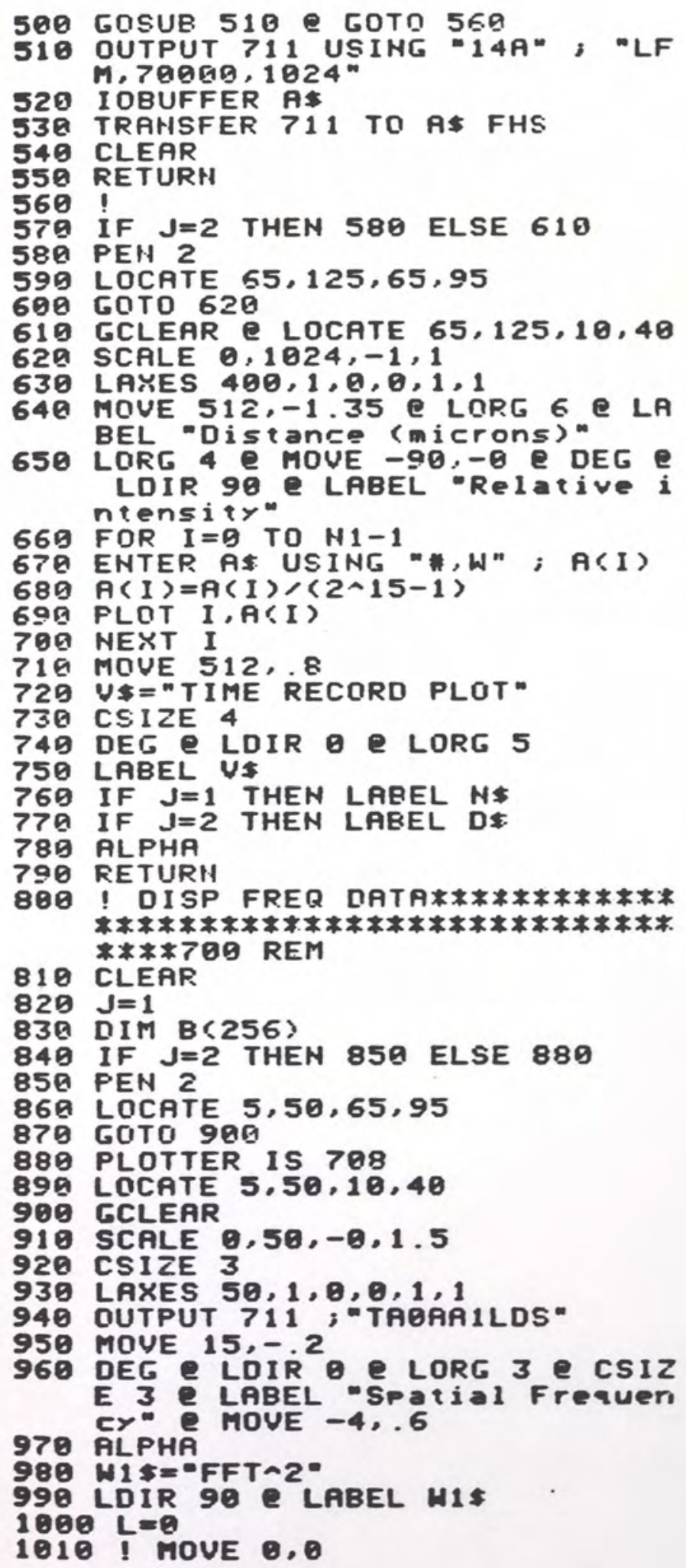




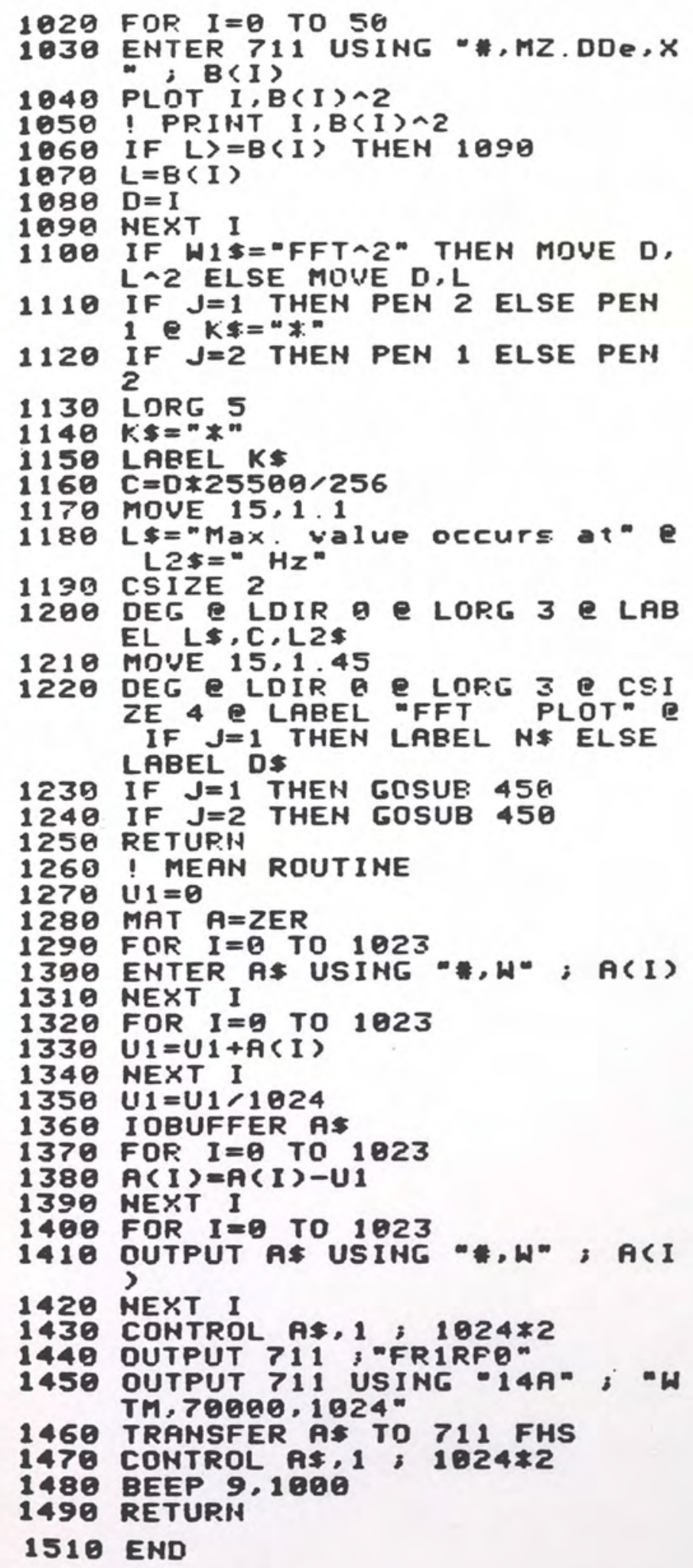


19 IISF

20 DISF

30 DISF

40 DISP

50 DISF

60 IISP **********

70 DISP

80 DISP

G

$\begin{array}{lllllll}P & L & \mathbf{O} & \mathbf{T} & \mathrm{T} & \mathrm{I} & \mathrm{N}\end{array}$

90 DISP

10 DISF

110 DISP “********************** ***********

120 COM $A(1024), A \$[2056], N \leqslant[25]$, $1, D \leq[25]$

130 DISP

140 DISF

150 DISP * UIEW THE DATA BY ENT ERING THE FILE NAME *

169 DISP

170 DISP " PRESS CONT TO GO ON" 2 PAUSE

189 CLERR.

199 LINPUT "FILE MAME?", $\mathrm{N} \$$

290 CLEAF 711

21 RESET 7

220 REMOTE 711

239 IOBUFFER A\$

$240 M \$=" D 701 \%$

$250 \mathrm{~N} \$=N \$ 2 \% \$$

260 ASSIGN\# 1 TO HF

279 IOBUFFER A\$

289 REAL* 1 ; $A *$

299 OUTPUT 711 ; "IMI"

300 DUTPUT 711 ; "FRIRPO"

310 DUTPUT 711 USING "14A"; "WT M, $70000,1024 \%$

320 TRANSFER A\$ TO 711 FHS

330 COHTROL A\$,1;1024*2

349 ! GOSUB 820

350 GOSUR 620

369 LINPUT "TIME RECORD ?", A*

370 IF $A \$=" Y *$ THEH 429 ELSE 390

38 DISP "DONE" 2 BEEP 6,1000

390 END

490 CLEAR

410 !

429 OUTPUT 711 ;"TA1 *

430 CLEAP

440 PLOTTER IS 708

450 OUTPUT 711 USING "14R"; "LF M. $79000,1024^{\circ}$

460 IOBUFFER A\$

470 TRANSFER 711 TO A\$ FHS

480 CLEAR

490 !

500 GCLEAR 2 LOCATE $20,120,20,90$

510 SCALE $0,1024,-1,1$

520 LAXES $100,1,0,0,1,1$ 


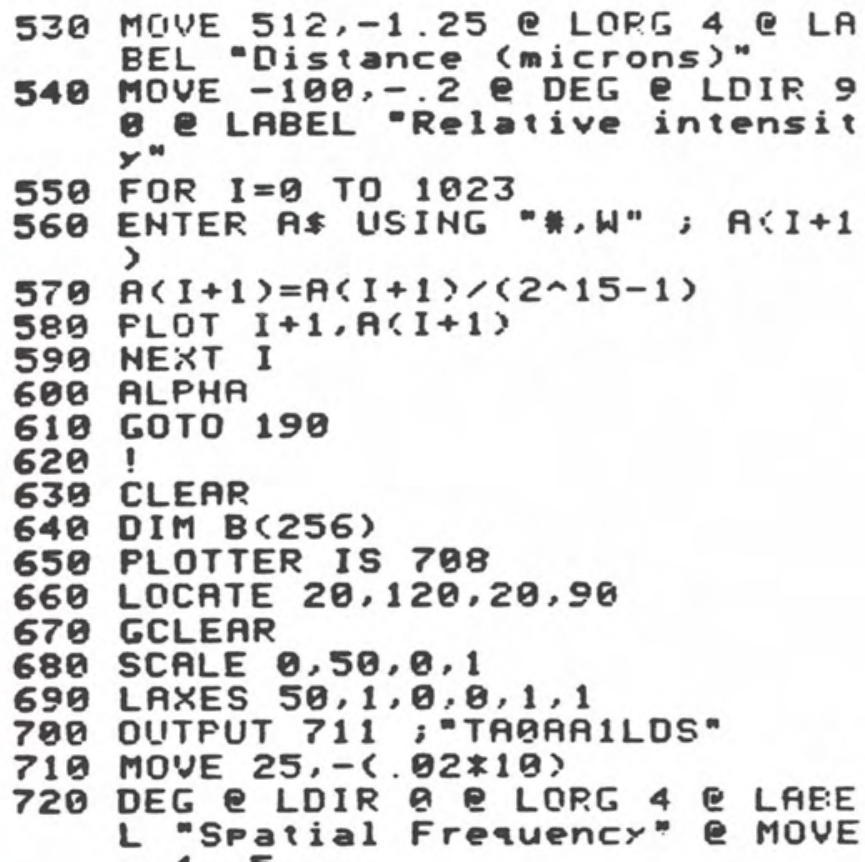
L Sparial Frequency" 2 MOVE 73日 ALPHÁ

740 W1\$="FFTA2"

759 LDIR 99 \& LABEL W1\$

$760 \mathrm{~L}=\mathrm{Q}$

770 FOR I=0 TO 59

780 ENTER 711 USING "\#,MZ.DDE, $X^{m}$ ; $B(I)$

790 IF W1\$="FFTA2" THEN PLOT I, B (I)A 2 ELSE PLOT I,B(I)

809 IF $L>=B(I)$ THEN 830 ELSE 810 $81 \mathrm{~L} L=B(I)$

$820 \mathrm{D}=1$

830 NEßT I

$840 \mathrm{D} 1=\mathrm{D} * 2550 \mathrm{~g} / 256$

850 IF HI\$="FFTA2" THEN MOUE $D, L$

860 LORG 5 Q 2 LABEL **

879 MOUE 30,55

880 DEG 2 LDIR 9 2 LORG 5 C LABE $L$ Max value is", D1 2 MOUE 4 $3, .55$ L LABEL ${ }^{\circ z}$

890 L\$ "FFF PLOT ${ }^{\circ}$ P MOUE $25, .9$

900 DEG 2 LDIR 9 e LORG 5 e CSIZ E 5 2 LABEL L\$

910 RETURH

920 !

$930 \quad$ U1 $1=0$

940 MAT $A=Z E R$

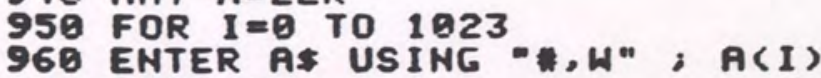

970 NEXT I

980 FOR $I=0$ TO 1023

$990 U 1=U 1+A(I)$

1000 NEXT I 


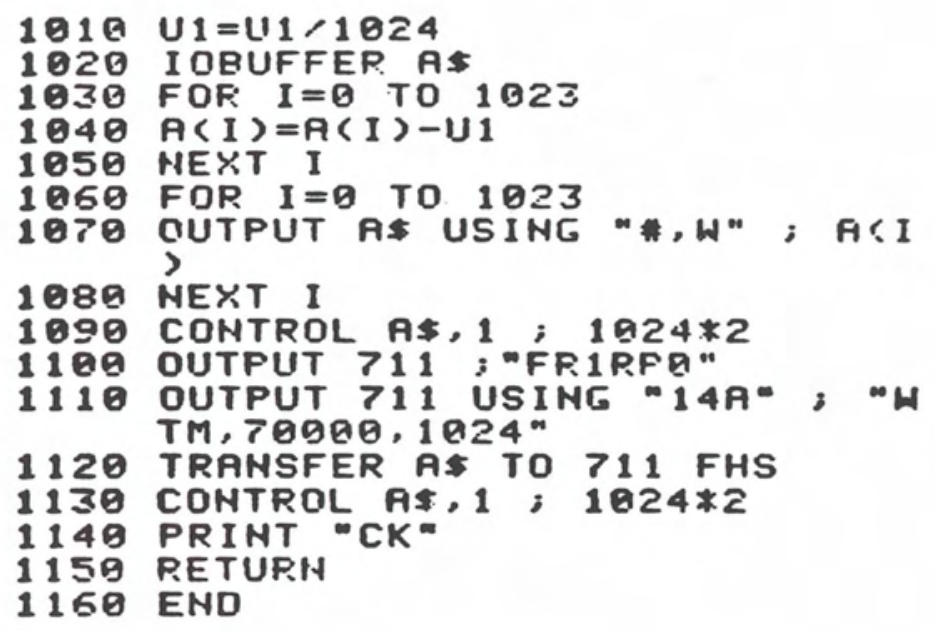




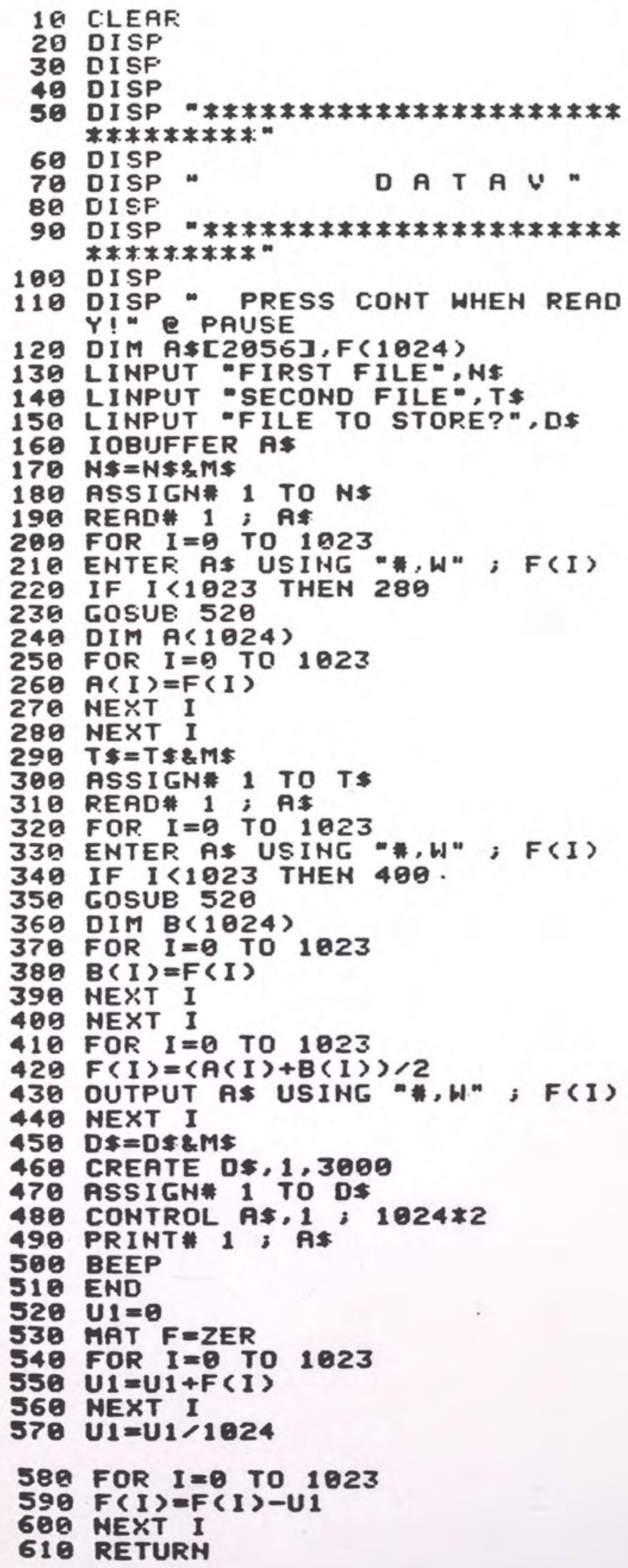




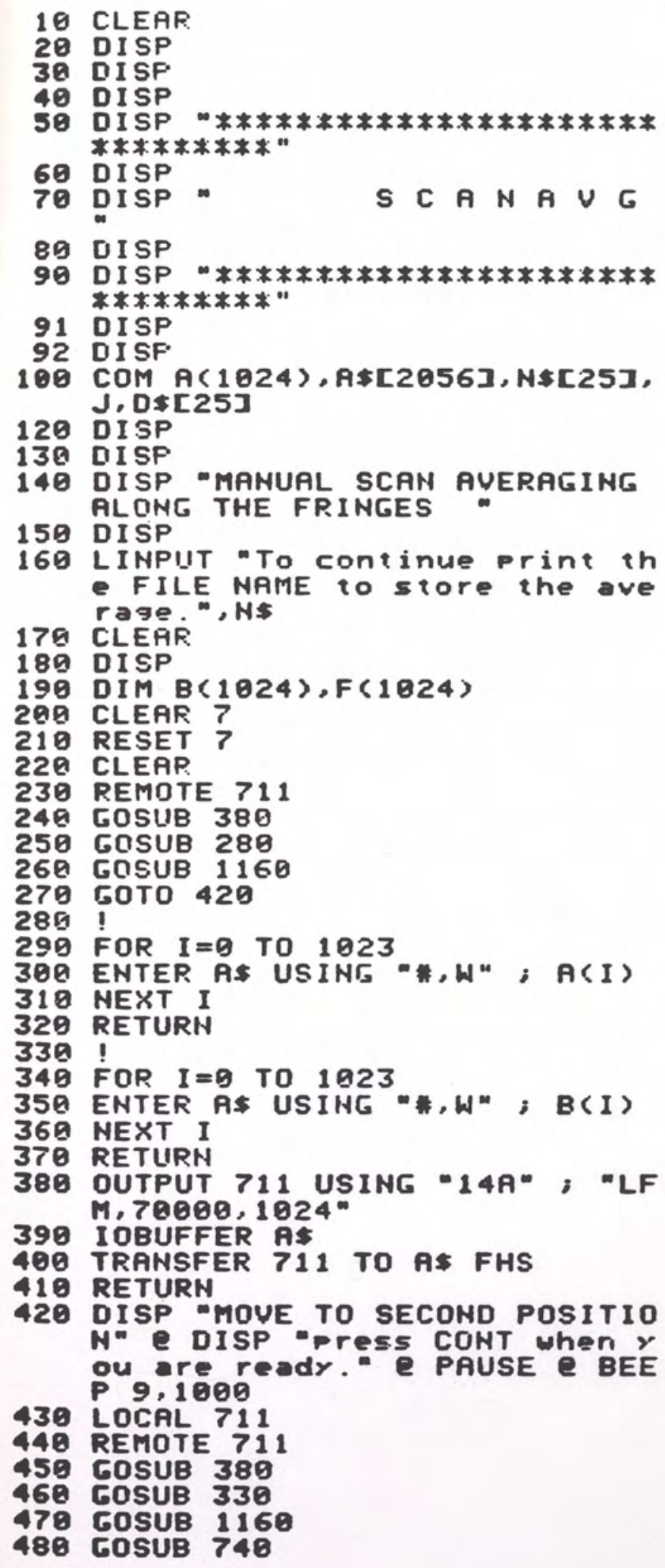




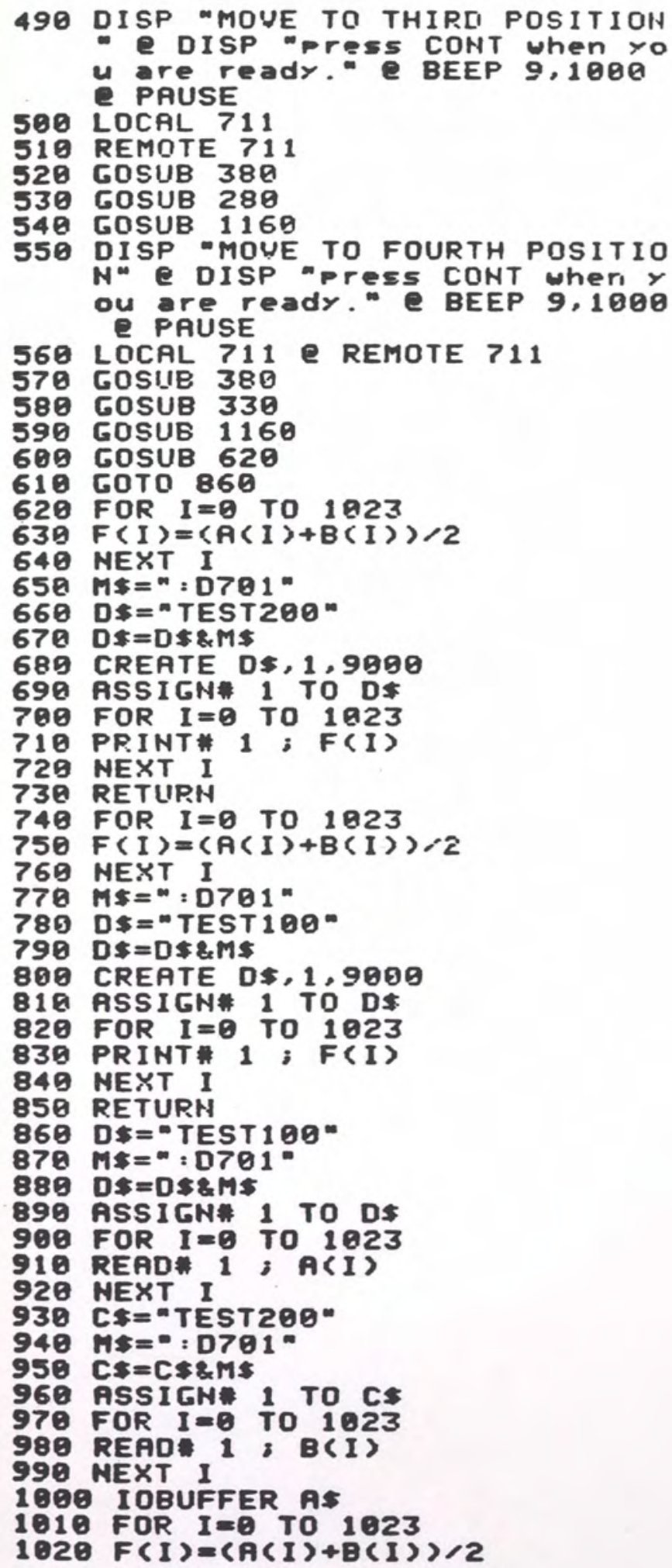



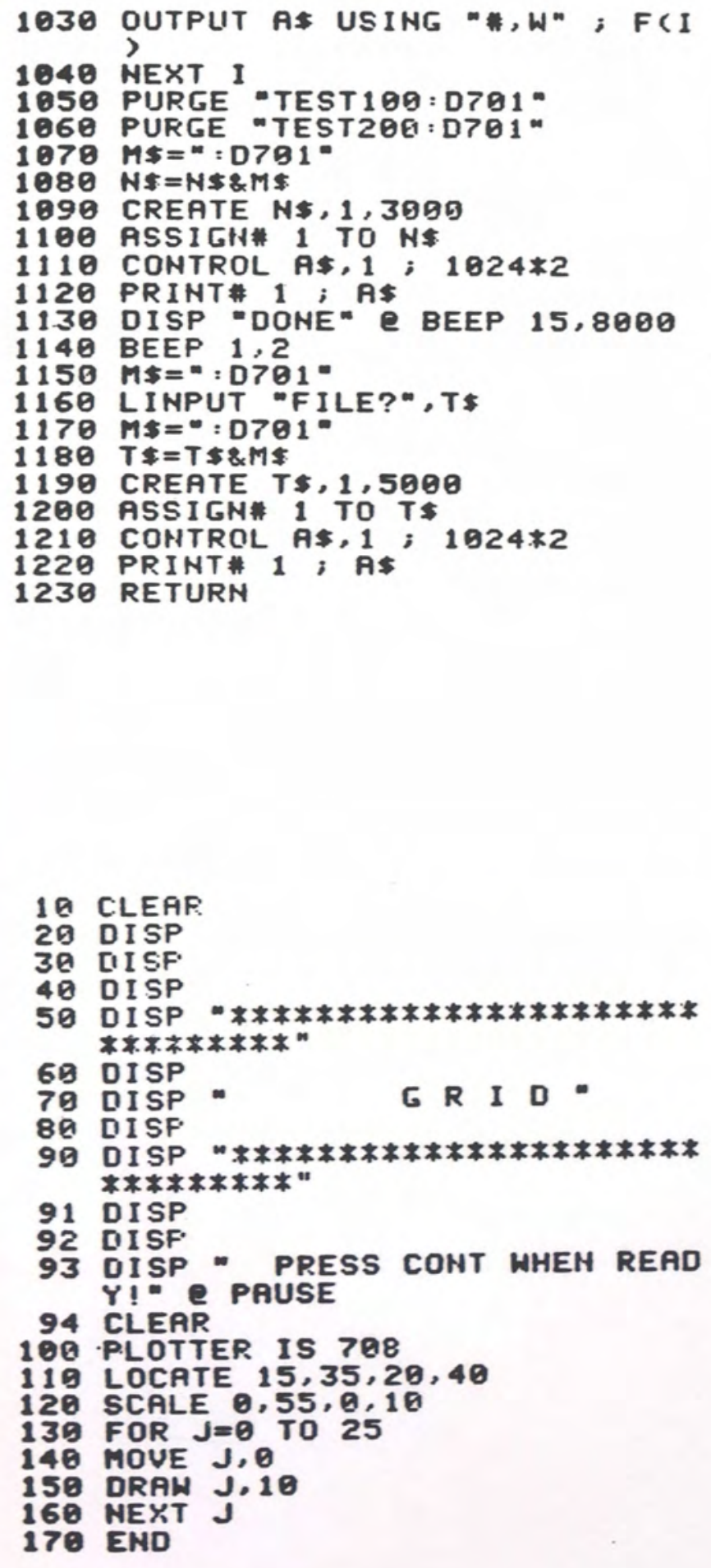


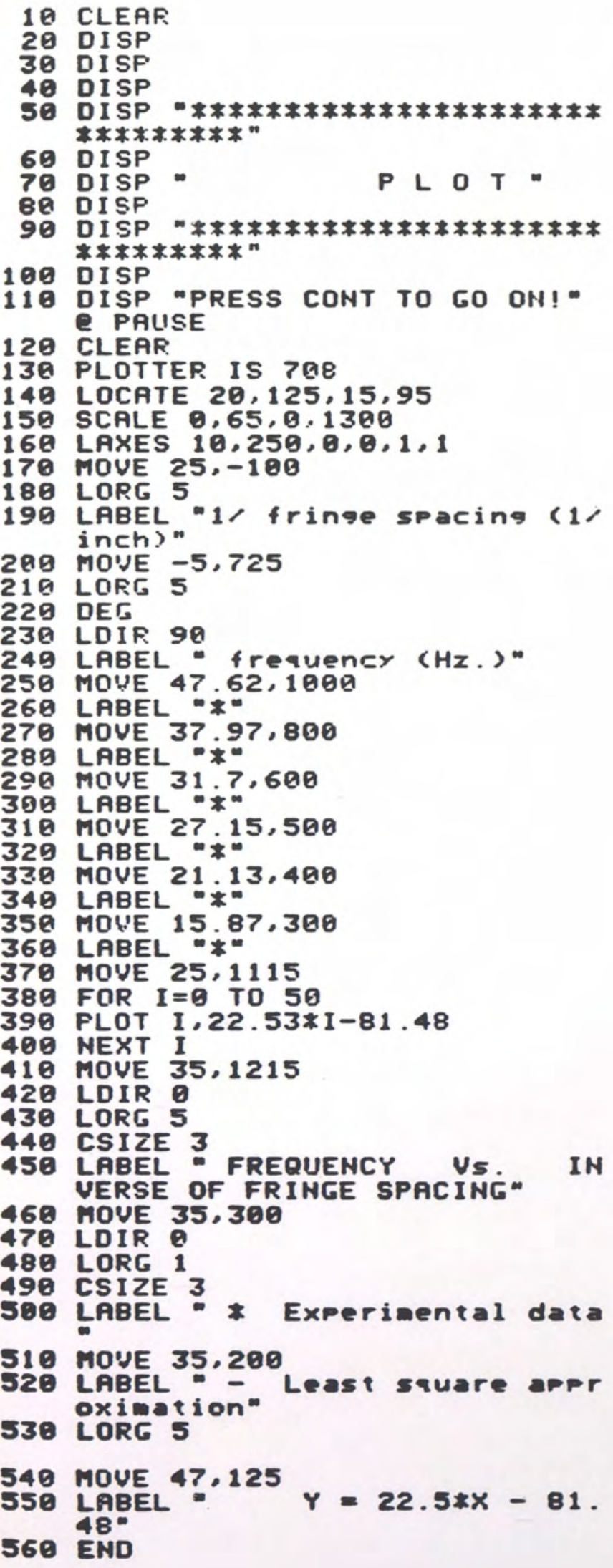




\section{REFERENCES}

1. Byars, E.F., and Snyder, R.D. Engineering Mechanics of Deformable Bodies. Pittsburgh: International Textbook Company, 1963.

2. Dally, J.W., and Riley, W. Experimental Stress Analysis. New York: McGraw-Hill Book Company, 1978.

3. Dove, R.C., and Adams, P.H. Experimental Stress Analysis and Motion Measurements. Cincinnati: Charles E. Merril Books, Inc., 1964.

4. Morse, S.; Duerilli, A.J.; and Sciammarella, C.A. "Geometry of Moire Fringes in Strain Analysis." Journal Engineering 86, EM4 (August 1960): 105-126.

5. Collier, R.J.; Burchhardt, C.B.; and Lin, L.H. Optical Holography. New York: Academic Press, 1971.

6. Vest, C.M. Holographic Interferometry. Chicago: John Wiley and Sons, 1979.

7. Holman, J.P. Experimental Methods for Engineers. New York: McGraw-Hill Book Company, 1984.

8. Gabor, D. "Microscopy by Reconstructed Wavefronts." Proceedings of the Royal Society Al97 (June 1949): 454-487.

9. Leith, E.N., and Upatnieks, J. "Reconstructed Wavefronts and Communication Theory." Journal of the Optical Society of America 52 (June 1962): 1123.

10. Leith, E.N., and Upatnieks, J. "Wavefront Reconstruction with Continuous-tone Objects." Journal of the Optical Society of America 53 (August 1963): 1377.

11. Horman, M.H. "An Application of Wavefront Reconstruction to Interferometry." Application of Optics 4 (January 1965): 333-336.

12. Powell, R.L., and Stetson, K.A. "Interferometric Analysis by Wavefront Reconstruction." Journal of the Optical Society of America 55 (August 1965): 1593-1598. 
13. Burch, J.M. "The Application of Lasers in Production Engineering." Production Engineering 44 (June 1965): 431-442.

14. Collier, R.J.; Doherty, E.T.; and Pennington, K.S. "Application of Moire Techniques." Applied Physics 7 (July 1965): 223225 .

15. Steson, K.A., and Powell, R.L. "Hologram Interferometry." Journal of the Optical Society of America 55 (September 1965): 1570A.

16. Haines, K.A., and Hildebrand, B.P. "Surface-Deformation Measurement Using the Wavefront Reconstruction Technique." Applied Optics 5 (December 1966): 595-602.

17. Brooks, R.E.; Heflinger, L.0.; and Wuerker, R.F. "Interferometry with a Holographically Reconstructed Comparison Beam." Applied Physics 7 (June 1965): 248-249.

18. Heflinger, L.O.; Wuerker, R.F.; and Brooks, R.E. "Holographic Interferometry." Joumal of Applied Physics 37 (August 1966): 642-649.

19. Gabor, D.; Stroke, G.W.; Restrick, R.; Funkhoouser, A.; and Brumm, D. "Optical Image Synthesis (Complex Amplitude Addition and Subtraction) by Holographic Fourier Transformation." Applied Physics 18 (September 1965): 116-118.

20. Aleksandrov, E.B., and Bonch-Bruevich, A.M. "Investigation of Surface Strains by the Hologram Technique." Soviet Physics 12 (June 1967): 258-265.

21. Ennos, A.E. "Measurements of In-plane Surface Strain by Hologram Interferometry." Journal Scientific Instruments Series 11 (March 1968): 731-746.

22. Wilson, A.D. "In-plane Displacement of a Stressed Membrane with a Hole Measured by Holographic Interferometry." Applied Optics 10 (July 1971): 908-912.

23. Barnet, N.E. "Vibration Analysis by Holography." Journal of the Optical Society of America 57 (April 1967): 1406A.

24. Molin, N.E., and Stetson, K.A. "Measuring Combination Mode Vibiation Patterns by Hologram Interferometry." Journal Phy:3ics and Scientific Instrumentation 2 (May 1969): 609-612. 
25. Stetson, K.A. "Effects of Beam Modulation on Fringe Loci and Localization in Time-Average Hologram Interferometry." Journal of the Optical Society of America 60 (March 1970): 1378-1388.

26. Wilson, A.D., and Strope, D.H. "Time-Average Holographic Interferometry of a Circular Plate Vibrating Simultaneously in Two Rationally Related Modes." Journal of the Optical Society of America 60 (June 1970): 1162-1165.

27. Tiziani, H.J. "A Study of the Use of Laser Speckle to Measure Small Tilts of Optically Rough Surfaces Accurately." Optics Communications 5 (September 1972): 271-276.

28. Archbold, E., and Ennos, A.E. "Displacement Measurement from Double-Exposure Laser Photography." Optica Acta 19 (August 1972) : 253-271.

29. Archbold, E.; Burch, J.M.; and Ennos, A.E. "Recording of In-Plane Surface Displacement by Double-Exposure Speckle Photography." Optica Acta 17 (July 1970): 883-898.

30. Tiziani, H.J. "Analysis of Mechanical Oscillations by Speckling." Applications of Optics 11 (March 1972): 2911-2917.

31. Kopf, U. "A Coherent Optical Method for Measurements of Mechanical Oscillation." Optik 33 (September 1971): 517.

32. Stetson, K.A. "Problems of Defocusing in Speckle Photography: Its Connection to Hologram Interferometry and Its Solution." Journal of the Optical Society of America 66 (January 1976): 1267-1271.

33. Adams, F.D., and Maddux, G.E. "Synthesis of Holographic Interferometry and Specile Photography to Measure 3-D Displacements." Applied Optics 13 (January 1974): 219.

34. Boone, P.M. "Use of Reflection Holograms in Holographic Interferometry and Speckle Correlation for Measurement of Surface Displacement." Optica Acta 22 (February 1975): 579-590.

35. Hudson, R.R., and Setopoulos, D.D. "Speckle Interferometric Method for the Determination of Time-Dependent Displacements and Strains." Journal of Applied Phys1cs 11 (May 1975): 126-129.

36. Maddux, G.E.; Moorman, S.L.; and Corwin, R.R. Programmable Data Retrieval Systems for In-Plane Displacements from Speckle Photographs. New York: U.S.A.F. Technical Report \#AFFDL-TM-78-109-FBE [1978]. 
37. Ineichen, B.I.; Eglin, P.; and Dandliker, R. Hybrid Optical and Electronic Image Processing for Strain Measurements by Speckle Photography. New York: International Computer Optics Society, 1978.

38. Kaufmann, G.H.; Ennos, A.E.; Gale, B.; and Pugh, D.J. "An Electro-Optical Read-out System for Analysis of Speckle Photographs." Journal Physics and Scientific Instrumentation 13 (March 1980): 579-584.

39. Kaufmann, G.H. "Digital Analysis of Speckle Photography Fringes Processing of Experimental Data." Applied Optics 21 (June 1982): 3411-3412.

40. Yamaguchi, I. "A Laser Speckle Strain Gage." Journal Physics and Scientific Instrumentation 14 (July 1981): 1270-1273.

41. Yamaguchi, I. "Fringe Formation in Speckle Photography." Journal of the Optical Society of America 1 (January 1984): 81-86.

42. Muramatsu, A., and Lunazzi, M. "Advantages of a Derivative Technique in Performing Speckle Correlation." Society of Applied Optics 23 (July 1984): 3038-3039.

43. Tippens, P.E. Applied Physics. New York: McGraw-H1ll Book Company, 1973.

44. Timoshenko, S.P., and Gere, J.M. Theory of Elastic Stability. New York: McGraw-Hill Book Company, 1961.

45. Sheikhrezai, R.S. "A Non-Contact Technique for Direct Strain Measurement Using Laser Speckle Spectral Density." Master's Thesis, University of Central Florida, Orlando, 1984. 